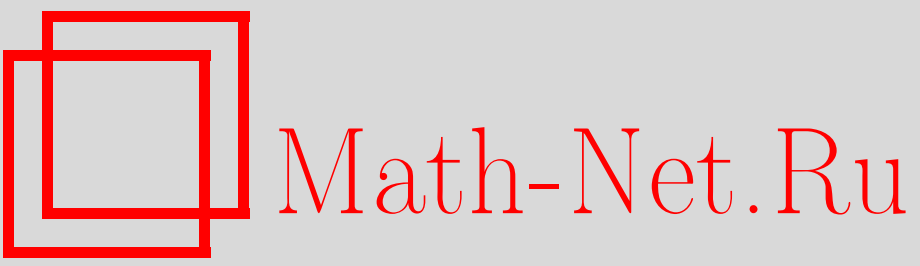

Е. И. Кайкина, П. И. Наумкин, И. А. Шишмарев, Задача Коши для уравнения типа Соболева со степенной нелинейностью, Изв. РАН. Сер. матем., 2005, том 69, выпуск 1, 61-114

DOI: https://doi.org/10.4213/im624

Использование Общероссийского математического портала Math-Net.Ru подразумевает, что вы прочитали и согласны с пользовательским соглашением

http: //www . mathnet.ru/rus/agreement

Параметры загрузки:

IP : 35.173 .219 .149

26 апреля 2023 г., 07:46:17 


\title{
Задача Коши для уравнения типа Соболева со степенной нелинейностью
}

\begin{abstract}
Исследовано асимптотическое поведение при больших временах решений задачи Коши для нелинейного уравнения типа Соболева с диссипацией. Используемый подход в случае малых начальных данных основан на детальном изучении функции Грина линейной задачи и применении метода сжимающих отображений. Рассмотрен также случай больших начальных данных. В суперкритическом случае асимптотика имеет квазилинейный характер. Асимптотическое поведение решений в критическом случае отличается от поведения решений соответствующего линейного уравнения логарифмической поправкой. В субкритическом случае доказано, что если начальные данные имеют ненулевую общую массу, то главный член асимптотики решения при больших временах представляется автомодельным решением.

Библиография: 51 наименование.
\end{abstract}

\section{§1. Введение}

Настоящая работа посвящена изучению задачи Коши для уравнения типа Coболева

$$
\begin{gathered}
\partial_{t}(u-\Delta u)-\alpha \Delta u=\lambda|u|^{\sigma} u, \quad x \in \mathbb{R}^{n}, \quad t>0, \\
u(0, x)=u_{0}(x), \quad x \in \mathbb{R}^{n},
\end{gathered}
$$

где $\alpha>0, \sigma>0, \lambda \in \mathbb{R}$. Уравнениями типа Соболева описываются многие физические процессы (см. работы [41], [9], [13], [31], [32], [37]).

В последнее время большое внимание уделялось изучению уравнений типа Соболева. Теория полугрупп применялась к общей теории сингулярных уравнений типа Соболева в работе [40]. Уравнения типа Соболева со знакопеременным или необратимым оператором при старшей производной по времени изучались в [14]. Вырожденные уравнения типа Соболева исследовались с абстрактной точки зрения в книге [17]. В книге [12] получено много важных результатов для систем уравнений, не разрешенных относительно старшей производной по времени. В ряде случаев уравнения типа Соболева называются также псевдопараболическими уравнениями. Локальная разрешимость уравнений типа Соболева рассмотрена в книге [10]. Уравнения типа Соболева с двумя нелинейностями рассматривались в [43]. Псевдопараболические уравнения с монотонной нелинейностью изучались в работе [48]. В работах [29], [30] доказано глобальное сушествование ограниченных решений и обнаружен эффект разрушения решений уравнений типа Соболева. Исследования разрушения решений различных классов нелинейных параболических уравнений проведены в работах [18], [33], [36]. Асимптотическое поведение при 
больших временах решения задачи Коши для уравнения типа Соболева с нелинейностью конвективного типа изучалось в работах [1]-[4], [7], [26], [27], [34], [35], [38], [49], [50].

Насколько нам известно, асимптотическое поведение при больших временах решений задачи Коши для нелинейного уравнения типа Соболева с неконвективной нелинейностью степенного вида (1) не было изучено до сих пор. В настоящей работе мы восполним этот пробел. Асимптотика при больших временах решений задачи (1) оказывается различной в следующих трех случаях. В суперкритическом случае $\sigma>\frac{2}{n}$ нелинейность является асимптотически слабой и не влияет существенно на асимптотическую форму решений. В критическом случае $\sigma=\frac{2}{n}$ нелинейность определяет логарифмическую поправку в главном члене асимптотики по сравнению с соответствующим линейным уравнением. Наконец, в субкритическом случае $\sigma \in\left(0, \frac{2}{n}\right)$ асимптотика при больших временах задается специальньм автомодельным решением. Отметим, что подобное поведение было ранее обнаружено для нелинейного уравнения теплопроводности

$$
\partial_{t} u-\Delta u+u^{1+\sigma}=0
$$

где $\sigma>0$ (см. работу [25] в суперкритическом случае $\sigma>\frac{2}{n},[11]$ в критическом случае $\sigma=\frac{2}{n}$ и работы [15], [16], [19], [28] в субкритическом случае $\left.\sigma \in\left(0, \frac{2}{n}\right)\right)$.

Через $\mathscr{F} \varphi$ или $\widehat{\varphi}$ будем обозначать преобразование Фурье функции $\varphi$, определяемое формулой

$$
\widehat{\varphi}(\xi)=(2 \pi)^{-\frac{n}{2}} \int_{\mathbb{R}^{n}} e^{-i x \xi} \varphi(x) d x
$$

тогда

$$
\mathscr{F}^{-1} \varphi(x)=(2 \pi)^{-\frac{n}{2}} \int_{\mathbb{R}^{n}} e^{i x \xi} \varphi(x) d x
$$

задает обратное преобразование Фурье функции $\varphi$. Через $\mathbf{C}(\mathbf{I} ; \mathbf{B})$ обозначим пространство непрерывных функций, отображающих интервал времени I в банахово пространство В. Пространство Лебега обозначаем, как обычно, через $\mathbf{L}^{p}$, $1 \leqslant p \leqslant \infty$. Определим также весовое пространство Лебега $\mathbf{L}^{p, a}$ следующим образом:

$$
\mathbf{L}^{p, a}=\left\{\varphi \in \mathbf{L}^{p} ;\|\varphi\|_{\mathbf{L}}{ }^{p, a}=\left\|\langle x\rangle^{a} \varphi\right\|_{\mathbf{L}^{p}}<\infty\right\},
$$

где $\langle x\rangle=\sqrt{1+|x|^{2}}$ - японские скобки, $a \geqslant 0,|x|=\sqrt{x_{1}^{2}+x_{2}^{2}+\cdots+x_{n}^{2}}$. Весовое пространство Соболева определим как

$$
\mathbf{W}_{p}^{k, a}=\left\{\varphi \in \mathbf{L}^{p} ;\|\varphi\|_{\mathbf{W}_{p}^{k, a}}=\sum_{j=0}^{k}\left\|\partial^{j} \varphi\right\|_{\mathbf{L}^{p, a}}<\infty\right\}
$$

где $k \geqslant 0, a \geqslant 0,1 \leqslant p \leqslant \infty$. В частности, при $p=2$ обозначим также $\mathbf{H}^{k, a}=\mathbf{W}_{2}^{k, a}$ и $\mathbf{W}_{p}^{k}=\mathbf{W}_{p}^{k, 0}$. Норму обычного пространства Соболева $\mathbf{H}^{k}=\mathbf{H}^{k, 0}$ зададим следуюшим образом: $\|\varphi\|_{\mathbf{H}^{k}}^{2}=\sum_{j=0}^{k}\left\|\partial^{j} \varphi\right\|_{\mathbf{L}^{2}}^{2}$.

В $\S 2$ мы получим предварительные оценки для линейной задачи Коши, соответствующей (1), и установим локальную во времени разрешимость нелинейной задачи (1). Затем в $\S 3-5$ мы изучим асимптотическое поведение при больших временах 
решений задачи Коши для нелинейного уравнения типа Соболева (1) в суперкритическом, критическом и субкритическом случаях соответственно.

\section{§2. Предварительные результаты}

2.1. Оператор Грина. Рассмотрим линейную задачу Коши

$$
\begin{gathered}
\partial_{t}(u-\Delta u)-\alpha \Delta u=f(t, x), \quad x \in \mathbb{R}^{n}, \quad t>0, \\
u(0, x)=u_{0}(x), \quad x \in \mathbb{R}^{n} .
\end{gathered}
$$

Используя преобразование Фурье и принцип Дюамеля, перепишем задачу (2) в виде интегрального уравнения

$$
u(t)=\mathscr{G}(t) u_{0}+\int_{0}^{t} \mathscr{G}(t-\tau) \mathscr{B} f(\tau) d \tau,
$$

или, более подробно,

$$
\begin{aligned}
u(t, x)= & \int_{\mathbb{R}^{n}} G(t, x-y) u_{0}(y) d y \\
& +\int_{0}^{t} d \tau \int_{\mathbb{R}^{n}} H(t-\tau, x-y) f(\tau, y) d y
\end{aligned}
$$

где оператор Грина $\mathscr{G}(t)$ определен с помощью обратного преобразования Фурье

$$
\mathscr{G}(t) \varphi=e^{-\alpha t} \overline{\mathscr{F}}_{\xi \rightarrow x} e^{\frac{\alpha t}{1+|\xi|^{2}}} \widehat{\varphi}(\xi)
$$

и оператор

$$
\mathscr{B} \varphi=(2 \pi)^{-\frac{n}{2}} \int_{\mathbb{R}^{n}} B(x-y) \varphi(y) d y
$$

задан ядром Бесселя-Макдональда (см. [44])

$$
B(x)=(2 \pi)^{-\frac{n}{2}} \int_{\mathbb{R}^{n}} e^{i \xi x}\left(1+|\xi|^{2}\right)^{-1} d \xi=|x|^{1-\frac{n}{2}} K_{\frac{n}{2}-1}(|x|),
$$

где

$$
K_{\nu}(|x|)=K_{-\nu}(|x|)=2^{-\nu-1}|x|^{\nu} \int_{0}^{\infty} \xi^{-\nu-1} e^{-\xi-\frac{|x|^{2}}{4 \xi}} d \xi
$$

- функция Макдональда (либо модифицированная функция Бесселя) порядка $\nu \in \mathbb{R}($ см. [8]). Пользуясь разложением в ряд Тэйлора

$$
e^{\frac{\alpha t}{1+|\xi|^{2}}}=\sum_{k=0}^{\infty} \frac{\alpha^{k} t^{k}}{k !}\left(1+|\xi|^{2}\right)^{-k}
$$

мы можем представить оператор Грина в виде

$$
\mathscr{G}(t) \varphi=e^{-\alpha t} \sum_{k=0}^{\infty} \frac{\alpha^{k} t^{k}}{k !} \mathscr{B}^{k} \varphi
$$


где $\mathscr{B}^{0}=1$ и

$$
\mathscr{B}^{k} \varphi=(2 \pi)^{-\frac{n}{2}} \int_{\mathbb{R}^{n}} B_{k}(x-y) \varphi(y) d y
$$

с ядром

$$
\begin{aligned}
B_{k}(x) & =(2 \pi)^{-\frac{n}{2}} \int_{\mathbb{R}^{n}} e^{i \xi x}\left(1+|\xi|^{2}\right)^{-k} d \xi \\
& =\frac{2^{1-k}}{(k-1) !}|x|^{k-\frac{n}{2}} K_{\frac{n}{2}-k}(|x|)
\end{aligned}
$$

для $k \geqslant 1$. Таким образом, легко убеждаемся, что операторы $\mathscr{G}(t)$ и $\mathscr{G}(t) \mathscr{B}:$

$$
\begin{aligned}
\mathscr{G}(t) \varphi & =\int_{\mathbb{R}^{n}} G(t, x-y) \varphi(y) d y, \\
\mathscr{G}(t) \mathscr{B} \varphi & =\int_{\mathbb{R}^{n}} H(t, x-y) \varphi(y) d y,
\end{aligned}
$$

имеют положительные ядра

$$
\begin{aligned}
& G(t, x)=(2 \pi)^{-\frac{n}{2}} e^{-\alpha t} \sum_{k=0}^{\infty} \frac{\alpha^{k} t^{k}}{k !} B_{k}(x) \geqslant 0, \\
& H(t, x)=(2 \pi)^{-\frac{n}{2}} e^{-\alpha t} \sum_{k=0}^{\infty} \frac{\alpha^{k} t^{k}}{k !} B_{k+1}(x) \geqslant 0
\end{aligned}
$$

для всех $t \geqslant 0, x \in \mathbb{R}^{n}$. Принимая во внимание оценки для функции Макдональда (см. [8]), получаем

$$
\left|B_{k}(x)\right| \leqslant\left\{\begin{array}{lc}
C|x|^{k-\frac{n+1}{2}} e^{-|x|} & \text { при }|x| \geqslant 1, \\
C \int_{|x|}^{1} y^{2 k-n-1} d y & \text { при }|x|<1
\end{array}\right.
$$

для любого $k \geqslant 1$. Отсюда следуют неравенства

$$
\begin{aligned}
\left\|\mathscr{B}^{k} \varphi\right\|_{\mathbf{L}^{p}} & \leqslant C\left\|\int_{\mathbb{R}^{n}} B_{k}(t, x-y) \varphi(y) d y\right\|_{\mathbf{L}^{p}} \leqslant\left\|B_{k}(t)\right\|_{\mathbf{L}^{1}}\|\varphi\|_{\mathbf{L}^{p}} \\
& \leqslant C\|\varphi\|_{\mathbf{L}^{p}}\left(\int_{|x|<1} d x \int_{|x|}^{1} y^{2 k-n-1} d y+\int_{|x| \geqslant 1}|x|^{k-\frac{n+1}{2}} e^{-|x|} d x\right) \\
& \leqslant C\|\varphi\|_{\mathbf{L}^{p}}
\end{aligned}
$$

для всех $1 \leqslant p \leqslant \infty$ и

$$
\begin{aligned}
& \left\|\mathscr{B}^{k} \varphi\right\|_{\mathbf{L}^{1, a}} \leqslant C\left\|\langle x\rangle^{a} \int_{\mathbb{R}^{n}} B_{k}(t, x-y) \varphi(y) d y\right\|_{\mathbf{L}^{1}} \\
& \quad \leqslant C\left\|B_{k}(t)\right\|_{\mathbf{L}^{1, a}}\|\varphi\|_{\mathbf{L}^{1, a}} \\
& \quad \leqslant C\|\varphi\|_{\mathbf{L}^{1, a}}\left(\int_{|x|<1} d x \int_{|x|}^{1} y^{2 k-n-1} d y+\int_{|x| \geqslant 1}|x|^{a+k-\frac{n+1}{2}} e^{-|x|} d x\right) \\
& \quad \leqslant C\|\varphi\|_{\mathbf{L}^{1, a}}
\end{aligned}
$$

для любых $a \geqslant 0$ и $k \geqslant 0$. 
В следующей лемме мы оценим оператор Грина $\mathscr{G}(t)$ в нормах $\|\varphi\|_{\mathbf{L}^{p}}$ и $\|\varphi\|_{\mathbf{L}^{1, a}}$, где $a \in(0,1), 1 \leqslant p \leqslant \infty$.

Лемма 1. Пусть функиия $\varphi \in \mathbf{L}^{\infty}\left(\mathbb{R}^{n}\right) \cap \mathbf{L}^{1, a}\left(\mathbb{R}^{n}\right)$, где $a \in(0,1)$. Тогда имеют место оценки

$$
\begin{gathered}
\|\mathscr{G}(t) \varphi\|_{\mathbf{L}^{p}} \leqslant e^{-a t}\|\varphi\|_{\mathbf{L}^{p}}+C t^{-\frac{n}{2}\left(\frac{1}{q}-\frac{1}{p}\right)}\|\varphi\|_{\mathbf{L}^{q}}, \\
\left\|(\Delta \mathscr{B})^{l} \mathscr{G}(t) \varphi\right\|_{\mathbf{L}^{p}} \leqslant C\langle t\rangle^{-l}\|\varphi\|_{\mathbf{L}^{p}}, \\
\left\|\mathscr{G}(t) \varphi-\vartheta G_{0}(t)\right\|_{\mathbf{L}^{\infty}} \leqslant C t^{-\frac{n}{2}-\frac{a}{2}}\left(\|\varphi\|_{\mathbf{L}^{\infty}}+\|\varphi\|_{\mathbf{L}^{1, a}}\right), \\
\left\||x|^{b}\left(\mathscr{G}(t) \varphi-\vartheta G_{0}(t)\right)\right\|_{\mathbf{L}_{x}^{1}} \leqslant C t^{\frac{b-a}{2}}\|\varphi\|_{\mathbf{L}^{1, a}}
\end{gathered}
$$

для всех $t>0$, где $G_{0}(t, x)=(4 \pi \alpha t)^{-\frac{n}{2}} e^{-\frac{|x|^{2}}{4 \alpha t}}, \quad l=0,1,2, \quad 1 \leqslant q \leqslant p \leqslant \infty$, $0 \leqslant b \leqslant a u$

$$
\vartheta=\int_{\mathbb{R}^{n}} \varphi(x) d x .
$$

ДоКАЗАТЕЛЬСтво. Заметим, что оператор Грина $\mathscr{G}(t)$ может быть представлен в виде

$$
\begin{aligned}
\mathscr{G}(t) \varphi= & \overline{\mathscr{F}}_{\xi \rightarrow x} e^{-\alpha t \frac{|\xi|^{2}}{1+|\xi|^{2}} \widehat{\varphi}(\xi)} \\
= & \overline{\mathscr{F}}_{\xi \rightarrow x} e^{-\alpha t|\xi|^{2}} \widehat{\varphi}(\xi)+e^{-\alpha t} \sum_{k=0}^{n} \frac{\alpha^{k} t^{k}}{k !} \overline{\mathscr{F}}_{\xi \rightarrow x}\left(1+|\xi|^{2}\right)^{-k} \widehat{\varphi}(\xi) \\
& +\overline{\mathscr{F}}_{\xi \rightarrow x}\left(e^{-\alpha t \frac{|\xi|^{2}}{1+|\xi|^{2}}}-e^{-\alpha t|\xi|^{2}}-e^{-\alpha t} \sum_{k=0}^{n} \frac{\alpha^{k} t^{k}}{k !}\left(1+|\xi|^{2}\right)^{-k}\right) \widehat{\varphi}(\xi) \\
= & \mathscr{G}_{0}(t) \varphi+e^{-\alpha t} \sum_{k=0}^{n} \frac{\alpha^{k} t^{k}}{k !} \mathscr{B}^{k} \varphi+\mathscr{R}(t) \varphi,
\end{aligned}
$$

где оператор Грина $\mathscr{G}_{0}(t)$ для уравнения теплопроводности

$$
\mathscr{G}_{0}(t) \varphi=\overline{\mathscr{F}} \xi \rightarrow x e^{-\alpha t|\xi|^{2}} \widehat{\varphi}(\xi)=\int_{\mathbb{R}^{n}} G_{0}(t, x-y) \varphi(y) d y
$$

имеет ядро

$$
G_{0}(t, x)=(4 \pi \alpha t)^{-\frac{n}{2}} e^{-\frac{|x|^{2}}{4 \alpha t}}
$$

а остаток

$$
\mathscr{R}(t) \varphi=\int_{\mathbb{R}^{n}} R(t, x-y) \varphi(y) d y
$$


задается ядром $R(t, x)=\overline{\mathscr{F}}_{\xi \rightarrow x} \widehat{R}(t, \xi)$, где образ Фурье $\widehat{R}(t, \xi)$ определяется следующим образом:

$$
\widehat{R}(t, \xi)=e^{-\alpha t \frac{|\xi|^{2}}{1+|\xi|^{2}}}-e^{-\alpha t|\xi|^{2}}-e^{-\alpha t} \sum_{k=0}^{n} \frac{\alpha^{k} t^{k}}{k !}\left(1+|\xi|^{2}\right)^{-k} .
$$

Ввиду неравенств (5) и (6) имеем оценки

$$
\begin{aligned}
& \left\|e^{-\alpha t} \sum_{k=0}^{n} \frac{\alpha^{k} t^{k}}{k !} \mathscr{B}^{k} \varphi\right\|_{\mathbf{L}^{p}} \leqslant C\langle t\rangle^{n} e^{-\alpha t}\|\varphi\|_{\mathbf{L}^{p}}, \\
& \left\|e^{-\alpha t} \sum_{k=0}^{n} \frac{\alpha^{k} t^{k}}{k !} \mathscr{B}^{k} \varphi\right\|_{\mathbf{L}^{1, b}} \leqslant C\langle t\rangle^{n} e^{-\alpha t}\|\varphi\|_{\mathbf{L}^{1, b}}
\end{aligned}
$$

для всех $t>0$, где $1 \leqslant p \leqslant \infty, 0 \leqslant b \leqslant a$.

Обозначим

$$
\widehat{R}_{l}(t, \xi)=\left(\frac{|\xi|^{2}}{1+|\xi|^{2}}\right)^{l} \widehat{R}(t, \xi)
$$

для $l=0,1,2$. Чтобы оценить остаток $(\Delta \mathscr{B})^{l} \mathscr{R}(t)$, запишем представление

$$
\begin{aligned}
\widehat{R}_{l}(t, \xi)= & e^{-\alpha t \frac{|\xi|^{2}}{1+|\xi|^{2}}}\left(1-e^{\left.-\alpha t \frac{|\xi|^{4}}{1+|\xi|^{2}}\right)\left(\frac{|\xi|^{2}}{1+|\xi|^{2}}\right)^{l}}\right. \\
& -e^{-\alpha t} \sum_{k=0}^{n} \frac{\alpha^{k} t^{k}}{k !}\left(1+|\xi|^{2}\right)^{-k}\left(\frac{|\xi|^{2}}{1+|\xi|^{2}}\right)^{l}
\end{aligned}
$$

при $|\xi| \leqslant 1$ и представление

$$
\begin{aligned}
\widehat{R}_{l}(t, \xi)= & -e^{-\alpha t|\xi|^{2}}\left(\frac{|\xi|^{2}}{1+|\xi|^{2}}\right)^{l} \\
& +e^{-\alpha t}\left(e^{\frac{\alpha t}{1+|\xi|^{2}}}-\sum_{k=0}^{n} \frac{\alpha^{k} t^{k}}{k !}\left(1+|\xi|^{2}\right)^{-k}\right)\left(\frac{|\xi|^{2}}{1+|\xi|^{2}}\right)^{l}
\end{aligned}
$$

при $|\xi| \geqslant 1$, откуда вытекает оценка

$$
\left|\partial_{\xi}^{j} \widehat{R}_{l}(t, \xi)\right| \leqslant C t^{\frac{j}{2}}\langle t\rangle^{-1-l} e^{-\frac{\alpha}{2} t|\xi|^{2}}+C t^{n+1} e^{-\alpha t}\left(1+|\xi|^{2}\right)^{-n-1}
$$

для всех $t>0, \xi \in \mathbb{R}^{n}, 0 \leqslant j \leqslant n+2, l=0,1,2$. Взяв обратное преобразование Фурье, для всех $|x| \leqslant 1$ найдем

$$
\begin{aligned}
\left|R_{l}(t, x)\right| & =\left|(2 \pi)^{-\frac{n}{2}} \int_{\mathbb{R}^{n}} e^{i \xi x} \widehat{R}_{l}(t, \xi) d \xi\right| \\
& \leqslant C\langle t\rangle^{-1-l}\left\|e^{-\frac{\alpha}{2} t|\xi|^{2}}\right\|_{\mathbf{L}^{1}}+C t^{n+1} e^{-\alpha t}\left\|\left(1+|\xi|^{2}\right)^{-n-1}\right\|_{\mathbf{L}^{1}} \\
& \leqslant C\langle t\rangle^{-1} t^{-\frac{n}{2}}
\end{aligned}
$$


а в случае $|x| \geqslant 1$, проведя интегрирование по частям $n+2$ раза по $\xi$, получим

$$
\begin{aligned}
\left|R_{l}(t, x)\right|= & C|x|^{-n-2}\left|\int_{\mathbb{R}^{n}} e^{i \xi x} \partial_{\xi}^{n+2} \widehat{R}_{l}(t, \xi) d \xi\right| \\
\leqslant & C|x|^{-n-2} t^{\frac{n}{2}+1}\langle t\rangle^{-1-l}\left\|e^{-\frac{\alpha}{2} t|\xi|^{2}}\right\|_{\mathbf{L}^{1}} \\
& +C|x|^{-n-2} t^{n+1} e^{-\alpha t}\left\|\left(1+|\xi|^{2}\right)^{-n-1}\right\|_{\mathbf{L}^{1}} \\
\leqslant & C\left(|x| t^{-\frac{1}{2}}\right)^{-n-2}\langle t\rangle^{-1-l} t^{-\frac{n}{2}}
\end{aligned}
$$

Таким образом, для всех $x \in \mathbb{R}^{n}, t>0$ имеем неравенство

$$
\left|R_{l}(t, x)\right| \leqslant C\left\langle x t^{-\frac{1}{2}}\right\rangle^{-n-2}\langle t\rangle^{-1-l} t^{-\frac{n}{2}} .
$$

Используя эту оценку, с помощью неравенства Юнга для сверток при $\frac{1}{p}=\frac{1}{q}+\frac{1}{r}-1$ получаем

$$
\begin{aligned}
\left\|(\Delta \mathscr{B})^{l} \mathscr{R}(t) \varphi\right\|_{\mathbf{L}^{p}} & =\left\|(2 \pi)^{-\frac{n}{2}} \int_{\mathbb{R}^{n}} R_{l}(t, x-y) \varphi(y) d y\right\|_{\mathbf{L}^{p}} \\
& \leqslant C\left\|R_{l}(t)\right\|_{\mathbf{L}^{r}}\|\varphi\|_{\mathbf{L}^{q}} \\
& \leqslant C\langle t\rangle^{-1-l} t^{-\frac{n}{2}}\left\|\left\langle x t^{-\frac{1}{2}}\right\rangle^{-n-2}\right\|_{\mathbf{L}^{r}}\|\varphi\|_{\mathbf{L}^{q}} \\
& \leqslant C\langle t\rangle^{-1-l} t^{-\frac{n}{2}+\frac{n}{2 r}}\|\varphi\|_{\mathbf{L}^{q}} \leqslant C\langle t\rangle^{-1-l} t^{-\frac{n}{2}\left(\frac{1}{q}-\frac{1}{p}\right)}\left\|_{\varphi}\right\|_{\mathbf{L}^{q}}
\end{aligned}
$$

для всех $1 \leqslant q \leqslant p \leqslant \infty$. Аналогично находим

$$
\begin{aligned}
& \left\|(\Delta \mathscr{B})^{l} \mathscr{R}(t) \varphi\right\|_{\mathbf{L}^{1, b}} \leqslant C\left\|\int_{\mathbb{R}^{n}} R_{l}(t, x-y)\langle y\rangle^{b} \varphi(y) d y\right\|_{\mathbf{L}^{1}} \\
& \quad+C\left\|\int_{\mathbb{R}^{n}}|x-y|^{b} R_{l}(t, x-y) \varphi(y) d y\right\|_{\mathbf{L}^{1}} \\
& \leqslant C\langle t\rangle^{-1-l}\|\varphi\|_{\mathbf{L}^{1, b}}+C\langle t\rangle^{-1-l} t^{-\frac{n}{2}}\left\||x|^{b}\left\langle x t^{-\frac{1}{2}}\right\rangle^{-n-2}\right\|_{\mathbf{L}^{1}}\|\varphi\|_{\mathbf{L}^{1}} \\
& \leqslant C\langle t\rangle^{-1-l}\left(\|\varphi\|_{\mathbf{L}^{1, b}}+t^{\frac{b}{2}}\|\varphi\|_{\mathbf{L}^{1}}\right)
\end{aligned}
$$

для всех $t>0,0 \leqslant b \leqslant a, l=0,1,2$. Известно, что оператор Грина $\mathscr{G}_{0}(t)$ для уравнения теплопроводности удовлетворяет оценке (см. работы [21], [24])

$$
\left\|\mathscr{G}_{0}(t) \varphi\right\|_{\mathbf{L}^{p}} \leqslant C t^{-\frac{n}{2}\left(\frac{1}{q}-\frac{1}{p}\right)}\|\varphi\|_{\mathbf{L}^{q}}
$$

для всех $1 \leqslant q \leqslant p \leqslant \infty$. Теперь из представления (7) ввиду (8) получаем

$$
\begin{aligned}
\|\mathscr{G}(t) \varphi\|_{\mathbf{L}^{p}} \leqslant & \left\|\mathscr{G}_{0}(t) \varphi\right\|_{\mathbf{L}^{p}}+\left\|e^{-\alpha t} \sum_{k=0}^{n} \frac{\alpha^{k} t^{k}}{k !} \mathscr{B}^{k} \varphi\right\|_{\mathbf{L}^{p}} \\
& +\|\mathscr{R}(t) \varphi\|_{\mathbf{L}^{p}} \leqslant C t^{-\frac{n}{2}\left(\frac{1}{q}-\frac{1}{p}\right)}\|\varphi\|_{\mathbf{L}^{q}}+C e^{-a t}\langle t\rangle^{n}\|\varphi\|_{\mathbf{L}^{p}} \\
& +C\langle t\rangle^{-1} t^{-\frac{n}{2}\left(\frac{1}{q}-\frac{1}{p}\right)}\|\varphi\|_{\mathbf{L}},
\end{aligned}
$$


откуда следует первая оценка леммы. Поскольку

$$
\left\|(\Delta \mathscr{B})^{l} \mathscr{G}_{0}(t) \varphi\right\|_{\mathbf{L}^{p}} \leqslant C\langle t\rangle^{-l}\|\varphi\|_{\mathbf{L}^{p}}
$$

то ввиду (8) справедлива вторая оценка леммы

$$
\begin{aligned}
\left\|(\Delta \mathscr{B})^{l} \mathscr{G}(t) \varphi\right\|_{\mathbf{L}^{p} \leqslant} \leqslant & \left\|(\Delta \mathscr{B})^{l} \mathscr{G}_{0}(t) \varphi\right\|_{\mathbf{L}^{p}} \\
& +\left\|(\Delta \mathscr{B})^{l} e^{-\alpha t} \sum_{k=0}^{n} \frac{\alpha^{k} t^{k}}{k !} \mathscr{B}^{k} \varphi\right\|_{\mathbf{L}^{p}} \\
& +\left\|(\Delta \mathscr{B})^{l} \mathscr{R}(t) \varphi\right\|_{\mathbf{L}^{p}} \leqslant C\langle t\rangle^{-l}\|\varphi\|_{\mathbf{L}^{p}}
\end{aligned}
$$

при $l=0,1,2$. Точно так же, используя неравенство

$$
\left\|\mathscr{G}_{0}(t) \varphi-\vartheta G_{0}(t)\right\|_{\mathbf{L}^{\infty}} \leqslant C t^{-\frac{n}{2}-\frac{a}{2}}\|\varphi\|_{\mathbf{L}^{1, a}}
$$

имеем третью оценку леммы

$$
\begin{aligned}
& \left\|\mathscr{G}(t) \varphi-\vartheta G_{0}(t)\right\|_{\mathbf{L}^{\infty}} \leqslant\left\|\mathscr{G}_{0}(t) \varphi-\vartheta G_{0}(t)\right\|_{\mathbf{L}^{\infty}} \\
& \quad+\left\|e^{-\alpha t} \sum_{k=0}^{n} \frac{\alpha^{k} t^{k}}{k !} \mathscr{B}^{k} \varphi\right\|_{\mathbf{L}^{\infty}}+\|\mathscr{R}(t) \varphi\|_{\mathbf{L}^{\infty}} \\
& \leqslant C t^{-\frac{n}{2}-\frac{a}{2}}\|\varphi\|_{\mathbf{L}^{1, a}}+C e^{-a t}\langle t\rangle^{n}\|\varphi\|_{\mathbf{L}^{\infty}+C\langle t\rangle^{-\frac{n}{2}-1}\|\varphi\|_{\mathbf{L}^{1}}} \leqslant C t^{-\frac{n}{2}-\frac{a}{2}}\left(\|\varphi\|_{\mathbf{L}^{\infty}}+\|\varphi\|_{\mathbf{L}^{1, a}}\right),
\end{aligned}
$$

поскольку $\|\varphi\|_{\mathbf{L}^{1}} \leqslant\left\|\langle\cdot\rangle^{a} \varphi\right\|_{\mathbf{L}^{1}}=\|\varphi\|_{\mathbf{L}^{1, a}}$. Наконец, учитьвая неравенство

$$
\left\||x|^{b}\left(\mathscr{G}_{0}(t) \varphi-\vartheta G_{0}(t)\right)\right\|_{\mathbf{L}_{x}^{1}} \leqslant C t^{\frac{b-a}{2}}\|\varphi\|_{\mathbf{L}^{1, a}}
$$

в силу (9) находим

$$
\begin{aligned}
\left\||x|^{b}\left(\mathscr{G}(t) \varphi-\vartheta G_{0}(t)\right)\right\|_{\mathbf{L}_{x}^{1}} \leqslant\left\||x|^{b}\left(\mathscr{G}_{0}(t) \varphi-\vartheta G_{0}(t)\right)\right\|_{\mathbf{L}_{x}^{1}} \\
\quad+\left\|e^{-\alpha t} \sum_{k=0}^{n} \frac{\alpha^{k} t^{k}}{k !} \mathscr{B}^{k} \varphi\right\|_{\mathbf{L}^{1, b}}+\|\mathscr{R}(t) \varphi\|_{\mathbf{L}^{1, b}} \\
\leqslant C t^{\frac{b-a}{2}}\|\varphi\|_{\mathbf{L}^{1, a}}+C e^{-a t}\langle t\rangle^{n}\|\varphi\|_{\mathbf{L}^{1, a}} \\
\quad+C\langle t\rangle^{-1}\left(\|\varphi\|_{\mathbf{L}^{1, a}}+\langle t\rangle^{\frac{b}{2}}\|\varphi\|_{\mathbf{L}^{1}}\right) \leqslant C t^{\frac{b-a}{2}}\|\varphi\|_{\mathbf{L}^{1, a}},
\end{aligned}
$$

откуда следует последняя оценка леммы. Лемма доказана.

В следуюшей лемме мы оценим оператор Грина в основной норме

$$
\|\varphi\|_{\mathbf{X}}=\sup _{t>0}\left(\langle t\rangle^{\frac{n}{2}}\|\varphi(t)\|_{\mathbf{L}^{\infty}}+\langle t\rangle^{-\frac{a}{2}}\|\varphi(t)\|_{\mathbf{L}^{1, a}}\right)
$$


где $a \in(0,1)$. Отметим, что норма $\mathbf{L}^{1}$ оценивается через норму $\mathbf{X}$ :

$$
\begin{aligned}
\|\varphi(t)\|_{\mathbf{L}^{1}} & =\int_{|x| \leqslant\langle t)^{\frac{1}{2}}}|\varphi(t, x)| d x+\int_{|x|>\langle t\rangle^{\frac{1}{2}}}|x|^{-a}|x|^{a}|\varphi(t, x)| d x \\
& \leqslant C\langle t\rangle^{\frac{n}{2}}\|\varphi(t)\|_{\mathbf{L}^{\infty}}+C\langle t\rangle^{-\frac{a}{2}}\|\varphi(t)\|_{\mathbf{L}^{1, a}} \leqslant C\|\varphi\| \mathbf{X} .
\end{aligned}
$$

Определим функцию

$$
g(t)=1+\kappa \log \langle t\rangle
$$

с некоторым $\kappa>0$.

ЛЕмма 2. Пусть функиия $f(t, x)$ имеет нулевое среднее значение

$$
\int_{\mathbb{R}^{n}} f(t, x) d x=0
$$

Тогда справедливо следующее неравенство:

$$
\left\|g^{k}(t) \int_{0}^{t} g^{-k}(\tau) \mathscr{G}(t-\tau) \mathscr{B} f(\tau) d \tau\right\|_{\mathbf{X}} \leqslant C\|\langle t\rangle f\|_{\mathbf{X}}
$$

при $k=0,1$, в предположении, что правая часть ограничена.

ДоКАЗАТЕЛЬСТВО. В силу оценки $g^{-1}(\tau) \leqslant C$ с помощью леммы 1 получим

$$
\begin{aligned}
& \left\|\int_{0}^{t} g^{-k}(\tau) \mathscr{G}(t-\tau) f(\tau) d \tau\right\|_{\mathbf{L}^{\infty}}+\left\|\int_{0}^{t} g^{-k}(\tau) \mathscr{G}(t-\tau) f(\tau) d \tau\right\|_{\mathbf{L}^{1, a}} \\
& \quad \leqslant C\|\langle t\rangle f\|_{\mathbf{X}} \leqslant C\|\langle t\rangle f\|_{\mathbf{X}} g^{-k}(t)
\end{aligned}
$$

для всех $0 \leqslant t \leqslant 4$. Теперь рассмотрим $t>4$. Из определения функции $g(t)$ имеем оценку $\langle t\rangle^{-\frac{a}{4}} \leqslant C g^{-1}(t)$, а также

$$
\begin{aligned}
\sup _{\tau \in[\sqrt{t}, t]} g^{-1}(\tau) & \leqslant C(1+\kappa \log (1+\sqrt{t}))^{-1} \\
& \leqslant C\left(1+\frac{\kappa}{2} \log \langle t\rangle\right)^{-1} \leqslant C g^{-1}(t),
\end{aligned}
$$

поэтому с помощью леммы 1 ввиду оценок (5) и (6) получаем

$$
\begin{aligned}
& \left\|\int_{0}^{t} g^{-k}(\tau) \mathscr{G}(t-\tau) \mathscr{B} f(\tau) d \tau\right\|_{\mathbf{L}^{\infty}} \\
& \leqslant C \int_{0}^{\sqrt{t}}(t-\tau)^{-\frac{n}{2}-\frac{a}{2}}\left(\|\mathscr{B} f(\tau)\|_{\mathbf{L}^{\infty}}+\|\mathscr{B} f(\tau)\|_{\mathbf{L}^{1, a}}\right) d \tau \\
& \quad+C g^{-k}(t) \int_{\sqrt{t}}^{\frac{t}{2}}(t-\tau)^{-\frac{n}{2}-\frac{a}{2}}\left(\|\mathscr{B} f(\tau)\|_{\mathbf{L}^{\infty}}+\|\mathscr{B} f(\tau)\|_{\mathbf{L}^{1, a}}\right) d \tau \\
& \quad+C g^{-k}(t) \int_{\frac{t}{2}}^{t}\|\mathscr{B} f(\tau)\|_{\mathbf{L}^{\infty}} d \tau
\end{aligned}
$$


откуда, используя определение нормы $\mathbf{X}$, находим

$$
\begin{aligned}
& \left\|\int_{0}^{t} g^{-k}(\tau) \mathscr{G}(t-\tau) \mathscr{B} f(\tau) d \tau\right\|_{\mathbf{L}^{\infty}} \\
& \leqslant C\|\langle t\rangle f\| \mathbf{x} \int_{0}^{\sqrt{t}}(t-\tau)^{-\frac{n}{2}-\frac{a}{2}}\langle\tau\rangle^{\frac{a}{2}-1} d \tau \\
& +C g^{-k}(t)\|\langle t\rangle f\|_{\mathbf{X}} \int_{\sqrt{t}}^{\frac{t}{2}}(t-\tau)^{-\frac{n}{2}-\frac{a}{2}}\langle\tau\rangle^{\frac{a}{2}-1} d \tau \\
& +C g^{-k}(t)\|\langle t\rangle f\|_{\mathbf{X}} \int_{\frac{t}{2}}^{t}\langle\tau\rangle^{-\frac{n}{2}-1} d \tau \\
& \leqslant C\left(t^{-\frac{n}{2}-\frac{a}{4}}+g^{-k}(t) t^{-\frac{n}{2}}\right)\|\langle t\rangle f\|_{\mathbf{X}} \leqslant C g^{-k}(t) t^{-\frac{n}{2}}\|\langle t\rangle f\| \mathbf{X} .
\end{aligned}
$$

Аналогично оценим

$$
\begin{aligned}
& \left\|\int_{0}^{t} g^{-k}(\tau) \mathscr{G}(t-\tau) \mathscr{B} f(\tau) d \tau\right\|_{\mathbf{L}^{1, a}} \\
& \quad \leqslant C \int_{0}^{t} g^{-k}(\tau)\|\mathscr{B} f(\tau)\|_{\mathbf{L}^{1, a}} d \tau \\
& \quad \leqslant C\|\langle t\rangle f\|_{\mathbf{X}} \int_{0}^{\sqrt{t}} \tau^{\frac{a}{2}-1} d \tau+C g^{-k}(t)\|\langle t\rangle f\|_{\mathbf{X}} \int_{\sqrt{t}}^{t} \tau^{\frac{a}{2}-1} d \tau \\
& \quad \leqslant C \varepsilon\left(t^{\frac{a}{4}}+g^{-k}(t) t^{\frac{a}{2}}\right)\|\langle t\rangle f\|_{\mathbf{X}} \leqslant C g^{-k}(t) t^{\frac{a}{2}}\|\langle t\rangle f\|_{\mathbf{X}}
\end{aligned}
$$

для всех $t>4$, откуда следует результат леммы, поскольку

$$
\begin{aligned}
\| g^{k}(t) & \int_{0}^{t} g^{-k}(\tau) \mathscr{G}(t-\tau) \mathscr{B} f(\tau) d \tau \|_{\mathbf{X}} \\
= & \sup _{t>0}\left(\langle t\rangle^{\frac{n}{2}} g^{k}(t)\left\|\int_{0}^{t} g^{-k}(\tau) \mathscr{G}(t-\tau) \mathscr{B} f(\tau) d \tau\right\|_{\mathbf{L}^{\infty}}\right. \\
& \left.+\langle t\rangle^{-\frac{a}{2}} g^{k}(t)\left\|\int_{0}^{t} g^{-k}(\tau) \mathscr{G}(t-\tau) \mathscr{B} f(\tau) d \tau\right\|_{\mathbf{L}^{1, a}}\right) \leqslant C\|\langle t\rangle f\|_{\mathbf{X}}
\end{aligned}
$$

Лемма доказана.

2.2. Локальное существование. Докажем локальное существование решений задачи Коши (1). Под решением задачи Коши (1) далее будем понимать решение соответствующего интегрального уравнения

$$
u(t)=\mathscr{G}(t) u_{0}+\lambda \int_{0}^{t} \mathscr{G}(t-\tau) \mathscr{B}|u(\tau)|^{\sigma} u(\tau) d \tau .
$$

УТВЕРЖДЕНИЕ 1. Пусть начальные данные $u_{0} \in \mathbf{C}\left(\mathbb{R}^{n}\right) \cap \mathbf{L}^{\infty}\left(\mathbb{R}^{n}\right) \cap \mathbf{L}^{1, a}\left(\mathbb{R}^{n}\right)$, $a \geqslant 0$. Тогда для некоторого $T>0$ существует единственное решение $u \in \mathbf{C}\left([0, T] ; \mathbf{C}\left(\mathbb{R}^{n}\right) \cap \mathbf{L}^{\infty}\left(\mathbb{R}^{n}\right) \cap \mathbf{L}^{1, a}\left(\mathbb{R}^{n}\right)\right)$ задачи Коши (1). 
ДокАЗАТЕЛЬСтво. Применим принцип сжимающих отображений в функциональном пространстве

$$
\mathbf{Z}=\left\{\varphi \in \mathbf{C}\left([0, T] ; \mathbf{C}\left(\mathbb{R}^{n}\right) \cap \mathbf{L}^{\infty}\left(\mathbb{R}^{n}\right) \cap \mathbf{L}^{1, a}\left(\mathbb{R}^{n}\right)\right):\|u\|_{\mathbf{Z}}<\infty\right\}
$$

с нормой

$$
\|u\|_{\mathbf{Z}}=\sup _{t \in[0, T]}\left(\|u(t)\|_{\mathbf{L}^{1, a}}+\|u(t)\|_{\mathbf{L}^{\infty}}\right) .
$$

Для $v \in \mathbf{Z}$ определим отображение $\mathscr{M}(v)$ следуюшим образом:

$$
\mathscr{M}(v)=\mathscr{G}(t) u_{0}+\lambda \int_{0}^{t} \mathscr{G}(t-\tau) \mathscr{B}|v(\tau)|^{\sigma} v(\tau) d \tau .
$$

В предположении, что $\|v\|_{\mathbf{z}}<\delta$, докажем, что $\|\mathscr{M}(v)\|_{\mathbf{z}}<\delta$.

Применяя первую оценку леммы 1 , находим

$$
\begin{aligned}
\|\mathscr{M}(v)\|_{\mathbf{L}^{\infty}} & \leqslant\left\|\mathscr{G}(t) u_{0}\right\|_{\mathbf{L}^{\infty}}+|\lambda| \int_{0}^{t}\left\|\mathscr{G}(t-\tau) \mathscr{B}|v(\tau)|^{\sigma} v(\tau)\right\|_{\mathbf{L}^{\infty}} d \tau \\
& \leqslant C\left\|u_{0}\right\|_{\mathbf{L}^{\infty}}+C \int_{0}^{t}\left\|\mathscr{B}|v(\tau)|^{\sigma} v(\tau)\right\|_{\mathbf{L} \infty} d \tau .
\end{aligned}
$$

Поскольку в силу четвертой оценки леммы 1 имеем

$$
\|\mathscr{G}(t) \varphi\|_{\mathbf{L}^{1, a}} \leqslant C\langle t\rangle^{\frac{a}{2}}\|\varphi\|_{\mathbf{L}^{1, a}},
$$

Tо

$$
\begin{aligned}
\|\mathscr{M}(v)\|_{\mathbf{L}^{1, a}} & \leqslant\left\|\mathscr{G}(t) u_{0}\right\|_{\mathbf{L}^{1, a}}+|\lambda| \int_{0}^{t}\left\|\mathscr{G}(t-\tau) \mathscr{B}|v(\tau)|^{\sigma} v(\tau)\right\|_{\mathbf{L}^{1, a}} d \tau \\
& \leqslant C\langle t\rangle^{\frac{a}{2}}\left\|u_{0}\right\|_{\mathbf{L}^{1, a}}+C \int_{0}^{t}\langle t-\tau\rangle^{\frac{a}{2}}\left\|\mathscr{B}|v(\tau)|^{\sigma} v(\tau)\right\|_{\mathbf{L}^{1, a}} d \tau
\end{aligned}
$$

В силу (5), (6) оценим

$$
\begin{gathered}
\left\|\mathscr{B}|v(\tau)|^{\sigma} v(\tau)\right\|_{\mathbf{L}^{\infty}} \leqslant C\left\||v(\tau)|^{\sigma+1}\right\|_{\mathbf{L}^{\infty}} \\
\quad \leqslant C\|v(\tau)\|_{\mathbf{L}^{\infty}}^{\sigma+1} \leqslant C\|v(\tau)\|_{\mathbf{Z}}^{\sigma+1} \leqslant C \delta^{\sigma+1} \\
\left\|\mathscr{B}|v(\tau)|^{\sigma} v(\tau)\right\|_{\mathbf{L}^{1, a}} \leqslant C\|v(\tau)\|_{\mathbf{L}^{1, a}}^{\sigma+1} \\
\leqslant C\|v(\tau)\|_{\mathbf{L}^{\infty}}^{\sigma}\|v(\tau)\|_{\mathbf{L}^{1, a}} \leqslant C\|v(\tau)\|_{\mathbf{Z}}^{\sigma+1} \leqslant C \delta^{\sigma+1} .
\end{gathered}
$$

Поэтому из (12) и (13) получим

$$
\begin{gathered}
\|\mathscr{M}(v)\|_{\mathbf{L}^{\infty}} \leqslant C\left\|u_{0}\right\|_{\mathbf{L}^{\infty}}+C T \delta^{\sigma+1}, \\
\|\mathscr{M}(v)\|_{\mathbf{L}^{1, a}} \leqslant C\langle T\rangle^{\frac{a}{2}}\left\|u_{0}\right\|_{\mathbf{L}^{1, a}}+C T\langle T\rangle^{\frac{a}{2}} \delta^{\sigma+1} .
\end{gathered}
$$


Таким образом, если выбрать $\delta \geqslant 2 C\left\|u_{0}\right\| \mathbf{z}$ и достаточно малое $T>0$, то

$$
\|\mathscr{M}(v)\|_{\mathbf{z}} \leqslant \delta .
$$

Значит, отображение $\mathscr{M}$ преобразует шар радиуса $\delta>0$ в пространстве $\mathbf{Z}$ в себя. Теперь докажем, что справедлива оценка для разности

$$
\left\|\mathscr{M}\left(v_{1}\right)-\mathscr{M}\left(v_{2}\right)\right\|_{\mathbf{z}} \leqslant \frac{1}{2}\left\|v_{1}-v_{2}\right\|_{\mathbf{z}}
$$

Имеем

$$
\left\|\mathscr{M}\left(v_{1}\right)-\mathscr{M}\left(v_{2}\right)\right\|_{\mathbf{z}} \leqslant C \int_{0}^{T}\left\|\mathscr{B}\left(\left|v_{1}\right|^{\sigma} v_{1}(\tau)-\left|v_{2}\right|^{\sigma} v_{2}(\tau)\right)\right\|_{\mathbf{Z}}\langle t-\tau\rangle^{\frac{a}{2}} d \tau .
$$

Поскольку

$$
\left\|\mathscr{B}\left(\left|v_{1}\right|^{\sigma} v_{1}(\tau)-\left|v_{2}\right|^{\sigma} v_{2}(\tau)\right)\right\|_{\mathbf{Z}} \leqslant C \delta^{\sigma}\left\|v_{1}-v_{2}\right\|_{\mathbf{z}}
$$

то получаем

$$
\left\|\mathscr{M}\left(v_{1}\right)-\mathscr{M}\left(v_{2}\right)\right\|_{\mathbf{z}} \leqslant C T \delta^{\sigma}\left\|v_{1}-v_{2}\right\|_{\mathbf{z}} \leqslant \frac{1}{2}\left\|v_{1}-v_{2}\right\|_{\mathbf{z}},
$$

если взять достаточно малое время существования $T>0$.

Итак, $\mathscr{M}$ является сжимающим отображением, поэтому существует единственное решение $u(t, x) \in \mathbf{C}\left([0, T] ; \mathbf{C}\left(\mathbb{R}^{n}\right) \cap \mathbf{L}^{\infty}\left(\mathbb{R}^{n}\right) \cap \mathbf{L}^{1, a}\left(\mathbb{R}^{n}\right)\right)$ задачи (1). У тверждение доказано.

\section{§3. Асимптотически слабая нелинейность}

3.1. Малые начальные данные. Чтобы доказать глобальное существование решения в случае произвольного знака коэффициента $\lambda$ перед нелинейностью, нам приходится предположить малость начальных данных. (В случае $\lambda>0$ возможно разрушение решений за конечное время (см. книгу [36].) Также нам необходимо следить за скоростью убывания нелинейного члена. В суперкритическом случае $\sigma>\frac{2}{n}$ нелинейность $|u|^{\sigma} u$ оказывается асимптотически слабой в том смысле, что она не имеет существенного влияния на вид главного члена асимптотики решения при больших временах.

Tеорема 1. Пусть $\sigma>\frac{2}{n}$. Предположим, что начальные данные $u_{0} \in$ $\mathbf{C}\left(\mathbb{R}^{n}\right) \cap \mathbf{L}^{\infty}\left(\mathbb{R}^{n}\right) \cap \mathbf{L}^{1, a}\left(\mathbb{R}^{n}\right), \quad a \in(0,1]$, имеют достаточно малую норму $\left\|u_{0}\right\|_{\mathbf{L}^{1, a}}+\left\|u_{0}\right\|_{\mathbf{L}} \infty$. Тогда существует единственное решение $u \in \mathbf{C}([0, \infty)$; $\left.\mathbf{C}\left(\mathbb{R}^{n}\right) \cap \mathbf{L}^{\infty}\left(\mathbb{R}^{n}\right) \cap \mathbf{L}^{1, a}\left(\mathbb{R}^{n}\right)\right)$ задачи Коши (1). Это решение имеет асимптотику

$$
u(t, x)=A(\alpha t)^{-\frac{n}{2}} e^{-\frac{|x|^{2}}{4 \alpha t}}+O\left(t^{-\frac{n}{2}-\gamma}\right)
$$

при $t \rightarrow \infty$ равномерно по $x \in \mathbb{R}^{n}$, әде $0<\gamma<\min \left(\frac{a}{2}, \frac{n}{2} \sigma-1\right)$, константа $A$ задается следуюшим образом:

$$
\begin{aligned}
A & =\int_{\mathbb{R}^{n}} u_{0}(x) d x+\lambda \int_{0}^{\infty} d t \int_{\mathbb{R}^{n}} \mathscr{B}|u(t, x)|^{\sigma} u(t, x) d x \\
& =\lim _{t \rightarrow+\infty} \int_{\mathbb{R}^{n}} u(t, x) d x .
\end{aligned}
$$


ЗАмечАниЕ 1. Частный случай теоремы 1 был рассмотрен в заметке [46].

ДоКАЗАТЕЛЬСТво. Применим принцип сжимающих отображений в функциональном пространстве

$$
\mathbf{X}=\left\{\varphi \in \mathbf{C}\left([0, \infty) ; \mathbf{C}\left(\mathbb{R}^{n}\right) \cap \mathbf{L}^{\infty}\left(\mathbb{R}^{n}\right) \cap \mathbf{L}^{1, a}\left(\mathbb{R}^{n}\right)\right):\|\varphi\| \mathbf{X}<\infty\right\},
$$

где

$$
\|\varphi\|_{\mathbf{X}}=\sup _{t>0}\left(\langle t\rangle^{-\frac{a}{2}}\|\varphi(t)\|_{\mathbf{L}^{1, a}}+\langle t\rangle^{\frac{n}{2}}\|\varphi(t)\|_{\mathbf{L}^{\infty}}\right) .
$$

Отметим, что $\sup _{t>0}\|\varphi(t)\|_{\mathbf{L}^{1}} \leqslant\|\varphi\|_{\mathbf{X}}$.

Пусть $\|v\|_{\mathbf{x}}<\delta$, где $\delta>0$ достаточно мало. Определим отображение $\mathscr{M}(v)$ с помощью формулы (11). Сначала докажем, что

$$
\|\mathscr{M}(v)\|_{\mathbf{x}}<\delta .
$$

Применяя первую оценку леммы 1 при $p=\infty, q=1$, получаем

$$
\|\mathscr{G}(t) \varphi\|_{\mathbf{L}^{\infty}} \leqslant C t^{-\frac{n}{2}}\left(\|\varphi\|_{\mathbf{L}^{\infty}}+\|\varphi\|_{\mathbf{L}^{1}}\right),
$$

а из второй оценки леммы 1 при $p=\infty, l=0$ следует, что

$$
\|\mathscr{G}(t) \varphi\|_{\mathbf{L} \infty} \leqslant C\|\varphi\|_{\mathbf{L}} \infty,
$$

поэтому

$$
\begin{aligned}
& \|\mathscr{M}(v)\|_{\mathbf{L}^{\infty}} \leqslant C\langle t\rangle^{-\frac{n}{2}}\left(\left\|u_{0}\right\|_{\mathbf{L}^{\infty}}+\left\|u_{0}\right\|_{\mathbf{L}^{1}}\right) \\
& +C \int_{0}^{\frac{t}{2}}\langle t-\tau\rangle^{-\frac{n}{2}}\left(\left\|\mathscr{B}|v(\tau)|^{\sigma} v(\tau)\right\|_{\mathbf{L} \infty}+\left\|\mathscr{B}|v(\tau)|^{\sigma} v(\tau)\right\|_{\mathbf{L}^{1}}\right) d \tau \\
& +C \int_{\frac{t}{2}}^{t}\left\|\mathscr{B}|v(\tau)|^{\sigma} v(\tau)\right\|_{\mathbf{L} \infty} d \tau
\end{aligned}
$$

Используя неравенства

$$
\begin{aligned}
\left\|\mathscr{B}|v(\tau)|^{\sigma} v(\tau)\right\|_{\mathbf{L}^{\infty}} & \leqslant C\left\||v(\tau)|^{\sigma+1}\right\|_{\mathbf{L}^{\infty}} \\
& \leqslant C\|v(\tau)\|_{\mathbf{L}^{\infty}}^{\sigma+1} \leqslant C \delta^{\sigma+1}\langle\tau\rangle^{-\frac{n}{2}(\sigma+1)}, \\
\left\|\mathscr{B}|v(\tau)|^{\sigma} v(\tau)\right\|_{\mathbf{L}^{1}} & \leqslant C\left\|\left.v(\tau)\right|^{\sigma+1}\right\|_{\mathbf{L}^{1}} \\
& \leqslant C\|v(\tau)\|_{\mathbf{L}^{\infty}}^{\sigma}\|v(\tau)\|_{\mathbf{L}^{1}} \leqslant C \delta^{\sigma+1}\langle\tau\rangle^{-\frac{n}{2} \sigma},
\end{aligned}
$$

находим

$$
\begin{aligned}
\|\mathscr{M}(v)\|_{\mathbf{L}^{\infty} \leqslant} \leqslant & C\langle t\rangle^{-\frac{n}{2}}\left(\left\|u_{0}\right\|_{\mathbf{L}^{\infty}}+\left\|u_{0}\right\|_{\mathbf{L}^{1}}\right) \\
& +C \delta^{\sigma+1} \int_{0}^{\frac{t}{2}}\langle\tau\rangle^{-\frac{n}{2} \sigma}\langle t-\tau\rangle^{-\frac{n}{2}} d \tau \\
& +C \delta^{\sigma+1} \int_{\frac{t}{2}}^{t}\langle\tau\rangle^{-\frac{n}{2}(\sigma+1)} d \tau \leqslant \delta\langle t\rangle^{-\frac{n}{2}}
\end{aligned}
$$


поскольку начальные данные $u_{0}$ и $\delta>0$ являются малыми, и в суперкритическом случае имеем $\frac{n}{2} \sigma>1$. Подобным образом оценим норму

$$
\begin{aligned}
\|\mathscr{M}(v)\|_{\mathbf{L}^{1, a}} \leqslant & C\langle t\rangle^{\frac{a}{2}}\left(\left\|u_{0}\right\|_{\mathbf{L}^{\infty}}+\left\|u_{0}\right\|_{\mathbf{L}^{1}}\right) \\
& +C \int_{0}^{t}\left\|\mathscr{B}|v(\tau)|^{\sigma} v(\tau)\right\|_{\mathbf{L}^{1, a}} d \tau+C \int_{0}^{t}\left\|\mathscr{B}|v(\tau)|^{\sigma} v(\tau)\right\|_{\mathbf{L}^{1}}\langle t-\tau\rangle^{\frac{a}{2}} d \tau \\
\leqslant & \delta\langle t\rangle^{\frac{a}{2}}+C \delta^{\sigma+1} \int_{0}^{t}\langle\tau\rangle^{-\frac{n}{2} \sigma+\frac{a}{2}} d \tau \\
& +C \delta^{\sigma+1} \int_{0}^{t}\langle\tau\rangle^{-\frac{n}{2} \sigma}\langle t-\tau\rangle^{\frac{a}{2}} d \tau \leqslant \delta\langle t\rangle^{\frac{a}{2}}
\end{aligned}
$$

где мы учли неравенство

$$
\begin{aligned}
\left\|\mathscr{B}|v(\tau)|^{\sigma} v(\tau)\right\|_{\mathbf{L}^{1, a}} & \leqslant C\left\||v(\tau)|^{\sigma+1}\right\|_{\mathbf{L}^{1, a}} \\
& \leqslant C\|v(\tau)\|_{\mathbf{L}^{\infty}}^{\sigma}\|v(\tau)\|_{\mathbf{L}^{1, a}} \leqslant C \delta^{\sigma+1}\langle\tau\rangle^{-\frac{n}{2} \sigma+\frac{a}{2}}
\end{aligned}
$$

Таким образом, $\|\mathscr{M}(v)\| \mathbf{x} \leqslant \delta$, откуда следует, что отображение $\mathscr{M}$ преобразует шар радиуса $\delta>0$ в пространстве $\mathbf{X}$ в себя. Аналогично оценивается разность

$$
\left\|\mathscr{M}\left(v_{1}\right)-\mathscr{M}\left(v_{2}\right)\right\|_{\mathbf{x}} \leqslant \frac{1}{2}\left\|v_{1}-v_{2}\right\|_{\mathbf{x}}
$$

откуда следует, что $\mathscr{M}$ является сжимающим отображением. Таким образом, существует единственное решение $u(t, x) \in \mathbf{C}\left([0, \infty) ; \mathbf{C}\left(\mathbb{R}^{n}\right) \cap \mathbf{L}^{\infty}\left(\mathbb{R}^{n}\right) \cap \mathbf{L}^{1, a}\left(\mathbb{R}^{n}\right)\right)$ задачи (1), подчиняющееся оценкам

$$
\|u(t)\|_{\mathbf{L}^{\infty}} \leqslant C\langle t\rangle^{-\frac{n}{2}}, \quad\|u(t)\|_{\mathbf{L}^{1, a}} \leqslant C\langle t\rangle^{\frac{a}{2}} .
$$

Теперь докажем асимптотику (14). Воспользуемся интегральным уравнением (10). Ввиду третьей оценки леммы 1 имеем следующее асимптотическое представление для оператора Грина:

$$
\mathscr{G}(t) u_{0}=\theta G_{0}(t, x)+O\left(t^{-\frac{n+a}{2}}\left\|u_{0}\right\|_{\mathbf{L}^{1, a}}\right)
$$

при $t \rightarrow \infty$ равномерно по $x \in \mathbb{R}^{n}$, где $G_{0}(t, x)=(4 \pi \alpha t)^{-\frac{n}{2}} e^{-\frac{|x|^{2}}{4 \alpha t}}-$ функция Грина для уравнения теплопроводности,

$$
\theta=\int_{\mathbb{R}^{n}} u_{0}(x) d x
$$

- обшая масса начальных данных, $a \in(0,1]$. Рассмотрим теперь разность

$$
\begin{aligned}
\lambda \int_{0}^{t} \mathscr{G}(t-\tau) \mathscr{B}|u(\tau)|^{\sigma} u(\tau) d \tau-G_{0}(t, x) \int_{0}^{\infty} \vartheta(\tau) d \tau \\
=\lambda \int_{0}^{t}\left(\mathscr{G}(t-\tau) \mathscr{B}|u(\tau)|^{\sigma} u(\tau)-\vartheta(\tau) G_{0}(t-\tau, x)\right) d \tau \\
\quad+\int_{0}^{t}\left(G_{0}(t-\tau, x)-G_{0}(t, x)\right) \vartheta(\tau) d \tau+G_{0}(t, x) \int_{t}^{\infty} \vartheta(\tau) d \tau
\end{aligned}
$$


где

$$
\vartheta(\tau)=\lambda \int_{\mathbb{R}^{n}} \mathscr{B}|u(\tau)|^{\sigma} u(\tau) d y .
$$

Пользуясь леммой 1 , получаем

$$
\begin{aligned}
& \left\|\lambda \mathscr{G}(t-\tau) \mathscr{B}|u(\tau)|^{\sigma} u(\tau)-\vartheta(\tau) G_{0}(t-\tau, x)\right\|_{\mathbf{L} \infty} \\
& \quad \leqslant C(t-\tau)^{-\frac{n}{2}-\frac{a}{2}}\left(\left\|\mathscr{B}|u|^{\sigma} u(\tau)\right\|_{\left.\mathbf{L}^{\infty}+\left\|\mathscr{B}|u|^{\sigma} u(\tau)\right\|_{\mathbf{L}^{1, a}}\right)}\right.
\end{aligned}
$$

в области $0<\tau<\frac{t}{2}$, а в области $\frac{t}{2} \leqslant \tau<t$ имеем оценку

$$
\begin{aligned}
& \left\|\lambda \mathscr{G}(t-\tau) \mathscr{B}|u(\tau)|^{\sigma} u(\tau)-\vartheta(\tau) G_{0}(t-\tau, x)\right\|_{\mathbf{L}^{\infty}} \\
& \quad \leqslant C\left\|\mathscr{B}|u|^{\sigma} u(\tau)\right\|_{\mathbf{L}^{\infty}} .
\end{aligned}
$$

Таким образом, ввиду (15) имеем

$$
\begin{aligned}
& \left\|\int_{0}^{t}\left(\lambda \mathscr{G}(t-\tau) \mathscr{B}|u(\tau)|^{\sigma} u(\tau)-\vartheta(\tau) G_{0}(t-\tau, x)\right) d \tau\right\|_{\mathbf{L}^{\infty}} \\
& \quad \leqslant C\|u\|_{\mathbf{X}}^{\sigma+1} \int_{0}^{\frac{t}{2}}(t-\tau)^{-\frac{n}{2}-\frac{a}{2}}\langle\tau\rangle^{-\frac{n}{2} \sigma} d \tau+C\|u\|_{\mathbf{X}}^{\sigma+1} \int_{\frac{t}{2}}^{t}\langle\tau\rangle^{-\frac{n}{2}(\sigma+1)} d \tau \\
& \quad \leqslant C\langle t\rangle^{-\frac{n}{2}-\gamma}
\end{aligned}
$$

где $0<\gamma<\min \left(\frac{a}{2}, \frac{n}{2} \sigma-1\right)$. Теперь оценим разность

$$
\begin{aligned}
& \left\|\int_{0}^{t}\left(G_{0}(t-\tau, x)-G_{0}(t, x)\right) \vartheta(\tau) d \tau\right\|_{\mathbf{L}^{\infty}} \\
& \quad \leqslant C \int_{0}^{t}\left\|G_{0}(t-\tau)-G_{0}(t)\right\|_{\mathbf{L}^{\infty}\langle\tau\rangle^{-\frac{n}{2} \sigma} d \tau} \\
& \quad \leqslant C \int_{0}^{\frac{t}{2}}\langle t-\tau\rangle^{-\frac{n}{2}-\gamma}\langle\tau\rangle^{\gamma-\frac{n}{2} \sigma} d \tau+C \int_{\frac{t}{2}}^{t}\langle t-\tau\rangle^{-\frac{n}{2}}\langle\tau\rangle^{-\frac{n}{2} \sigma} d \tau \\
& \quad \leqslant C\langle t\rangle^{-\frac{n}{2}-\gamma},
\end{aligned}
$$

а для последнего слагаемого в (17) имеем оценку

$$
\left\|G_{0}(t) \int_{t}^{\infty} \vartheta(\tau) d \tau\right\|_{\mathbf{L}^{\infty}} \leqslant C t^{-\frac{n}{2}} \int_{t}^{\infty}\langle\tau\rangle^{-\frac{n}{2} \sigma} d \tau \leqslant C\langle t\rangle^{-\frac{n}{2}-\gamma} .
$$

Таким образом, из интегрального уравнения (10) мы видим, что существует число

$$
A=\theta+\int_{0}^{\infty} \vartheta(\tau) d \tau=\lim _{t \rightarrow+\infty} \int_{\mathbb{R}^{n}} u(t, x) d x
$$

такое, что имеет место асимптотика (14). Теорема доказана. 
3.2. Большие начальные данные. Рассмотрим случай $\lambda<0$. Тогда, следуя методу работы [23], мы сможем отказаться от малости начальных данных $u_{0}(x)$.

Теорема 2. Пусть $\sigma>\frac{2}{n}, \lambda<0, n=1,2$. Предположим, что начальные данные $u_{0} \in \mathbf{W}_{\infty}^{2}\left(\mathbb{R}^{n}\right) \cap \mathbf{W}_{1}^{2, a}\left(\mathbb{R}^{n}\right), a \in(0,1]$. Тогда существует единственное решение $u \in \mathbf{C}\left([0, \infty) ; \mathbf{C}\left(\mathbb{R}^{n}\right) \cap \mathbf{L}^{\infty}\left(\mathbb{R}^{n}\right) \cap \mathbf{L}^{1, a}\left(\mathbb{R}^{n}\right)\right)$ задачи Кочи (1), причем справедлива асимптотика (14).

Прежде чем доказывать теорему 2, мы докажем несколько лемм. Определим норму

$$
\|\varphi\|_{p, q} \equiv\|\| \varphi(t, x)\left\|_{\mathbf{L}^{q}\left(\mathbb{R}_{x}^{n}\right)}\right\|_{\mathbf{L}^{p}\left(\mathbb{R}_{t}^{+}\right)} .
$$

ЛЕмма 3. Пусть размерность $n=1,2$. Предположим, что начальнье даннье $u_{0} \in \mathbf{W}_{\infty}^{2}\left(\mathbb{R}^{n}\right) \cap \mathbf{W}_{1}^{2}\left(\mathbb{R}^{n}\right)$. Пусть нормы $\|u\|_{\infty, 2} u\|u\|_{\sigma+2, \sigma+2}$ решения $u(t, x)$ ограничены

$$
\|u\|_{\infty, 2}+\|u\|_{\sigma+2, \sigma+2} \leqslant C .
$$

Тогда справедлива оченка

$$
\int_{0}^{t}\left\||u(\tau)|^{\sigma} \Delta u(\tau)\right\|_{\mathbf{L}^{1}} d \tau \leqslant C\langle t\rangle^{\beta}
$$

для всех $t>0$, где $\beta=0$ при $\sigma>1 u \beta>\frac{1}{\sigma}-1$ при $\frac{1}{2}<\sigma \leqslant 1$.

ДоКАЗАТЕЛЬСТвО. С помощью неравенства Гёльдера получим

$$
\|u(t)\|_{\mathbf{L}^{p}} \leqslant\|u(t)\|_{\mathbf{L}^{2}}^{1-\left(1-\frac{2}{p}\right)\left(1+\frac{2}{\sigma}\right)}\|u(t)\|_{\mathbf{L}^{2+\sigma}}^{\left(1-\frac{2}{p}\right)\left(1+\frac{2}{\sigma}\right)}
$$

при $2 \leqslant p \leqslant \sigma+2$, откуда

$$
\|u\|_{s, p} \leqslant\|u\|_{\infty, 2}^{1-\left(1-\frac{2}{p}\right)\left(1+\frac{2}{\sigma}\right)}\|u\|_{\sigma+2, \sigma+2}^{\left(1-\frac{2}{p}\right)\left(1+\frac{2}{\sigma}\right)} \leqslant C
$$

где $s=\frac{\sigma p}{p-2}$. Теперь оценим $\mathbf{L}^{p}$-норму функции $u(t)$ при $\sigma+2 \leqslant p \leqslant \infty$. Запишем (1) в виде интегрального уравнения

$$
u(t)=\mathscr{G}(t) u_{0}+\lambda \int_{0}^{t} \mathscr{G}(t-\tau) \mathscr{B}|u(\tau)|^{\sigma} u(\tau) d \tau,
$$

откуда следует неравенство

$$
\|u(t)\|_{\mathbf{L}^{p}} \leqslant\left\|\mathscr{G}(t) u_{0}\right\|_{\mathbf{L}^{p}}+|\lambda|\left\|\int_{0}^{t} \mathscr{G}(t-\tau) \mathscr{B}|u(\tau)|^{\sigma} u(\tau) d \tau\right\|_{\mathbf{L}^{p}} .
$$

В силу леммы 1 имеем оценку

$$
\begin{aligned}
\left\|\mathscr{G}(t-\tau) \mathscr{B}|u(\tau)|^{\sigma} u(\tau)\right\|_{\mathbf{L}^{p}} \leqslant & e^{-a(t-\tau)}\left\|\mathscr{B}|u(\tau)|^{\sigma} u(\tau)\right\|_{\mathbf{L}^{p}} \\
& +C\langle t-\tau\rangle^{-\frac{n}{2}\left(\frac{1}{r}-\frac{1}{p}\right)}\left\|\mathscr{B}|u(\tau)|^{\sigma} u(\tau)\right\|_{\mathbf{L}^{r}}
\end{aligned}
$$


при $1 \leqslant r \leqslant p$. Применяя теорему вложения Соболева (см. [5], [42]), получим

$$
\left\|\mathscr{B}|u(\tau)|^{\sigma} u(\tau)\right\|_{\mathbf{L}^{p}} \leqslant C\left\||u(\tau)|^{\sigma} u(\tau)\right\|_{\mathbf{L}^{r}} \leqslant C\|u(\tau)\|_{\mathbf{L}^{(\sigma+1) r}}^{\sigma+1}
$$

при $\max \left(1, \frac{p}{1+\frac{2}{n} p}\right) \leqslant r \leqslant p$. Поэтому

$$
\left\|\mathscr{G}(t-\tau) \mathscr{B}|u(\tau)|^{\sigma} u(\tau)\right\|_{\mathbf{L}^{p}} \leqslant C\langle t-\tau\rangle^{-\frac{n}{2}\left(\frac{1}{r}-\frac{1}{p}\right)}\|u(\tau)\|_{\mathbf{L}^{(\sigma+1) r}}^{\sigma+1},
$$

откуда с помошью неравенства Юнга найдем

$$
\begin{aligned}
& \left\|\int_{0}^{t}\right\| \mathscr{G}(t-\tau) \mathscr{B}|u(\tau)|^{\sigma} u(\tau)\left\|_{\mathbf{L}^{p}} d \tau\right\|_{\mathbf{L}_{t}^{s}(0, \infty)} \\
& \quad \leqslant C\left\|\int_{0}^{t}\langle t-\tau\rangle^{-\frac{n}{2}\left(\frac{1}{r}-\frac{1}{p}\right)}\right\| u(\tau)\left\|_{\mathbf{L}^{(\sigma+1) r}}^{\sigma+1} d \tau\right\|_{\mathbf{L}_{t}^{s}(0, \infty)} \\
& \quad \leqslant C\left\|\langle t\rangle^{-\frac{n}{2}\left(\frac{1}{r}-\frac{1}{p}\right)}\right\|_{\mathbf{L}_{t}^{q^{\prime}}(0, \infty)}\|u\|_{(\sigma+1) q,(\sigma+1) r}^{\sigma+1} \\
& \quad \leqslant C\|u\|_{(\sigma+1) q,(\sigma+1) r}^{\sigma+1}
\end{aligned}
$$

при условии, что $\frac{n}{2}\left(\frac{1}{r}-\frac{1}{p}\right)>\frac{1}{q^{\prime}}=\frac{1}{s}-\frac{1}{q}+1$. Воспользуемся этим неравенством, взяв $q=r=\frac{\sigma+2}{\sigma+1}, n=1,2$, следовательно, $s>\max \left(1,\left(\frac{\sigma+1}{\sigma+2}\left(1+\frac{n}{2}\right)-1-\frac{n}{2 p}\right)^{-1}\right)$. Таким образом, при $p=\infty$ получаем оценку

$$
\|u\|_{s, \infty} \leqslant C
$$

для всех $s>\frac{2(\sigma+2)}{n(\sigma+1)-2}$.

Теперь оценим $\Delta u$. Отметим, что из-за сглаживающего свойства оператора $\mathscr{B}$, стоящего перед нелинейностью в интегральном уравнении (10), нетрудно установить, что решение $u(t) \in \mathbf{W}_{\infty}^{2}\left(\mathbb{R}^{n}\right) \cap \mathbf{W}_{1}^{2}\left(\mathbb{R}^{n}\right)$ при всех $t \geqslant 0$, когда начальные данные $u_{0} \in \mathbf{W}_{\infty}^{2}\left(\mathbb{R}^{n}\right) \cap \mathbf{W}_{1}^{2}\left(\mathbb{R}^{n}\right)$. В силу второй оценки леммы 1 имеем

$$
\left\|\Delta \mathscr{G}(t-\tau) \mathscr{B}|u(\tau)|^{\sigma} u(\tau)\right\|_{\mathbf{L}^{p}} \leqslant C\langle t-\tau\rangle^{-1}\|u(\tau)\|_{\mathbf{L}^{(\sigma+1) p}}^{\sigma+1}
$$

при $p \geqslant 1$, поэтому

$$
\begin{aligned}
& \left\|\int_{0}^{t}\right\| \Delta \mathscr{G}(t-\tau) \mathscr{B}|u(\tau)|^{\sigma} u(\tau)\left\|_{\mathbf{L}^{p}} d \tau\right\|_{\mathbf{L}_{t}^{s}(0, \infty)} \\
& \leqslant C\left\|\int_{0}^{t}\langle t-\tau\rangle^{-1}\right\| u(\tau)\left\|_{\mathbf{L}^{(\sigma+1) p}}^{\sigma+1} d \tau\right\|_{\mathbf{L}_{t}^{s}(0, \infty)} \\
& \leqslant C\left\|\langle t\rangle^{-1}\right\|_{\mathbf{L}_{t}^{q^{\prime}(0, \infty)}}\|u\|_{(\sigma+1) q,(\sigma+1) p}^{\sigma+1} \\
& \leqslant C\|u\|_{(\sigma+1) q,(\sigma+1) p}^{\sigma+1}
\end{aligned}
$$

при $\frac{1}{s}=\frac{1}{q}+\frac{1}{q^{\prime}}-1, q^{\prime}>1$. Таким образом, получаем

$$
\|\Delta u\|_{s, p} \leqslant C\|u\|_{(\sigma+1) q,(\sigma+1) p}^{\sigma+1}
$$


при $s>q$. Используя неравенство

$$
\|u(\tau)\|_{\mathbf{L}^{\sigma(\sigma+2)}}^{\sigma} \leqslant\|u(\tau)\|_{\mathbf{L}^{\infty}}^{\sigma-1}\|u(\tau)\|_{\mathbf{L}^{\sigma+2}}
$$

с помощью неравенства Гёльдера находим

$$
\begin{aligned}
& \int_{0}^{t}\left\||u(\tau)|^{\sigma} \Delta u(\tau)\right\|_{\mathbf{L}^{1}} d \tau \leqslant \int_{0}^{t}\|u(\tau)\|_{\mathbf{L}^{\sigma(\sigma+2)}}^{\sigma}\|\Delta u(\tau)\|_{\mathbf{L}^{\frac{\sigma+2}{\sigma+1}}} d \tau \\
& \leqslant t^{1-\frac{1}{s_{1}}-\frac{1}{s_{2}}-\frac{1}{\sigma+2}}\|u(\tau)\|_{s_{1}(\sigma-1), \infty}^{\sigma-1}\|u(\tau)\|_{\sigma+2, \sigma+2}\|u\|_{(\sigma+1) s_{3}, \sigma+2}^{\sigma+1} \leqslant C
\end{aligned}
$$

при $\sigma>1$, поскольку мы можем выбрать $s_{1}(\sigma-1)>\frac{2(\sigma+2)}{n(\sigma+1)-2}, s_{2}>s_{3}=\frac{\sigma+2}{\sigma+1}$, так что $\frac{1}{s_{1}}+\frac{1}{s_{2}}+\frac{1}{\sigma+2}>1$. Рассмотрим теперь $\sqrt{3}-1<\sigma \leqslant 1$; тогда

$$
\begin{aligned}
& \int_{0}^{t}\left\||u(\tau)|^{\sigma} \Delta u(\tau)\right\|_{\mathbf{L}^{1}} d \tau \leqslant \int_{0}^{t}\|u(\tau)\|_{\mathbf{L}^{\sigma(\sigma+2)}}^{\sigma}\|\Delta u(\tau)\|_{\mathbf{L}^{\frac{\sigma+2}{\sigma+1}}} d \tau \\
& \quad \leqslant C\langle t\rangle^{1-\frac{1}{s_{1}}-\frac{1}{s_{2}}\|u(\tau)\|_{s_{1} \sigma, \sigma(\sigma+2)}^{\sigma}\|u\|_{(\sigma+1) s_{3}, \sigma+2}^{\sigma+1} \leqslant C\langle t\rangle^{\beta}}
\end{aligned}
$$

где мы берем $s_{1} \sigma>\frac{\sigma^{2}(\sigma+2)}{\sigma(\sigma+2)-2}, s_{2}>s_{3}=\frac{\sigma+2}{\sigma+1}$, так что $\beta=1-\frac{1}{s_{1}}-\frac{1}{s_{2}}>\frac{1}{\sigma}-1$. Наконец, при $\frac{1}{2}<\sigma \leqslant \sqrt{3}-1$ получим

$$
\begin{aligned}
\int_{0}^{t}\left\||u(\tau)|^{\sigma} \Delta u(\tau)\right\|_{\mathbf{L}^{1}} d \tau & \leqslant \int_{0}^{t}\|u(\tau)\|_{\mathbf{L}^{2}}^{\sigma}\|\Delta u(\tau)\|_{\mathbf{L}^{\frac{2}{2-\sigma}}} d \tau \\
& \leqslant C\langle t\rangle^{1-\frac{1}{s_{1}}\|u(\tau)\|_{\infty, 2}^{\sigma}\|u\|_{(\sigma+1) s_{2},(\sigma+1) \frac{2}{2-\sigma}}^{\sigma+1} \leqslant C\langle t\rangle^{\beta}}
\end{aligned}
$$

поскольку можно взять $s_{1}>s_{2}=\frac{\sigma}{2 \sigma-1}$, так что $\beta=1-\frac{1}{s_{1}}>\frac{1}{\sigma}-1$. Лемма доказана.

Следуюшая лемма необходима для того, чтобы уточнить оценку леммы 3 для случая $\sigma \leqslant 1$.

ЛЕмма 4. Пусть $n=2, \sigma \in\left(\frac{3}{4}, 1\right]$. Предположсим, что начальные данные $u_{0} \in \mathbf{W}_{\infty}^{2}\left(\mathbb{R}^{2}\right) \cap \mathbf{W}_{1}^{2}\left(\mathbb{R}^{2}\right)$. Допустим также, что выполнена оценка решения

$$
\|u(t)\|_{\mathbf{L}^{p}} \leqslant C\langle t\rangle^{\alpha-1+\frac{1}{p}}
$$

для всех $t>0$, где $1 \leqslant p \leqslant \sigma+2, \alpha \in\left[0, \frac{1}{2}\right)$. Тогда справедлива оченка

$$
\int_{0}^{t}\left\||u(\tau)|^{\sigma} \Delta u(\tau)\right\|_{\mathbf{L}^{1}} d \tau \leqslant C\langle t\rangle^{\beta}
$$

для всех $t>0$, где $\beta=0$, когда $\max \left(\sigma \alpha+\frac{1}{\sigma+2}-\sigma, \alpha(1+2 \sigma)+1-2 \sigma\right)<0$, и $\beta>\max \left(\sigma \alpha+\frac{1}{\sigma+2}-\sigma, \alpha(1+2 \sigma)+1-2 \sigma\right)$ в противном случае. 
ДоКАЗАТЕЛЬСТВо. Из оценки (21) получим

$$
\begin{aligned}
\|\Delta u(t)\|_{\mathbf{L}^{\frac{\sigma+2}{\sigma+1}}} & \leqslant C+\int_{0}^{t}\left\|\Delta \mathscr{G}(t-\tau) \mathscr{B}|u|^{\sigma} u(\tau)\right\|_{\mathbf{L}^{\frac{\sigma+2}{\sigma+1}}} d \tau \\
& \leqslant C \int_{0}^{t}\langle t-\tau\rangle^{-1}\|u(\tau)\|_{\mathbf{L}^{\sigma+2}}^{\sigma+1} d \tau \\
& \leqslant C \int_{0}^{t}\langle t-\tau\rangle^{-1}\langle\tau\rangle^{(\sigma+1)\left(\alpha-\frac{\sigma+1}{\sigma+2}\right)} d \tau \leqslant C\langle t\rangle^{\delta}
\end{aligned}
$$

для всех $t>0$, где $\delta>\max \left(-1,(\sigma+1)\left(\alpha-\frac{\sigma+1}{\sigma+2}\right)\right)$. Тогда

$$
\begin{aligned}
\int_{0}^{t}\left\||u(\tau)|^{\sigma} \Delta u(\tau)\right\|_{\mathbf{L}^{1}} d \tau & \leqslant \int_{0}^{t}\|u(\tau)\|_{\mathbf{L}^{\sigma(\sigma+2)}}^{\sigma}\|\Delta u(\tau)\|_{\mathbf{L}^{\frac{\sigma+2}{\sigma+1}}} d \tau \\
& \leqslant \int_{0}^{t}\langle\tau\rangle^{\sigma(\alpha-1)+\frac{1}{\sigma+2}}\langle\tau\rangle^{\delta} d \tau \leqslant C\langle t\rangle^{\beta}
\end{aligned}
$$

где

$$
\beta=0, \quad \text { если } \max \left(\sigma \alpha+\frac{1}{\sigma+2}-\sigma, \alpha(1+2 \sigma)+1-2 \sigma\right)<0,
$$

$$
\beta>\max \left(\sigma \alpha+\frac{1}{\sigma+2}-\sigma, \alpha(1+2 \sigma)+1-2 \sigma\right)
$$

в противном случае. Лемма доказана.

В следующей лемме мы установим зависимость от времени $\mathbf{L}^{p}$-нормы решения.

Лемма 5. Пусть $u_{0} \in \mathbf{H}^{2}\left(\mathbb{R}^{n}\right) \cap \mathbf{L}^{1}\left(\mathbb{R}^{n}\right)$. Предположим, что

$$
\int_{0}^{t} d \tau|||u(\tau)|^{\sigma} \Delta u(\tau) \|_{\mathbf{L}^{1}} \leqslant C\langle t\rangle^{\beta}
$$

для всех $t>0$, где $\beta \in\left[0, \frac{n}{4}\right)$. Тогда выполнена оценка

$$
\|u(t)\|_{\mathbf{L}^{p}} \leqslant C\langle t\rangle^{\beta-\frac{n}{2}\left(1-\frac{1}{p}\right)}
$$

для всех $t>0$ nрu $1 \leqslant p \leqslant \sigma+2$.

ДоКАЗАТЕЛЬСТво. Заменим $w=(1-\Delta) u$; тогда из (1) получим уравнение

$$
\partial_{t} w=\alpha(\mathscr{B}-1) w+\lambda|\mathscr{B} w|^{\sigma} w+\lambda|u|^{\sigma} \Delta u,
$$

где $\mathscr{B}=(1-\Delta)^{-1}$. Оценим сначала $\mathbf{L}^{1}$-норму. Пусть $\Theta(x)=1$ при $x>0$ и $\Theta(x)=-1$ при $x<0 ; \Theta(0)=0$. Уравнение (23) умножим на $\Theta(w(t, x))$ и проинтегрируем по $x$ по всему пространству $\mathbb{R}^{n}$. Тогда

$$
\begin{aligned}
& \int_{\mathbb{R}^{n}} \partial_{t} w(t, x) \Theta(w(t, x)) d x=\alpha \int_{\mathbb{R}^{n}} \Theta(w(t, x))(\mathscr{B}-1) w d x \\
& \quad+\lambda \int_{\mathbb{R}^{n}}|\mathscr{B} w(t, x)|^{\sigma}|w(t, x)| d x+\lambda \int_{\mathbb{R}^{n}} \Theta(w(t, x))|u(t, x)|^{\sigma} \Delta u(t, x) d x .
\end{aligned}
$$


Имеем

$$
\begin{gathered}
\int_{\mathbb{R}^{n}} w_{t}(t, x) \Theta(w(t, x)) d x=\int_{\mathbb{R}^{n}} \frac{\partial}{\partial t}|w(t, x)| d x=\frac{d}{d t}\|w(t)\|_{\mathbf{L}^{1}}, \\
\lambda \int_{\mathbb{R}^{n}}|\mathscr{B} w(t, x)|^{\sigma}|w(t, x)| d x \leqslant 0 \\
\int_{\mathbb{R}^{n}} \Theta(w(t, x)) \mathscr{B} w d x \leqslant\|\mathscr{B} w(t)\|_{\mathbf{L}^{1}} \leqslant\|w(t)\|_{\mathbf{L}^{1}},
\end{gathered}
$$

так что

$$
\begin{aligned}
& \alpha \int_{\mathbb{R}^{n}} \Theta(w(t, x))(\mathscr{B}-1) w d x \\
& \quad=\alpha\left(\int_{\mathbb{R}^{n}} \Theta(w(t, x)) \mathscr{B} w d x-\|w(t)\|_{\mathbf{L}^{1}}\right) \leqslant 0
\end{aligned}
$$

откуда находим

$$
\frac{d}{d t}\|w(t)\|_{\mathbf{L}^{1}} \leqslant|\lambda|\left\||u(t)|^{\sigma} \Delta u(t)\right\|_{\mathbf{L}^{1}} .
$$

Интегрируя неравенство (24), ввиду оценки (22) получаем

$$
\|w(t)\|_{\mathbf{L}^{1}} \leqslant\left\|w_{0}\right\|_{\mathbf{L}^{1}}+C\langle t\rangle^{\beta}
$$

В частности, в силу (5) имеем

$$
\sup _{\xi \in \mathbb{R}}|\widehat{u}(t, \xi)| \leqslant(2 \pi)^{-\frac{n}{2}}\|\mathscr{B} w(t)\|_{\mathbf{L}^{1}} \leqslant C\|w(t)\|_{\mathbf{L}^{1}} \leqslant C\langle t\rangle^{\beta} .
$$

Таким образом, оценка леммы при $p=1$ выполнена.

Умножим теперь уравнение (1) на $2 u$ и, проинтегрировав по $x \in \mathbb{R}^{n}$, получим

$$
\frac{d}{d t}\left(\|u(t)\|_{\mathbf{L}^{2}}^{2}+\|\nabla u(t)\|_{\mathbf{L}^{2}}^{2}\right)=-2 \alpha\|\nabla u(t)\|_{\mathbf{L}^{2}}^{2}+2 \lambda\|u(t)\|_{\mathbf{L}^{\sigma+2}}^{\sigma+2}
$$

(отметим, что из интегрального уравнения (10) легко следует, что решение $u(t) \in$ $\mathbf{H}^{2}\left(\mathbb{R}^{n}\right) \cap \mathbf{L}^{1}\left(\mathbb{R}^{n}\right)$ при всех $t \geqslant 0$ в случае, когда $u_{0} \in \mathbf{H}^{2}\left(\mathbb{R}^{n}\right) \cap \mathbf{L}^{1}\left(\mathbb{R}^{n}\right)$, поэтому интегрирование по частям здесь оправдано). С помощњю теоремы Планшереля, следуя методу работы [47], найдем

$$
\begin{aligned}
\|\nabla u(t)\|_{\mathbf{L}^{2}}^{2} & =\||\xi| \widehat{u}(t)\|_{\mathbf{L}^{2}}^{2}=\int_{|\xi| \leqslant \delta}|\widehat{u}(t, \xi)|^{2}|\xi|^{2} d \xi+\int_{|\xi| \geqslant \delta}|\widehat{u}(t, \xi)|^{2}|\xi|^{2} d \xi \\
& \geqslant \delta^{2}\|u(t)\|_{\mathbf{L}^{2}}^{2}-C \delta^{2+n} \sup _{|\xi| \leqslant \delta}|\widehat{u}(t, \xi)|^{2}
\end{aligned}
$$

где $\delta \in(0,1)$. Таким образом, из $(26)$ приходим к неравенству

$$
\frac{d}{d t}\|u(t)\|_{\mathbf{H}^{1}}^{2} \leqslant-\alpha \delta^{2}\|u(t)\|_{\mathbf{H}^{1}}^{2}+C \delta^{2+n} \sup _{|\xi| \leqslant \delta}|\widehat{u}(t, \xi)|^{2} .
$$


Выберем $\alpha \delta^{2}=(1+n)\left(t_{0}+t\right)^{-1}, t_{0}=\sqrt{\frac{1+n}{\alpha}}$, и заменим $\|u(t)\|_{\mathbf{H}^{1}}^{2}=$ $\left(t_{0}+t\right)^{-1-n} W(t)$. Тогда в силу $(25)$ получим из $(27)$

$$
\frac{d}{d t} W(t) \leqslant C\left(t_{0}+t\right)^{2 \beta+\frac{n}{2}} .
$$

Интегрируя (28) по времени, имеем

$$
W(t) \leqslant\left\|u_{0}\right\|_{\mathbf{H}^{1}}^{2}+C\left(\left(t_{0}+t\right)^{\frac{n}{2}+1+2 \beta}-1\right),
$$

откуда получаем оценку убывания по времени $\mathbf{L}^{2}$-нормы решения

$$
\|u(t)\|_{\mathbf{L}^{2}} \leqslant C(1+t)^{\beta-\frac{n}{4}}
$$

для всех $t>0$.

Аналогично, умножив уравнение (1) на $4 u^{3}$ и проинтегрировав по $x \in \mathbb{R}^{n}$, получим

$$
\frac{d}{d t}\left\|u^{2}(t)\right\|_{\mathbf{H}^{1}}^{2}=-3 \alpha\left\|\nabla\left(u^{2}(t)\right)\right\|_{\mathbf{L}^{2}}^{2}+4 \lambda\|u(t)\|_{\mathbf{L}^{\sigma+4}}^{\sigma+4} .
$$

Как и вьшее, учитывая (29), имеем

$$
\begin{aligned}
\left\|\nabla\left(u^{2}(t)\right)\right\|_{\mathbf{L}^{2}}^{2} & =\left\|\mid \widehat{\mid u^{2}}(t)\right\|_{\mathbf{L}^{2}}^{2} \\
& =\int_{|\xi| \leqslant \delta}\left|\widehat{u^{2}}(t, \xi)\right|^{2}|\xi|^{2} d \xi+\int_{|\xi| \geqslant \delta}\left|\widehat{u^{2}}(t, \xi)\right|^{2}|\xi|^{2} d \xi \\
& \geqslant \delta^{2}\|u(t)\|_{\mathbf{L}^{4}}^{4}-C \delta^{2+n} \sup _{|\xi| \leqslant \delta}\left|\widehat{u^{2}}(t, \xi)\right|^{2} \\
& \geqslant \delta^{2}\|u(t)\|_{\mathbf{L}^{4}}^{4}-C \delta^{2+n}\|u(t)\|_{\mathbf{L}^{2}}^{4},
\end{aligned}
$$

где $\delta>0$. Таким образом, из (30) приходим к неравенству

$$
\frac{d}{d t}\left\|u^{2}(t)\right\|_{\mathbf{H}^{1}}^{2} \leqslant-\alpha \delta^{2}\left\|u^{2}(t)\right\|_{\mathbf{H}^{1}}^{2}+C \delta^{2+n}(1+t)^{4 \beta-n} .
$$

Выберем на этот раз $\alpha \delta^{2}=(1+2 n)\left(t_{1}+t\right)^{-1}, t_{1}=\sqrt{\frac{1+2 n}{\alpha}}$, и заменим

$$
\left\|u^{2}(t)\right\|_{\mathbf{H}^{1}}^{2}=\left(t_{1}+t\right)^{-1-2 n} W_{1}(t)
$$

Тогда из (31) найдем

$$
\frac{d}{d t} W_{1}(t) \leqslant C\left(t_{1}+t\right)^{4 \beta+\frac{n}{2}} .
$$

Интегрируя (32) по времени, получаем

$$
W_{1}(t) \leqslant\left\|u_{0}^{2}\right\|_{\mathbf{H}^{1}}^{2}+C\left(\left(t_{1}+t\right)^{\frac{n}{2}+1+4 \beta}-1\right),
$$


откуда следует оценка убывания по времени $\mathbf{L}^{4}$-нормы решения

$$
\|u(t)\|_{\mathbf{L}^{4}} \leqslant C(1+t)^{\beta-\frac{3 n}{8}}
$$

для всех $t>0$. Продолжая эти рассуждения и используя неравенство Гёльдера, ввиду (25) и (33) приходим к оценке леммы. Лемма доказана.

УТВЕРЖДЕНИЕ 2. Пусть $\sigma>1$ nрu $n=1 u \sigma>\frac{3}{4}$ nрu $n=2$. Предположим, что начальные данные $u_{0} \in \mathbf{W}_{\infty}^{2}\left(\mathbb{R}^{n}\right) \cap \mathbf{W}_{1}^{2}\left(\mathbb{R}^{n}\right)$. Тогда справедлива оченка

$$
\|u(t)\|_{\mathbf{L}^{p}} \leqslant C\langle t\rangle^{-\frac{n}{2}\left(1-\frac{1}{p}\right)}
$$

для всех $t>0$ при $1 \leqslant p \leqslant \sigma+2$.

ДокАЗАТЕЛЬСтво. Интегрируя формулу (26) по времени, имеем

$$
\begin{aligned}
& \|u(t)\|_{\mathbf{L}^{2}}^{2}+\|\nabla u(t)\|_{\mathbf{L}^{2}}^{2}+2 \alpha \int_{0}^{t}\|\nabla u(\tau)\|_{\mathbf{L}^{2}}^{2} d \tau-2 \lambda \int_{0}^{t}\|u(\tau)\|_{\mathbf{L}^{\sigma+2}}^{\sigma+2} d \tau \\
& =\left\|u_{0}\right\|_{\mathbf{L}^{2}}^{2}+\left\|\nabla u_{0}\right\|_{\mathbf{L}^{2}}^{2}=\left\|u_{0}\right\|_{\mathbf{H}^{1}}^{2}
\end{aligned}
$$

для всех $t \geqslant 0$. В частности, имеем оценки

$$
\begin{gathered}
\|u\|_{\infty, 2} \equiv \sup _{t \geqslant 0}\|u(t)\|_{\mathbf{L}^{2}} \leqslant\left\|u_{0}\right\|_{\mathbf{H}^{1}}, \\
\|u\|_{\sigma+2, \sigma+2} \equiv\|\| u(t, x)\left\|_{\mathbf{L}_{x}^{\sigma+2}}\right\|_{\mathbf{L}_{t}^{\sigma+2}(0, \infty)} \leqslant C\left\|u_{0}\right\|_{\mathbf{H}^{1}} .
\end{gathered}
$$

В силу (34) и (35), применяя лемму 3 , получаем

$$
\int_{0}^{t} d \tau\left\||u(\tau)|^{\sigma} \Delta u(\tau)\right\|_{\mathbf{L}^{1}} \leqslant C
$$

для всех $t>0$ при $\sigma>1$. Таким образом, результат утверждения в случае $\sigma>1$ следует из леммы 5. Рассмотрим случай $\sigma \in\left(\frac{3}{4}, 1\right]$, когда $n=2$. Из леммы 3 имеем

$$
\int_{0}^{t} d \tau\left\||u(\tau)|^{\sigma} \Delta u(\tau)\right\|_{\mathbf{L}^{1}} \leqslant C\langle t\rangle^{\beta_{1}}
$$

для всех $t>0$, где $\beta_{1}>\frac{1}{\sigma}-1$. Тогда в силу леммы 5 находим

$$
\|u(t)\|_{\mathbf{L}^{p}} \leqslant C\langle t\rangle^{\beta_{1}-\frac{n}{2}\left(1-\frac{1}{p}\right)}
$$

для всех $t>0$ при $1 \leqslant p \leqslant \sigma+2$. Теперь с помощью леммы 4 получаем

$$
\int_{0}^{t}\left\||u(\tau)|^{\sigma} \Delta u(\tau)\right\|_{\mathbf{L}^{1}} d \tau \leqslant C\langle t\rangle^{\beta_{2}}
$$

для всех $t>0$, где $\beta_{2}=0$, если $\sigma \in\left(\frac{1}{4}(1+\sqrt{5}), 1\right]$, и $\beta_{2}>\frac{1}{\sigma}-4 \sigma+2$ в противном случае. 
Снова применив лемму 5 , найдем

$$
\|u(t)\|_{\mathbf{L}^{p}} \leqslant C\langle t\rangle^{\beta_{2}-\frac{n}{2}\left(1-\frac{1}{p}\right)}
$$

для всех $t>0$ при $1 \leqslant p \leqslant \sigma+2$. После этого, используя лемму 4 , получим

$$
\int_{0}^{t}\left\||u(\tau)|^{\sigma} \Delta u(\tau)\right\|_{\mathbf{L}^{1}} d \tau \leqslant C\langle t\rangle^{\beta_{3}}
$$

для всех $t>0$, где $\beta_{3}=0$ при $\sigma>0.775$ и $\beta_{3}>\beta_{2}(1+2 \sigma)+1-2 \sigma$ в противном случае. Продолжив эти рассуждения, получим окончательно

$$
\int_{0}^{t}|||u(\tau)|^{\sigma} \Delta u(\tau) \|_{\mathbf{L}^{1}} d \tau \leqslant C
$$

для всех $t>0$, если $\sigma>\frac{3}{4}$. Откуда в силу леммы 5 приходим к оценке убывания по времени

$$
\|u(t)\|_{\mathbf{L}^{p}} \leqslant C\langle t\rangle^{-\frac{n}{2}\left(1-\frac{1}{p}\right)}
$$

для всех $t>0$ при $1 \leqslant p \leqslant \sigma+2$, если $\sigma \in\left(\frac{3}{4}, 1\right]$, и $n=2$. Утверждение доказано.

ДОКАЗАТЕЛЬСТво ТЕОРЕМЫ 2. Используя утверждение 2, мы получаем следующую точную оценку убывания по времени решения:

$$
\|u(t)\|_{\mathbf{L}^{p}} \leqslant C\langle t\rangle^{-\frac{n}{2}\left(1-\frac{1}{p}\right)}
$$

для всех $t>0$ при $1 \leqslant p \leqslant \sigma+1$. Докажем (37) также и для $p=\infty$. Достаточно рассмотреть $t \geqslant T>0$, поскольку при $t \in[0, T]$ оценка (37) следует из утверждения 1. Применяя первую оценку леммы 1 с $p=\infty$ и $r=\frac{\sigma+2}{\sigma+1}$, ввиду (5) и (19) имеем

$$
\begin{aligned}
& \left\|\mathscr{G}(t-\tau) \mathscr{B}|u|^{\sigma} u(\tau)\right\|_{\mathbf{L}^{\infty}} \\
& \quad \leqslant C e^{-a t}\left\|\mathscr{B}|u|^{\sigma} u(\tau)\right\|_{\mathbf{L}^{\infty}}+C(t-\tau)^{-\frac{n(\sigma+1)}{2(\sigma+2)}}\left\|\mathscr{B}|u|^{\sigma} u(\tau)\right\|_{\mathbf{L}^{\frac{\sigma+2}{\sigma+1}}} \\
& \quad \leqslant C(t-\tau)^{-\frac{n(\sigma+1)}{2(\sigma+2)}}\|u(\tau)\|_{\mathbf{L}^{\sigma+2}}^{\sigma+1}
\end{aligned}
$$

в области $\frac{t}{2} \leqslant \tau \leqslant t$. В области $0 \leqslant \tau \leqslant \frac{t}{2}$ с помощью первой оценки леммы 1 при $p=\infty$ и $r=1$ получаем

$$
\begin{aligned}
& \left\|\mathscr{G}(t-\tau) \mathscr{B}|u|^{\sigma} u(\tau)\right\|_{\mathbf{L}^{\infty}} \\
& \quad \leqslant C e^{-a t}\left\|\mathscr{B}|u|^{\sigma} u(\tau)\right\|_{\mathbf{L}^{\infty}}+C(t-\tau)^{-\frac{n}{2}}\left\|\mathscr{B}|u|^{\sigma} u(\tau)\right\|_{\mathbf{L}^{1}} \\
& \quad \leqslant C(t-\tau)^{-\frac{n}{2}}\left(\|u(\tau)\|_{\mathbf{L}^{\sigma+2}}^{\sigma+1}+\|u(\tau)\|_{\mathbf{L}^{\sigma+1}}^{\sigma+1}\right) .
\end{aligned}
$$


Поэтому из интегрального уравнения (10) находим

$$
\begin{aligned}
\|u(t)\|_{\mathbf{L}} \infty \leqslant & \left\|\mathscr{G}(t) u_{0}\right\|_{\mathbf{L}^{\infty}}+C \int_{0}^{t}\left\|\mathscr{G}(t-\tau) \mathscr{B}|u|^{\sigma} u(\tau)\right\|_{\mathbf{L}^{\infty}} d \tau \\
\leqslant & \left\|\mathscr{G}(t) u_{0}\right\|_{\mathbf{L}}+C \int_{\frac{t}{2}}^{t}(t-\tau)^{-\frac{n(\sigma+1)}{2(\sigma+2)}}\|u(\tau)\|_{\mathbf{L}^{\sigma+2}}^{\sigma+1} d \tau \\
& +C \int_{0}^{\frac{t}{2}}(t-\tau)^{-\frac{n}{2}}\left(\|u(\tau)\|_{\mathbf{L}^{\sigma+2}}^{\sigma+1}+\|u(\tau)\|_{\mathbf{L}^{\sigma+1}}^{\sigma+1}\right) d \tau \\
\leqslant & C t^{-\frac{n}{2}}+C \int_{\frac{t}{2}}^{t}(t-\tau)^{-\frac{n(\sigma+1)}{2(\sigma+2)}}\langle\tau\rangle^{-\frac{n(\sigma+1)^{2}}{2(\sigma+2)}} d \tau \\
& +C \int_{0}^{\frac{t}{2}}(t-\tau)^{-\frac{n}{2}}\langle\tau\rangle^{-\frac{n}{2} \sigma} d \tau \leqslant C t^{-\frac{n}{2}}
\end{aligned}
$$

для всех $t \geqslant 1$, поскольку порядок нелинейности $\sigma$ суперкритический, т. е. $\frac{n}{2} \sigma>1$. Теперь (37) вытекает для всех $1 \leqslant p \leqslant \infty$ благодаря неравенству Гёльдера.

Оценим $\mathbf{L}^{1, a}$-норму решения. В силу четвертой оценки леммы 1 ввиду (5) и (19) найдем

$$
\begin{aligned}
& \left\|\mathscr{G}(t-\tau) \mathscr{B}|u|^{\sigma} u(\tau)\right\|_{\mathbf{L}^{1, a}} \\
& \quad \leqslant C\langle t-\tau\rangle^{\frac{a}{2}}\left\|\mathscr{B}|u|^{\sigma} u(\tau)\right\|_{\mathbf{L}^{1}}+C\left\|\mathscr{B}|u|^{\sigma} u(\tau)\right\|_{\mathbf{L}^{1, a}} \\
& \quad \leqslant C\langle t-\tau\rangle^{\frac{a}{2}}\|u(\tau)\|_{\mathbf{L}^{\infty}}^{\sigma}\|u(\tau)\|_{\mathbf{L}^{1}}+C\|u(\tau)\|_{\mathbf{L}^{\infty} \infty u(\tau) \|_{\mathbf{L}^{1, a}}},
\end{aligned}
$$

поэтому из интегрального уравнения (10) вытекает оценка

$$
\begin{aligned}
\|u(t)\|_{\mathbf{L}^{1, a}} \leqslant & \left\|\mathscr{G}(t) u_{0}\right\|_{\mathbf{L}^{1, a}} \\
& +C \int_{0}^{t}\langle t-\tau\rangle^{\frac{a}{2}}\langle\tau\rangle^{-\frac{n}{2} \sigma} d \tau+C \int_{0}^{t}\langle\tau\rangle^{-\frac{n}{2} \sigma}\|u(\tau)\|_{\mathbf{L}^{1, a}} d \tau \\
\leqslant & C\langle t\rangle^{\frac{a}{2}}+C \int_{0}^{t}\langle\tau\rangle^{-\frac{n}{2} \sigma}\|u(\tau)\|_{\mathbf{L}^{1, a}} d \tau,
\end{aligned}
$$

откуда с помощью леммы Гронуолла получаем

$$
\|u(t)\|_{\mathbf{L}^{1, a}} \leqslant C\langle t\rangle^{\frac{a}{2}}
$$

для всех $t>0$. Значит, мы снова приходим к точной оценке убывания по времени (15). В силу (15) локальное решение, построенное в утверждении 1 , может быть продолжено для любого $t>0$, следовательно, сушествует единственное решение $u \in \mathbf{C}\left([0, \infty) ; \mathbf{C}\left(\mathbb{R}^{n}\right) \cap \mathbf{L}^{\infty}\left(\mathbb{R}^{n}\right) \cap \mathbf{L}^{1, a}\left(\mathbb{R}^{n}\right)\right)$ задачи Коши (1). Рассуждая теперь так же, как и при доказательстве теоремы 1 , получаем асимптотику (14). Теорема доказана. 


\section{§4. Критический случай}

Настоящий параграф посвяшен исследованию задачи Коши (1) в критическом случае $\sigma=\frac{2}{n}$.

4.1. Малые начальные данные. Определим

$$
\eta=2 t \int_{\mathbb{R}^{n}}\left(G_{0}(t, x)\right)^{\frac{2}{n}+1} d x=\frac{1}{2 \pi \alpha}\left(\frac{2}{n}+1\right)^{-\frac{n}{2}},
$$

где

$$
G_{0}(t, x)=(4 \pi \alpha t)^{-\frac{n}{2}} e^{-\frac{|x|^{2}}{4 \alpha t}}
$$

Обозначим $g(t)=1+|\theta|^{\frac{2}{n}} \eta \log (1+t), \theta=\int_{\mathbb{R}^{n}} u_{0}(x) d x$. Сформулируем основной результат этого параграфа.

ТЕОРема 3. Предположим, что $\lambda<0$. Пусть начальные данные $u_{0} \in$ $\mathbf{C}\left(\mathbb{R}^{n}\right) \cap \mathbf{L}^{\infty}\left(\mathbb{R}^{n}\right) \cap \mathbf{L}^{1, a}\left(\mathbb{R}^{n}\right), \quad a \in(0,1]$, являются достаточно мальмии: $\left\|u_{0}\right\|_{\mathbf{L}^{\infty}}+\left\|u_{0}\right\|_{\mathbf{L}^{1, a}} \leqslant \varepsilon, u \theta \geqslant C \varepsilon>0$. Тогда задача Коии (1) имеет единственное глобальное во времени речение $u(t, x) \in \mathbf{C}\left([0, \infty) ; \mathbf{C}\left(\mathbb{R}^{n}\right) \cap \mathbf{L}^{\infty}\left(\mathbb{R}^{n}\right) \cap\right.$ $\left.\mathbf{L}^{1, a}\left(\mathbb{R}^{n}\right)\right)$ и для него справедлива оченка

$$
\left\|u(t)-\theta G_{0}(t) g^{-\frac{n}{2}}(t)\right\|_{\mathbf{L}^{\infty}} \leqslant C\langle t\rangle^{-\frac{n}{2}} g^{-1-\frac{n}{2}}(t) \log g(t)
$$

ДокАЗАТЕЛЬство. Как в работе [20], сделаем замену $u(t, x)=v(t, x) e^{-\varphi(t)}$. Тогда для новой функции $v(t, x)$ получаем следующее уравнение:

$$
\partial_{t}(v-\Delta v)-\alpha \Delta v-\lambda e^{-\frac{2}{n} \varphi}|v|^{\frac{2}{n}} v-\varphi^{\prime} v=0 .
$$

Функцию $\varphi(t)$ выберем из условий $\varphi(0)=0$ и

$$
\int_{\mathbb{R}^{n}}\left(\lambda e^{-\frac{2}{n} \varphi}|v|^{\frac{2}{n}} v+\varphi^{\prime} v\right) d x=0
$$

тогда среднее значение новой функции $v(t, x)$ удовлетворяет закону сохранения:

$$
\frac{d}{d t} \int_{\mathbb{R}^{n}} v(t, x) d x=0
$$

откуда

$$
\int_{\mathbb{R}^{n}} v(t, x) d x=\int_{\mathbb{R}^{n}} u_{0}(x) d x
$$

при всех $t>0$. Таким образом, мы приходим к задаче Коши для новых функций $(v(t, x), \varphi(t))$ :

$$
\begin{gathered}
\partial_{t}(v-\Delta v)-\alpha \Delta v=\lambda e^{-\frac{2}{n} \varphi}\left(|v|^{\frac{2}{n}}-\frac{1}{\theta} \int_{\mathbb{R}^{n}}|v|^{\frac{2}{n}} v d x\right) v, \\
\varphi^{\prime}(t)=-\frac{\lambda}{\theta} e^{-\frac{2}{n} \varphi} \int_{\mathbb{R}^{n}}|v|^{\frac{2}{n}} v d x \\
v(0, x)=u_{0}(x), \quad \varphi(0)=0 .
\end{gathered}
$$


Обозначим $h(t)=e^{\frac{2}{n} \varphi(t)}$ и запишем (39) в форме

$$
\begin{gathered}
\partial_{t}(v-\Delta v)-\alpha \Delta v=f(v, h), \quad v(0, x)=u_{0}(x), \\
\partial_{t} h=-\frac{2 \lambda}{\theta n} \int_{\mathbb{R}^{n}}|v|^{\frac{2}{n}} v d x, \quad h(0)=1,
\end{gathered}
$$

где

$$
f(v, h)=\lambda h^{-1}\left(|v|^{\frac{2}{n}}-\frac{1}{\theta} \int_{\mathbb{R}^{n}}|v|^{\frac{2}{n}} v d x\right) v .
$$

Заметим, что среднее значение нелинейности

$$
\int_{\mathbb{R}^{n}} f(v, h)(t, x) d x=0
$$

для всех $t>0$. Докажем сушествование решения $(v(t, x), h(t))$ задачи Коши $(40)$ с помощью метода последовательных приближений. Обозначим

$$
v_{1}=\mathscr{G}(t) u_{0}, \quad h_{1}=g(t), \quad g(t)=1+|\theta|^{\frac{2}{n}} \eta \log \langle t\rangle
$$

а функции $\left(v_{m}(t, x), h_{m}(t)\right)$ определим для всех $m \geqslant 2$ как решение следуюшей задачи (которое понимается в смысле соответствующего интегрального уравнения, получаемого ниже с помощью преобразования Фурье):

$$
\begin{gathered}
\partial_{t}\left(v_{m}-\Delta v_{m}\right)-\alpha \Delta v_{m}=f\left(v_{m-1}, h_{m-1}\right), \\
\partial_{t} h_{m}=-\frac{2 \lambda}{\theta n} \int_{\mathbb{R}^{n}}\left|v_{m-1}\right|^{\frac{2}{n}} v_{m-1} d x, \\
v_{m}(0, x)=u_{0}(x), \quad h_{m}(0)=1 .
\end{gathered}
$$

Докажем по индукции оценки

$$
\begin{gathered}
\left\|v_{m}\right\|_{\mathbf{x}} \leqslant C \varepsilon, \quad\left\|v_{m}(t)-\mathscr{G}(t) u_{0}\right\|_{\mathbf{L}^{1}} \leqslant C \varepsilon^{1+\frac{2}{n}} g^{-1}(t) \\
\left|h_{m}(t)-g(t)\right| \leqslant C \varepsilon^{\frac{2}{n}}(1+\log g(t))
\end{gathered}
$$

для всех $m \geqslant 1$, где норма $\|\cdot\| \mathbf{x}$ определена, как и вьше, следуюшим образом:

$$
\|\varphi\|_{\mathbf{X}}=\sup _{t>0}\left(\langle t\rangle^{\frac{n}{2}}\|\varphi(t)\|_{\mathbf{L}^{\infty}}+\langle t\rangle^{-\frac{a}{2}}\|\varphi(t)\|_{\mathbf{L}^{1, a}}\right) .
$$

Из леммы 1 найдем

$$
\begin{gathered}
\left\|\mathscr{G}(t) u_{0}\right\|_{\mathbf{L}^{\infty}} \leqslant C \varepsilon\langle t\rangle^{-\frac{n}{2}}, \quad\left\|\mathscr{G}(t) u_{0}\right\|_{\mathbf{L}^{1}} \leqslant C \varepsilon, \\
\left\|\left.\cdot \cdot\right|^{a}\left(\mathscr{G}(t) u_{0}-\theta G_{0}(t, x)\right)\right\|_{\mathbf{L}^{1}} \leqslant C \varepsilon
\end{gathered}
$$

и

$$
\left\||\cdot|{ }^{a} G_{0}(t, x)\right\|_{\mathbf{L}^{1}} \leqslant C t^{\frac{a}{2}} .
$$


Следовательно, оценки (42) выполнены при $m=1$. Предположим, что оценки (42) справедливы для некоторого номера $m-1$. Перепишем задачу (41) в виде интегральных уравнений

$$
\begin{gathered}
v_{m}(t)=\mathscr{G}(t) u_{0}+\int_{0}^{t} \mathscr{G}(t-\tau) \mathscr{B} f\left(v_{m-1}(\tau), h_{m-1}(\tau)\right) d \tau \\
h_{m}(t)=1-\frac{2 \lambda}{\theta n} \int_{0}^{t} d \tau \int_{\mathbb{R}^{n}}\left|v_{m-1}\right|^{\frac{2}{n}} v_{m-1} d x
\end{gathered}
$$

Имеем

$$
\begin{aligned}
& \left\|f\left(v_{m-1}(t), h_{m-1}(t)\right)\right\|_{\mathbf{L}^{\infty}} \\
& \quad \leqslant C h_{m-1}^{-1}(t)\left\|v_{m-1}(t)\right\|_{\mathbf{L}^{\infty}}^{1+\frac{2}{n}}\left(1+\frac{1}{|\theta|}\left\|v_{m-1}(t)\right\|_{\mathbf{L}^{1}}\right) \\
& \quad \leqslant C \varepsilon^{1+\frac{2}{n}}\langle t\rangle^{-1-\frac{n}{2}} g^{-1}(t), \\
& \quad\left\|f\left(v_{m-1}(t), h_{m-1}(t)\right)\right\|_{\mathbf{L}^{1, a}} \\
& \quad \leqslant C h_{m-1}^{-1}(t)\left\|v_{m-1}(t)\right\|_{\mathbf{L}^{\infty}}^{\frac{2}{n}}\left\|v_{m-1}(t)\right\|_{\mathbf{L}^{1, a}}\left(1+\frac{1}{|\theta|}\left\|v_{m-1}(t)\right\|_{\mathbf{L}^{1}}\right) \\
& \quad \leqslant C \varepsilon^{1+\frac{2}{n}}\langle t\rangle^{-1+\frac{a}{2}} g^{-1}(t)
\end{aligned}
$$

для всех $t>0$ при условии, что $\left(v_{m-1}(t), h_{m-1}(t)\right)$ подчиняется (42). Отсюда следует оценка

$$
\left\|\langle t\rangle g(t) f\left(v_{m-1}(t), h_{m-1}(t)\right)\right\|_{\mathbf{X}} \leqslant C \varepsilon^{1+\frac{2}{n}} .
$$

Поскольку $f\left(v_{m-1}(\tau), h_{m-1}(\tau)\right)$ имеет нулевое среднее значение, из леммы 2 получаем

$$
\left\|g(t) \int_{0}^{t} g^{-1}(\tau) \mathscr{G}(t-\tau) \mathscr{B} f\left(v_{m-1}(\tau), h_{m-1}(\tau)\right) d \tau\right\|_{\mathbf{X}} \leqslant C \varepsilon^{1+\frac{2}{n}},
$$

откуда следует, что

$$
\left\|v_{m}\right\|_{\mathbf{x}} \leqslant C \varepsilon, \quad\left\|v_{m}(t)-\mathscr{G}(t) u_{0}\right\|_{\mathbf{L}^{1}} \leqslant C \varepsilon^{1+\frac{2}{n}} g^{-1}(t)
$$

Чтобы доказать третью оценку в (42), нам потребуется следующая лемма, в которой мы вычислим эволюцию во времени среднего значения нелинейности в уравнении (1) в критическом случае. Как и выше, положим $\theta=\int_{\mathbb{R}^{n}} u_{0}(x) d x$.

Лемма 6. Пусть $\lambda<0$. Предположим, ито $u_{0} \in \mathbf{L}^{\infty}\left(\mathbb{R}^{n}\right) \cap \mathbf{L}^{1, a}\left(\mathbb{R}^{n}\right)$, причем норма $\left\|u_{0}\right\|_{\mathbf{L}^{\infty}}+\left\|u_{0}\right\|_{\mathbf{L}^{1, a}}=\varepsilon$ достаточно мала. Кроме того, пусть $\theta \geqslant C \varepsilon>0$. Предположим,что функиия $v(t, x)$ подчиняется оценкам

$$
\begin{gathered}
\langle t\rangle^{\frac{n}{2}}\|v\|_{\mathbf{L}^{\infty}}+\|v\|_{\mathbf{L}^{1}} \leqslant C \varepsilon, \\
\left\|v(t)-\mathscr{G}(t) u_{0}\right\|_{\mathbf{L}^{1}} \leqslant C \varepsilon^{1+\frac{2}{n}} g^{-1}(t)
\end{gathered}
$$

для всех $t>0$. Тогда имеет место неравенство

$$
\left.\left|1-\frac{2 \lambda}{\theta n} \int_{0}^{t} d \tau \int_{\mathbb{R}^{n}}\right| v\right|^{\frac{2}{n}} v(\tau, x) d x-g(t) \mid \leqslant C \varepsilon^{\frac{2}{n}}(1+\log g(t))
$$

для всех $t>0$. 
ДокаЗАТЕЛЬСТво. Ввиду условия $\|v\|_{\mathbf{L}^{\infty}}+\|v\|_{\mathbf{L}^{1}} \leqslant C \varepsilon$ получаем

$$
\left.\left|\frac{2 \lambda}{\theta n} \int_{0}^{t} d \tau \int_{\mathbb{R}^{n}}\right| v\right|^{\frac{2}{n}} v(\tau, x) d x \mid \leqslant C \varepsilon^{\frac{2}{n}} t,
$$

откуда приходим к оценке (46) при всех $0<t<1$.

Рассмотрим теперь $t \geqslant 1$. В силу леммы 1 имеем

$$
\left\|\mathscr{G}(t) u_{0}-\theta G_{0}(t, x)\right\|_{\mathbf{L}^{1}} \leqslant C \varepsilon t^{-\frac{a}{2}},
$$

откуда находим

$$
\begin{aligned}
& \left\||v|^{\frac{2}{n}} v-|\theta|^{\frac{2}{n}} \theta\left(G_{0}(t, x)\right)^{1+\frac{2}{n}}\right\|_{\mathbf{L}^{1}} \\
& \leqslant C\left(\left\|v(t)-\mathscr{G}(t) u_{0}\right\|_{\mathbf{L}^{1}}+\left\|\mathscr{G}(t) u_{0}-\theta G_{0}(t, x)\right\|_{\mathbf{L}^{1}}\right) \\
& \quad \times\left(\|v\|_{\mathbf{L}^{\infty}}^{\frac{2}{n}}+\left\|\mathscr{G}(t) u_{0}\right\|_{\mathbf{L}^{\infty}}^{\frac{2}{n}}+|\theta|^{\frac{2}{n}}\left\|G_{0}(t)\right\|_{\mathbf{L}^{\infty}}^{\frac{2}{n}}\right) \\
& \leqslant C \varepsilon^{1+\frac{2}{n}} t^{-1}\left(\varepsilon^{\frac{2}{n}} g^{-1}(t)+t^{-\frac{a}{2}}\right)
\end{aligned}
$$

для всех $t \geqslant 1$. Поскольку

$$
t \int_{\mathbb{R}^{n}}\left(G_{0}(t, x)\right)^{1+\frac{2}{n}} d x=\frac{1}{4 \pi \alpha}\left(1+\frac{2}{n}\right)^{-\frac{n}{2}}=\frac{\eta}{2 \lambda},
$$

находим

$$
\begin{aligned}
& \left.\left|\int_{\mathbb{R}^{n}}\right| v\right|^{\frac{2}{n}} v(t, x) d x-|\theta|^{\frac{2}{n}} \theta t^{-1} \frac{\eta}{2 \lambda} \mid \\
& \quad \leqslant C\left\||v|^{\frac{2}{n}} v-|\theta|^{\frac{2}{n}} \theta\left(G_{0}(t, x)\right)^{1+\frac{2}{n}}\right\|_{\mathbf{L}^{1}} \\
& \quad \leqslant C \varepsilon^{1+\frac{2}{n}} t^{-1}\left(\varepsilon^{\frac{2}{n}} g^{-1}(t)+t^{-\frac{a}{2}}\right)
\end{aligned}
$$

для всех $t \geqslant 1$. Следовательно,

$$
\begin{aligned}
& \left.\left|\frac{2 \lambda}{\theta} \int_{1}^{t} d \tau \int_{\mathbb{R}^{n}}\right| v\right|^{\frac{2}{n}} v(\tau, x) d x-|\theta|^{\frac{2}{n}} \eta \log t \mid \\
& \quad \leqslant \int_{1}^{t} \frac{C \varepsilon^{\frac{4}{n}} d \tau}{\tau\left(1+\theta^{\frac{2}{n}} \eta \log (1+\tau)\right)}+C \varepsilon^{\frac{2}{n}} \int_{1}^{t} \tau^{-1-\frac{a}{2}} d \tau \\
& \quad \leqslant C \varepsilon^{\frac{2}{n}}(1+\log g(t))
\end{aligned}
$$

для всех $t \geqslant 1$. Таким образом, получаем (46). Лемма доказана.

В силу (45), применяя лемму 6 , находим

$$
\left|h_{m}(t)-g(t)\right| \leqslant C \varepsilon^{\frac{2}{n}}(1+\log g(t))
$$


для всех $t>0$. Таким образом, по индукции получаем оценки (42) для всех $m \geqslant 1$. Аналогично, по индукции доказьваются неравенства

$$
\begin{gathered}
\left\|v_{m}-v_{m-1}\right\| \mathbf{x} \leqslant \frac{1}{4}\left\|v_{m-1}-v_{m-2}\right\| \mathbf{x}, \\
\sup _{t>0} g^{-1}(t)\left|h_{m}(t)-h_{m-1}(t)\right| \leqslant \frac{1}{4}\left\|v_{m-1}-v_{m-2}\right\|_{\mathbf{X}} \\
+\frac{1}{4} \sup _{t>0} g^{-1}(t)\left|h_{m-1}(t)-h_{m-2}(t)\right|
\end{gathered}
$$

при всех $m>2$. Таким образом, переходя к пределу в $\mathbf{X}$ при $m \rightarrow \infty$, получаем единственное решение $\lim _{m \rightarrow \infty} v_{m}(t, x)=v(t, x) \in \mathbf{X}, \lim _{m \rightarrow \infty} h_{m}(t)=h(t)=$ $e^{\frac{2}{n} \varphi(t)} \in \mathbf{C}(0, \infty)$, удовлетворяюшее уравнениям

$$
\begin{gathered}
v(t)=\mathscr{G}(t) u_{0}+\int_{0}^{t} \mathscr{G}(t-\tau) \mathscr{B} f(v(\tau), h(\tau)) d \tau, \\
h(t)=1-\frac{2 \lambda}{\theta n} \int_{0}^{t} d \tau \int_{\mathbb{R}^{n}}|v|^{\frac{2}{n}} v d x
\end{gathered}
$$

и оценкам

$$
\begin{gathered}
\left\|v(t)-\mathscr{G}(t) u_{0}\right\|_{\mathbf{L}^{1}} \leqslant C \varepsilon^{1+\frac{2}{n}} g^{-1}(t), \\
\left|h_{m}(t)-g(t)\right| \leqslant C \varepsilon^{\frac{2}{n}}(1+\log g(t)) .
\end{gathered}
$$

Применяя (43) и (44) к уравнениям (47), находим

$$
\left\|v(t)-\mathscr{G}(t) u_{0}\right\|_{\mathbf{L}^{\infty}} \leqslant C \varepsilon^{1+\frac{2}{n}}\langle t\rangle^{-\frac{n}{2}} g^{-1}(t) .
$$

Отсюда, пользуясь формулами

$$
u(t, x)=e^{-\varphi(t)} v(t, x)=h^{-\frac{n}{2}}(t) v(t, x),
$$

получаем оценку

$$
\begin{aligned}
& \left\|u(t)-\theta G_{0}(t, x) e^{-\varphi(t)}\right\|_{\mathbf{L}^{\infty}} \\
& \quad \leqslant\left\|u(t)-\left(\mathscr{G}(t) u_{0}\right) e^{-\varphi(t)}\right\|_{\mathbf{L}^{\infty}}+\left\|\left(\mathscr{G}(t) u_{0}-\theta G_{0}(t, x)\right) e^{-\varphi(t)}\right\|_{\mathbf{L}^{\infty}} \\
& \quad \leqslant C \varepsilon^{1+\frac{2}{n}}\langle t\rangle^{-\frac{n}{2}} g^{-1-\frac{n}{2}}(t),
\end{aligned}
$$

где мы учли неравенство

$$
\left\|\left(\mathscr{G}(t) u_{0}-\theta G_{0}(t, x)\right) e^{-\varphi(t)}\right\|_{\mathbf{L}^{\infty}} \leqslant C t^{-\frac{n}{2}-\frac{a}{2}}\left\|u_{0}\right\|_{\mathbf{L}^{1, a}}
$$

и соотношение (49). Также ввиду (48) имеем

$$
\begin{gathered}
\left\|\theta G_{0}(t) h^{-\frac{n}{2}}(t)-\theta G_{0}(t, x) g^{-\frac{n}{2}}(t)\right\|_{\mathbf{L}^{\infty}} \\
\leqslant C \varepsilon t^{-\frac{n}{2}} g^{-1-\frac{n}{2}}(t)|h(t)-g(t)|,
\end{gathered}
$$

откуда в силу (50) вытекает

$$
\left\|u(t)-\theta G_{0}(t, x) g^{-\frac{n}{2}}(t)\right\|_{\mathbf{L}^{\infty}} \leqslant C \varepsilon^{1+\frac{2}{n}}\langle t\rangle^{-\frac{n}{2}} g^{-1-\frac{n}{2}}(t) \log g(t),
$$

что и завершает доказательство теоремы. 
4.2. Большие начальные данные. Здесь мы освободимся от условия малости начальных данных $u_{0}(x)$.

ТеОрема 4. Пусть $\lambda<0, n=1,2$. Предположим, что начальные данные $u_{0} \in \mathbf{W}_{\infty}^{2}\left(\mathbb{R}^{n}\right) \cap \mathbf{W}_{1}^{2, a}\left(\mathbb{R}^{n}\right), \quad 0<a \leqslant 1$, таковьи, что $\theta=\int_{\mathbb{R}^{n}} u_{0}(x) d x \neq 0$. Тогда задача Коши (1) при $\sigma=\frac{2}{n}$ имеет единственное глобальное во времени решение $u(t, x) \in \mathbf{C}\left([0, \infty) ; \mathbf{C}\left(\mathbb{R}^{n}\right) \cap \mathbf{L}^{\infty}\left(\mathbb{R}^{n}\right) \cap \mathbf{L}^{1, a}\left(\mathbb{R}^{n}\right)\right)$. Более того, имеет место асимптотика (38).

Прежде чем доказывать теорему 4, мы сравним решения следующих двух задач:

$$
\begin{gathered}
u_{t}-\alpha \Delta u+|u|^{\sigma} u=f(t, x), \quad x \in \mathbb{R}^{n}, \quad t>0, \\
u(0, x)=u_{0}(x), \quad x \in \mathbb{R}^{n},
\end{gathered}
$$

и

$$
\begin{gathered}
v_{t}-\alpha \Delta v+\varepsilon v^{\sigma+1}=|f(t, x)|, \quad x \in \mathbb{R}^{n}, \quad t>0, \\
v(0, x)=\mu\left|u_{0}(x)\right|, \quad x \in \mathbb{R}^{n},
\end{gathered}
$$

где $\alpha>0$. Отметим, что решение $v(t, x)$ задачи (53) остается положительным для всех $t \geqslant 0, x \in \mathbb{R}^{n}$ (см. [39]).

ЛЕмма 7. Пусть $0<\sigma \leqslant \frac{2}{n}$. Предположим, что $0 \leqslant \varepsilon \leqslant 1, \mu \geqslant 1$, начальнье данные $u_{0}(x) \in \mathbf{C}\left(\mathbb{R}^{n}\right) \cap \mathbf{L}^{\infty}\left(\mathbb{R}^{n}\right) \cap \mathbf{L}^{1, a}\left(\mathbb{R}^{n}\right)$ u $f(t, x) \in \mathbf{C}\left([0, \infty) ; \mathbf{C}\left(\mathbb{R}^{n}\right) \cap\right.$ $\left.\mathbf{L}^{\infty}\left(\mathbb{R}^{n}\right) \cap \mathbf{L}^{1, a}\left(\mathbb{R}^{n}\right)\right)$. Тогда для решений задач (52) и (53) справедливо неравенство $|u(t, x)| \leqslant v(t, x)$ для всех $t \geqslant 0, \quad x \in \mathbb{R}^{n}$.

ДоКАЗАТЕЛЬСТВо. Обозначив $r=v-u$, из (52) и (53) получим

$$
\begin{gathered}
r_{t}-\alpha \Delta r+\varepsilon v^{\sigma+1}-|u|^{\sigma} u=|f|-f, \quad x \in \mathbb{R}^{n}, \quad t>0, \\
r(0, x)=\mu\left|u_{0}(x)\right|-u_{0}(x), \quad x \in \mathbb{R}^{n} .
\end{gathered}
$$

Рассмотрим $R(t) \equiv \inf _{x \in \mathbb{R}^{n}} r(t, x)$. Отметим, что из-за сглаживающего эффекта, характерного для уравнения теплопроводности (см. [39]), функция $R(t)$ является непрерывной по времени. Нам нужно доказать, что $R(t) \geqslant 0$ для всех $t \geqslant 0$. Предположим от противного, что $R(T)<0$ для некоторого $T>0$. По непрерьвности мы можем найти отрезок времени $\left[T_{1}, T\right]$ такой, что $R(t) \leqslant 0$ для всех $t \in\left[T_{1}, T\right]$ и $R\left(T_{1}\right)=0$. По теореме 2.1 из работы [6] сушествует кривая $\zeta(t) \in \mathbb{R}^{n}$ такая, что $R(t)=r(t, \zeta(t))$, причем $R^{\prime}(t)=\frac{d}{d t} r(t, \zeta(t))=r_{t}(t, \zeta(t))$ почти на всем сегменте $\left[T_{1}, T\right]$. Имеем на кривой $x=\zeta(t)$

$$
|u|^{\sigma} u-\varepsilon v^{\sigma+1}=(v-R)^{\sigma+1}-\varepsilon v^{\sigma+1} \geqslant 0
$$

для всех $t \in\left[T_{1}, T\right]$. Для лапласиана $\Delta r$ в точках минимума $\zeta(t)$ имеем

$$
-\left.\Delta r(t, x)\right|_{x=\zeta(t)} \leqslant 0 .
$$

Поэтому из уравнения (54) получаем

$$
R^{\prime}(t) \geqslant 0
$$

почти для всех $t \in\left[T_{1}, T\right]$, откуда интегрирование по времени дает $R(t) \geqslant 0$. Это противоречит предположению, значит, $u(t, x) \leqslant v(t, x)$ для всех $x \in \mathbb{R}^{n}$ и $t \geqslant 0$. Аналогично доказьвается, что $v+u \geqslant 0$ для всех $x \in \mathbb{R}^{n}$ и $t \geqslant 0$. Лемма доказана. 
ДОКАЗАТЕЛЬСТВО ТЕОРЕМЫ 4 . Как и при доказательстве теоремы 2 , пользуясь леммой 1 и утверждением 2, при $\sigma=\frac{2}{n}$ найдем

$$
\begin{aligned}
\|u(t)\|_{\mathbf{L}^{\infty}} \leqslant & \left\|\mathscr{G}(t) u_{0}\right\|_{\mathbf{L}^{\infty}}+C \int_{\frac{t}{2}}^{t}\langle t-\tau\rangle^{-\frac{n(\sigma+1)}{2(\sigma+2)}}\|u(\tau)\|_{\mathbf{L}^{\sigma+2}}^{\sigma+1} d \tau \\
& +C \int_{0}^{\frac{t}{2}}\langle t-\tau\rangle^{-\frac{n}{2}}\left(\left\||u|^{\sigma} u(\tau)\right\|_{\mathbf{L}^{1}}+\|u(\tau)\|_{\mathbf{L}^{\sigma+2}}^{\sigma+1}\right) d \tau \\
\leqslant & C\langle t\rangle^{-\frac{n}{2}}+C \int_{\frac{t}{2}}^{t}\langle t-\tau\rangle^{-\frac{n(\sigma+1)}{2(\sigma+2)}}\langle\tau\rangle^{-\frac{n(\sigma+1)^{2}}{2(\sigma+2)}} d \tau \\
& +C \int_{0}^{\frac{t}{2}}\langle t-\tau\rangle^{-\frac{n}{2}}\langle\tau\rangle^{-1} d \tau \leqslant C\langle t\rangle^{-\frac{n}{2}} \log \langle t\rangle
\end{aligned}
$$

для всех $t>0$. Из интегрального уравнения (10) следует, что решение $u(t)$ является гладким: $u \in \mathbf{C}\left([0, \infty) ; \mathbf{W}_{\infty}^{2}\left(\mathbb{R}^{n}\right) \cap \mathbf{W}_{1}^{2}\left(\mathbb{R}^{n}\right)\right)$, поскольку начальные данные $u_{0} \in \mathbf{W}_{\infty}^{2}\left(\mathbb{R}^{n}\right) \cap \mathbf{W}_{1}^{2, a}\left(\mathbb{R}^{n}\right)$. Поэтому, взяв лапласиан от уравнения (10), ввиду (55) получим

$$
\begin{aligned}
\|\Delta u(t)\|_{\mathbf{L}} \leqslant & C\langle t\rangle^{-1-\frac{n}{2}}+\int_{0}^{t}\left\|\Delta \mathscr{G}(t-\tau) \mathscr{B}|u|^{\sigma} u(\tau)\right\|_{\mathbf{L} \infty d \tau} \\
\leqslant & C\langle t\rangle^{-1-\frac{n}{2}}+C \int_{0}^{\frac{t}{2}}\langle t-\tau\rangle^{-1-\frac{n}{2}}\|u(\tau)\|_{\mathbf{L}^{\sigma+1}}^{\sigma+1} d \tau \\
& +C \int_{\frac{t}{2}}^{t}\langle t-\tau\rangle^{-1}\|u(\tau)\|_{\mathbf{L}^{\infty}}^{\sigma+1} d \tau \\
\leqslant & C\langle t\rangle^{-1-\frac{n}{2}}+C \int_{0}^{\frac{t}{2}}\langle t-\tau\rangle^{-1-\frac{n}{2}}\langle\tau\rangle^{-1} d \tau \\
& +C \int_{\frac{t}{2}}^{t}\langle t-\tau\rangle^{-1}\langle\tau\rangle^{-1-\frac{n}{2}} \log ^{\sigma+1}\langle\tau\rangle d \tau \leqslant C\langle t\rangle^{-1-\frac{n}{2}} \log ^{\sigma+2}\langle t\rangle
\end{aligned}
$$

для всех $t>0$. Обозначим $f(t, x)=\Delta u_{t}$. Поскольку $\partial_{t} \mathscr{G}(t)=\Delta \mathscr{B} \mathscr{G}(t)$, то из интегрального уравнения (10) получаем

$$
\begin{aligned}
\Delta u_{t}(t)= & \Delta^{2} \mathscr{B} \mathscr{G}(t) u_{0}+\lambda \Delta \mathscr{B}|u(t)|^{\frac{2}{n}} u(t) \\
& +\lambda \int_{\frac{t}{2}}^{t} \Delta \mathscr{B} \mathscr{G}(t-\tau) \Delta \mathscr{B}|u(\tau)|^{\frac{2}{n}} u(\tau) d \tau \\
& +\lambda \int_{0}^{\frac{t}{2}}(\Delta \mathscr{B})^{2} \mathscr{G}(t-\tau)|u(\tau)|^{\frac{2}{n}} u(\tau) d \tau .
\end{aligned}
$$

Поскольку оператор $\Delta \mathscr{B}=\mathscr{B}-1$ ограничен, а начальные данные $u_{0} \in \mathbf{W}_{\infty}^{2}\left(\mathbb{R}^{n}\right) \cap$ $\mathbf{W}_{1}^{2, a}\left(\mathbb{R}^{n}\right)$, заключаем, что $\Delta u_{t}(t) \in \mathbf{L}^{\infty}\left(\mathbb{R}^{n}\right) \cap \mathbf{L}^{1, a}\left(\mathbb{R}^{n}\right)$. Применяя теперь оценки 
леммы 1, находим

$$
\begin{aligned}
\|f(t)\|_{\mathbf{L}^{\infty}}= & \left\|\Delta u_{t}(t)\right\|_{\mathbf{L}^{\infty}} \leqslant\left\|\Delta^{2} \mathscr{B} \mathscr{G}(t) u_{0}\right\|_{\mathbf{L}^{\infty}}+\left\|\lambda \Delta \mathscr{B}|u(t)|^{\frac{2}{n}} u(t)\right\|_{\mathbf{L}^{\infty}} \\
& +C \int_{\frac{t}{2}}^{t}\langle t-\tau\rangle^{-1}\left\|\Delta \mathscr{B}|u|^{\frac{2}{n}} u(\tau)\right\|_{\mathbf{L}^{\infty}} d \tau \\
& +C \int_{0}^{\frac{t}{2}}\langle t-\tau\rangle^{-\frac{n}{2}-2}\left(\left\||u|^{\frac{2}{n}} u(\tau)\right\|_{\mathbf{L}^{1}}+\left\|\mathscr{B}|u|^{\frac{2}{n}} u(\tau)\right\|_{\mathbf{L}^{\infty}}\right) d \tau \\
\leqslant & C\langle t\rangle^{-2-\frac{n}{2}} \log ^{2 \sigma+2}\langle t\rangle+C \int_{\frac{t}{2}}^{t}\langle t-\tau\rangle^{-1}\langle\tau\rangle^{-2-\frac{n}{2}} \log ^{2 \sigma+2}\langle\tau\rangle d \tau \\
& +C \int_{0}^{\frac{t}{2}}\langle t-\tau\rangle^{-\frac{n}{2}-2}\langle\tau\rangle^{-1} \log \langle\tau\rangle d \tau \leqslant C\langle t\rangle^{-2-\frac{n}{2}} \log ^{2 \sigma+2}\langle t\rangle
\end{aligned}
$$

для всех $t>0$. Возьмем достаточно малое $\varepsilon>0$ и рассмотрим следующие две вспомогательные задачи Коши

$$
\begin{gathered}
U_{t}-\alpha \Delta U+U^{\sigma+1}=\varepsilon^{2}|f(t, x)|, \quad x \in \mathbb{R}^{n}, \quad t>0, \\
U(0, x)=\varepsilon\left|u_{0}(x)\right|, \quad x \in \mathbb{R}^{n},
\end{gathered}
$$

И

$$
\begin{gathered}
V_{t}-\alpha \Delta V+\varepsilon^{2 \sigma} V^{\sigma+1}=|f(t, x)|, \quad x \in \mathbb{R}^{n}, \quad t>0, \\
V(0, x)=\frac{1}{\varepsilon}\left|u_{0}(x)\right|, \quad x \in \mathbb{R}^{n} .
\end{gathered}
$$

Заметим, что задача (57) может быть сведена к задаче (56) с помощью замены $V=\varepsilon^{-2} U$. В свою очередь, задача (56) имеет достаточно малые начальные данные и малую правую часть $\varepsilon^{2}|f|$, причем общая масса начальных данных

$$
\int_{\mathbb{R}^{n}} U(0, x) d x=\int_{\mathbb{R}^{n}} \varepsilon\left|u_{0}(x)\right| d x
$$

имеет порядок $O(\varepsilon)$, так что среднее значение $\frac{1}{\theta} \int_{\mathbb{R}^{n}} \varepsilon^{2}|f(t, x)| d x$ также является малым. Применение принципа максимума к задаче (56) (см., например, [39]) дает почти точную оценку убывания по времени (в точной оценке добавляется логарифмическое убывание)

$$
\|U(t)\|_{\mathbf{L}} \leqslant C\langle t\rangle^{-\frac{n}{2}}
$$

откуда с помощью леммы 7 находим

$$
\|u(t)\|_{\mathbf{L}^{\infty}} \leqslant C\langle t\rangle^{-\frac{n}{2}} .
$$

Теперь оценим $\mathbf{L}^{1, a}$-норму решения. В силу (5) и (6), принимая во внимание (58) и результат утверждения 2 , имеем

$$
\begin{aligned}
\left\|\mathscr{B}|u|^{\sigma} u(\tau)\right\|_{\mathbf{L}^{1}} & \leqslant C\|u(\tau)\|_{\mathbf{L}^{\infty}}^{\sigma}\|u(\tau)\|_{\mathbf{L}^{1}} \\
& \leqslant C\langle\tau\rangle^{-\frac{n}{2} \sigma}\|u(\tau)\|_{\mathbf{L}^{1}} \leqslant C\langle\tau\rangle^{-1}, \\
\left\|\mathscr{B}|u|^{\sigma} u(\tau)\right\|_{\mathbf{L}^{1, a}} & \leqslant C\|u(\tau)\|_{\mathbf{L}^{\infty}}^{\sigma}\|u(\tau)\|_{\mathbf{L}^{1, a}} \\
& \leqslant C\langle\tau\rangle^{-\frac{n}{2} \sigma}\|u(\tau)\|_{\mathbf{L}^{1, a}} \leqslant C\langle\tau\rangle^{-1}\|u(\tau)\|_{\mathbf{L}^{1, a}},
\end{aligned}
$$


поэтому

$$
\begin{aligned}
\|u(t)\|_{\mathbf{L}^{1, a}} \leqslant & \left\|\mathscr{G}(t) u_{0}\right\|_{\mathbf{L}^{1, a}} \\
& +C \int_{0}^{t}\left(\left\|\mathscr{B}|u|^{\sigma} u(\tau)\right\|_{\mathbf{L}^{1, a}}+\langle t-\tau\rangle^{\frac{a}{2}}\left\|\mathscr{B}|u|^{\sigma} u(\tau)\right\|_{\mathbf{L}^{1}}\right) d \tau \\
\leqslant & C\langle t\rangle^{\frac{a}{2}}+C \int_{0}^{t}\langle\tau\rangle^{-1}\|u(\tau)\|_{\mathbf{L}^{1, a}} d \tau+C \int_{0}^{t}\langle t-\tau\rangle^{\frac{a}{2}}\langle\tau\rangle^{-1} d \tau \\
\leqslant & C\langle t\rangle^{\frac{a}{2}}+C \int_{0}^{t}\langle\tau\rangle^{-1}\|u(\tau)\|_{\mathbf{L}^{1, a}} d \tau .
\end{aligned}
$$

Откуда с помощью леммы Гронуолла получаем

$$
\|u(t)\|_{\mathbf{L}^{1, a}} \leqslant C\langle t\rangle^{\frac{a}{2}}
$$

для всех $t>0$. Аналогично оценим $\mathbf{L}^{1, a}$-норму функции $f$

$$
\begin{aligned}
\|f(t)\|_{\mathbf{L}^{1, a}}= & \left\|\Delta u_{t}(t)\right\|_{\mathbf{L}^{1, a}} \\
\leqslant & \left\|\Delta^{2} \mathscr{B} \mathscr{G}(t) u_{0}\right\|_{\mathbf{L}^{1, a}}+C\left\|\Delta \mathscr{B}|u|^{\frac{2}{n}} u(t)\right\|_{\mathbf{L}^{1, a}} \\
& \quad+C \int_{0}^{t}\langle t-\tau\rangle^{-1}\left(\left\||u|^{\frac{2}{n}} u(\tau)\right\|_{\mathbf{L}^{1, a}}+\langle t-\tau\rangle^{\frac{a}{2}}\left\||u|^{\frac{2}{n}} u(\tau)\right\|_{\mathbf{L}^{1}}\right) d \tau \\
\leqslant & C\langle t\rangle^{-1+\frac{a}{2}}+C \int_{0}^{t}\langle t-\tau\rangle^{-1}\langle\tau\rangle^{\frac{a}{2}-1} d \tau+C \int_{0}^{t}\langle t-\tau\rangle^{\frac{a}{2}-1}\langle\tau\rangle^{-1} d \tau \\
\leqslant & C\langle t\rangle^{-1+\frac{a}{2}} .
\end{aligned}
$$

Таким образом, мы можем использовать результаты работ [21], [22], чтобы вычислить асимптотическое поведение при больших временах функций $U(t, x)$ и $V(t, x)$, откуда с помошью леммы 7 получаем точную оценку убывания по времени решения

$$
\|u(t)\|_{\mathbf{L}^{\infty}} \leqslant C\langle t\rangle^{-\frac{n}{2}}(\log (2+t))^{-\frac{n}{2}}
$$

для всех $t>0$.

Теперь, как и в доказательстве теоремы 3 , сделаем замену $u(t, x)=v(t, x) e^{-\varphi(t)}$. Тогда для новых функций $(v(t, x), \varphi(t))$ получаем задачу (39). Докажем оценку

$$
\|v(t)\|_{\mathbf{L}^{1, a}} \leqslant C\langle t\rangle^{\frac{a}{2}}
$$

для всех $t>0$. Воспользуемся интегральным уравнением, вытекающим из (39):

$$
\begin{gathered}
v(t)=\mathscr{G}(t) u_{0}+\lambda \int_{0}^{t} \mathscr{G}(t-\tau) \mathscr{B}\left(|u(\tau)|^{\sigma} v(\tau)\right. \\
\left.-\frac{v(\tau)}{\theta} \int_{\mathbb{R}^{n}}|u(\tau)|^{\sigma} v(\tau) d x\right) d \tau .
\end{gathered}
$$


Из неравенства (60) находим

$$
\|u(t)\|_{\mathbf{L}^{\infty}}^{\sigma} \leqslant C\langle t\rangle^{-1}(\log (2+t))^{-1},
$$

поэтому в силу леммы 1 получаем

$$
\begin{aligned}
& \left\|\int_{0}^{t} \mathscr{G}(t-\tau) \mathscr{B}\left(|u(\tau)|^{\sigma} v(\tau)-\frac{v(\tau)}{\theta} \int_{\mathbb{R}^{n}}|u(\tau)|^{\sigma} v(\tau) d x\right) d \tau\right\|_{\mathbf{L}^{1, a}} \\
& \quad \leqslant C \int_{0}^{t}\left\||u(\tau)|^{\sigma} v(\tau)-\frac{v(\tau)}{\theta} \int_{\mathbb{R}^{n}}|u(\tau)|^{\sigma} v(\tau) d x\right\|_{\mathbf{L}^{1, a}} d \tau \\
& \quad \leqslant C \int_{0}^{t}\langle\tau\rangle^{-1}(\log (2+\tau))^{-1}\|v(\tau)\|_{\mathbf{L}^{1, a}} d \tau
\end{aligned}
$$

для всех $t>0$. Следовательно, ввиду (59) находим

$$
\begin{aligned}
\|v(t)\|_{\mathbf{L}^{1, a}} \leqslant & \|\mathscr{G}(t) v(0)\|_{\mathbf{L}^{1, a}} \\
& +C \int_{0}^{t}\langle\tau\rangle^{-1}(\log (2+\tau))^{-1}\|v(\tau)\|_{\mathbf{L}^{1, a}} d \tau \\
\leqslant & C\langle t\rangle^{\frac{a}{2}}+C \int_{0}^{t}\langle\tau\rangle^{-1}(\log (2+\tau))^{-1}\|v(\tau)\|_{\mathbf{L}^{1, a}} d \tau
\end{aligned}
$$

для всех $t>0$, откуда, применяя лемму Гронуолла, приходим к оценке

$$
\|v(t)\|_{\mathbf{L}^{1, a}} \leqslant C\langle t\rangle^{\frac{a}{2}}
$$

для всех $t>0$. Аналогично, с помощью оценки (60) и леммы 1 получим

$$
\begin{aligned}
\|v(t)\|_{\mathbf{L}^{p} \leqslant} \leqslant & \|\mathscr{G}(t) v(0)\|_{\mathbf{L}^{p}} \\
& +\left\|\int_{0}^{t} \mathscr{G}(t-\tau) \mathscr{B}\left(|u(\tau)|^{\sigma} v(\tau)-\frac{v(\tau)}{\theta} \int_{\mathbb{R}^{n}}|u(\tau)|^{\sigma} v(\tau) d x\right) d \tau\right\|_{\mathbf{L}^{p}} \\
\leqslant & C\langle t\rangle^{-\frac{n}{2}\left(1-\frac{1}{p}\right)} \\
& +C \int_{0}^{\frac{t}{2}}(t-\tau)^{-\frac{n}{2}\left(1-\frac{1}{p}\right)-\frac{a}{2}}\langle\tau\rangle^{\frac{a}{2}-1}(\log (2+\tau))^{-1} d \tau \\
& +C \int_{\frac{t}{2}}^{t}\langle\tau\rangle^{-1}(\log (2+\tau))^{-1}\|v(\tau)\|_{\mathbf{L}^{p}} d \tau \\
\leqslant & C\langle t\rangle^{-\frac{n}{2}\left(1-\frac{1}{p}\right)}+\int_{\frac{t}{2}}^{t}\langle\tau\rangle^{-1}\|v(\tau)\|_{\mathbf{L}^{p}} d \tau
\end{aligned}
$$

для всех $t>0$. Откуда лемма Гронуолла приводит к оценке

$$
\|v(t)\|_{\mathbf{L}^{p}} \leqslant C\langle t\rangle^{-\frac{n}{2}\left(1-\frac{1}{p}\right)}
$$

для всех $t>0$. 
Из интегрального уравнения (61) и леммы 1 получаем теперь

$$
\begin{aligned}
& \left\|v(t)-\mathscr{G}(t) u_{0}\right\|_{\mathbf{L}^{1}} \leqslant \int_{0}^{\frac{t}{2}}(t-\tau)^{-\frac{a}{2}}\left\||u(\tau)|^{\sigma} v(\tau)-\frac{v(\tau)}{\theta} \int_{\mathbb{R}^{n}}|u(\tau)|^{\sigma} v(\tau) d x\right\|_{\mathbf{L}^{1, a}} d \tau \\
& +\int_{\frac{t}{2}}^{t}\left\||u(\tau)|^{\sigma} v(\tau)-\frac{v(\tau)}{\theta} \int_{\mathbb{R}^{n}}|u(\tau)|^{\sigma} v(\tau) d x\right\|_{\mathbf{L}^{1}} d \tau \\
& \leqslant C \int_{0}^{\frac{t}{2}}(t-\tau)^{-\frac{a}{2}}\langle\tau\rangle^{\frac{a}{2}-1}(\log (2+\tau))^{-1} d \tau \\
& +C \int_{\frac{t}{2}}^{t}\langle\tau\rangle^{-1}(\log (2+\tau))^{-1} d \tau \leqslant C \log (2+t)^{-1}
\end{aligned}
$$

для всех $t>0$. Поэтому, применив лемму 6 к функции $h(t)=e^{\sigma \varphi(t)}$, найдем

$$
|h(t)-g(t)| \leqslant C \log g(t)
$$

для всех $t>0$. Тогда из формул $u(t, x)=e^{-\varphi(t)} v(t, x)=h^{-\frac{n}{2}}(t) v(t, x)$ находим оценку

$$
\begin{aligned}
\| u(t) & -\theta e^{-\varphi(t)} G_{0}(t)\left\|_{\mathbf{L}^{\infty}} \leqslant\right\| u(t)-e^{-\varphi(t)} \mathscr{G}_{(t)} u_{0} \|_{\mathbf{L}^{\infty}} \\
& +\left\|\mathscr{G}(t) u_{0}-\theta e^{-\varphi(t)} G_{0}(t)\right\|_{\mathbf{L}^{\infty}} \\
\leqslant & C t^{-\frac{n}{2}} g^{-1-\frac{n}{2}}(t)+C t^{-\frac{n}{2}-\frac{a}{2}}\left\|u_{0}\right\|_{\mathbf{L}^{1, a}} \leqslant C t^{-\frac{n}{2}} g^{-1-\frac{n}{2}}(t)
\end{aligned}
$$

для всех $t>0$. Также имеем

$$
\left\|\theta G_{0}(t)\left(h^{-\frac{n}{2}}(t)-g^{-\frac{n}{2}}(t)\right)\right\|_{\mathbf{L}^{\infty}} \leqslant C t^{-\frac{n}{2}} g^{-1-\frac{n}{2}}(t)|h(t)-g(t)|,
$$

откуда ввиду (63) получаем

$$
\left\|u(t)-\theta G_{0}(t) g^{-\frac{n}{2}}(t)\right\|_{\mathbf{L}^{\infty}} \leqslant C(1+t)^{-\frac{n}{2}} g^{-1-\frac{n}{2}}(t) \log g(t),
$$

что завершает доказательство теоремы.

\section{§5. Субкритический случай}

В настояшем параграфе мы изучим задачу Коши (1) в субкритическом случае $\sigma<\frac{2}{n}$.

5.1. Малые начальные данные. Сначала мы докажем существование глобальных во времени малых решений задачи Коши (1) в субкритическом случае $0<\sigma<\frac{2}{n}$. Обозначим

$$
\widetilde{G}(x)=(4 \pi \alpha)^{-\frac{n}{2}} e^{-\frac{|x|^{2}}{4 \alpha}}=G_{0}(1, x) .
$$


Tеорема 5. Пусть $\lambda<0, \quad 0<\sigma<\frac{2}{n}$. Предположим, что начальные данные $u_{0} \in \mathbf{C}\left(\mathbb{R}^{n}\right) \cap \mathbf{L}^{\infty}\left(\mathbb{R}^{n}\right) \cap \mathbf{L}^{1, a}\left(\mathbb{R}^{n}\right), \quad 0<a \leqslant 1$, достаточно маль: $\left\|u_{0}\right\|_{\mathbf{L}^{\infty}}+\left\|u_{0}\right\|_{\mathbf{L}^{1, a}} \leqslant \varepsilon$, и таковй, что $\theta \geqslant C \varepsilon>0$, где $\theta=\int_{\mathbb{R}^{n}} u_{0}(x) d x$. Также предположим, что значение $\sigma$ близко $\kappa \frac{2}{n}$, так что $\frac{2}{n}-\sigma \leqslant C \varepsilon^{\sigma}$. Тогда задача Коши (1) имеет единственное глобальное во времени решение $u(t, x) \in \mathbf{C}\left([0, \infty) ; \mathbf{C}\left(\mathbb{R}^{n}\right) \cap \mathbf{L}^{\infty}\left(\mathbb{R}^{n}\right) \cap \mathbf{L}^{1, a}\left(\mathbb{R}^{n}\right)\right)$ и справедлива следующая оченка убивания решения $u(t, x)$ :

$$
\|u(t)\|_{\mathbf{L}^{\infty}} \leqslant C\langle t\rangle^{-\frac{1}{\sigma}}
$$

для всех $t>0$. Более того, существуют число $A$ и функиия $V \in \mathbf{C}\left(\mathbb{R}^{n}\right) \cap$ $\mathbf{L}^{\infty}\left(\mathbb{R}^{n}\right) \cap \mathbf{L}^{1, a}\left(\mathbb{R}^{n}\right)$ такие, что справедлива асимптотическая формула

$$
u(t, x)=A t^{-\frac{1}{\sigma}} V\left(x t^{-\frac{1}{2}}\right)+O\left(t^{-\frac{1}{\sigma}-\gamma}\right)
$$

при $t \rightarrow \infty$ равномерно по $x \in \mathbb{R}^{n}$, где $\gamma=\frac{1}{2} \min \left(a, 1-\frac{n \sigma}{2}\right), u V(\xi)$ является решением интегрального уравнения

$$
V(\xi)=\widetilde{G}(\xi)-\frac{1}{\beta} \int_{0}^{1} \frac{d z}{z(1-z)^{\frac{n}{2}}} \int_{\mathbb{R}^{n}} \widetilde{G}\left(\left(\xi-y z^{\frac{1}{2}}\right)(1-z)^{-\frac{1}{2}}\right) F(y) d y
$$

әде

$$
\begin{gathered}
\beta=\frac{\sigma}{1-\frac{n}{2} \sigma} \int_{\mathbb{R}^{n}} V^{1+\sigma}(y) d y \\
F(y)=V^{1+\sigma}(y)-V(y) \int_{\mathbb{R}^{n}} V^{1+\sigma}(\xi) d \xi .
\end{gathered}
$$

ЗАмЕчАниЕ 2. Условие, что значение $\sigma$ должно быть близким к величине $\frac{2}{n}$, так что $\frac{2}{n}-\sigma \leqslant C \varepsilon^{\sigma}$, является в известной мере техническим. Оно возникает из-за применения принципа сжимающих отображений при доказательстве существования глобального во времени решения.

ДОКАЗАТЕЛЬСТВО ТЕОРЕМЫ 5 . Как и при доказательстве теоремы 3 , сделаем замену $u(t, x)=v(t, x) e^{-\varphi(t)}$ и для новых функций $(v(t, x), \varphi(t))$ снова приходим к задаче Коши

$$
\begin{gathered}
\partial_{t}(v-\Delta v)-\alpha \Delta v=\lambda e^{-\sigma \varphi}\left(|v|^{\sigma}-\frac{1}{\theta} \int_{\mathbb{R}^{n}}|v|^{\sigma} v d x\right) v \\
\varphi^{\prime}(t)=-\frac{\lambda}{\theta} e^{-\sigma \varphi} \int_{\mathbb{R}^{n}}|v|^{\sigma} v d x \\
v(0, x)=u_{0}(x), \quad \varphi(0)=0
\end{gathered}
$$

Обозначим $h(t)=e^{\sigma \varphi(t)}$ и перепишем $(67)$ в виде

$$
\begin{gathered}
\partial_{t}(v-\Delta v)-\alpha \Delta v=f(v, h), \quad v(0, x)=u_{0}(x), \\
h^{\prime}=-\frac{\sigma \lambda}{\theta} \int_{\mathbb{R}^{n}}|v|^{\sigma} v d x, \quad h(0)=1,
\end{gathered}
$$


где

$$
f(v, h)=\lambda h^{-1}\left(|v|^{\sigma}-\frac{1}{\theta} \int_{\mathbb{R}^{n}}|v|^{\sigma} v d x\right) v .
$$

Заметим, что среднее значение нелинейности

$$
\int_{\mathbb{R}^{n}} f(v, h)(t, x) d x=0
$$

для всех $t>0$. Будем доказьвать сушествование решения $(v(t, x), h(t))$ задачи Коши (68) с помощью метода последовательных приближений. Обозначим $v_{1}=$ $\mathscr{G}(t) u_{0}$ и

$$
h_{1}(t)=1+\frac{2|\theta|^{\sigma} \eta}{2-n \sigma} t^{1-\frac{\sigma}{2} n}
$$

где

$$
\eta=\sigma \lambda(4 \pi \alpha)^{-\frac{n}{2} \sigma}(1+\sigma)^{-\frac{n}{2}} .
$$

Определим функции $\left(v_{m}(t, x), h_{m}(t)\right)$ для всех $m \geqslant 2$ как решение следуюшей линейной задачи (понимаемое в смысле соответствующего интегрального уравнения):

$$
\begin{gathered}
\partial_{t}\left(v_{m}-\Delta v_{m}\right)-\alpha \Delta v_{m}=f\left(v_{m-1}, h_{m-1}\right), \\
h_{m}^{\prime}=-\frac{\sigma \lambda}{\theta} \int_{\mathbb{R}^{n}}\left|v_{m-1}\right|^{\sigma} v_{m-1} d x, \\
v_{m}(0, x)=u_{0}(x), \quad h_{m}(0)=1 .
\end{gathered}
$$

Докажем по индукции следующие оценки:

$$
\begin{gathered}
\left\|v_{m}\right\| \mathbf{x} \leqslant C \varepsilon, \quad\left\|v_{m}(t)-\mathscr{G}(t) u_{0}\right\|_{\mathbf{L}^{1}} \leqslant C \varepsilon^{1+\sigma}, \\
\left|h_{m}(t)-h_{1}(t)\right| \leqslant C \varepsilon^{\sigma} h_{1}(t)
\end{gathered}
$$

для всех $m \geqslant 1$, где норма $\|\cdot\| \mathbf{x}$ задается, как и раньше, с помошью равенства

$$
\|\varphi\|_{\mathbf{X}}=\sup _{t>0}\left(\langle t\rangle^{\frac{n}{2}}\|\varphi(t)\|_{\mathbf{L}^{\infty}}+\langle t\rangle^{-\frac{a}{2}}\|\varphi(t)\|_{\mathbf{L}^{1, a}}\right) .
$$

Из леммы 1 имеем

$$
\begin{gathered}
\left\|\mathscr{G}(t) u_{0}\right\|_{\mathbf{L}^{\infty}} \leqslant C \varepsilon\langle t\rangle^{-\frac{n}{2}}, \quad\left\|\mathscr{G}(t) u_{0}\right\|_{\mathbf{L}^{1}} \leqslant C \varepsilon, \\
\left\||\cdot|^{a}\left(\mathscr{G}(t) u_{0}-\theta t^{-\frac{n}{2}} \widetilde{G}\left(t^{-\frac{1}{2}}(\cdot)\right)\right)\right\|_{\mathbf{L}^{1}} \leqslant C \varepsilon \\
\left\|t^{-\frac{n}{2}}|\cdot|^{a} \widetilde{G}\left(t^{-\frac{1}{2}}(\cdot)\right)\right\|_{\mathbf{L}^{1}} \leqslant C t^{\frac{a}{2}}
\end{gathered}
$$

откуда видим, что оценки (70) выполнены при $m=1$. Предположим по индукции, что оценки (70) верны для некоторого номера $m-1$. Запишем интегральное уравнение для задачи (69) в виде

$$
\begin{gathered}
v_{m}(t)=\mathscr{G}(t) u_{0}+\int_{0}^{t} \mathscr{G}(t-\tau) \mathscr{B} f\left(v_{m-1}(\tau), h_{m-1}(\tau)\right) d \tau \\
h_{m}(t)=1-\frac{\sigma \lambda}{\theta} \int_{0}^{t} d \tau \int_{\mathbb{R}^{n}}\left|v_{m-1}\right|^{\sigma} v_{m-1} d x
\end{gathered}
$$


Поскольку $\left(v_{m-1}(t), h_{m-1}(t)\right)$ удовлетворяют $(70)$, получаем

$$
\begin{aligned}
& \left\|f\left(v_{m-1}(t), h_{m-1}(t)\right)\right\|_{\mathbf{L}^{\infty}} \\
& \quad \leqslant C h_{m-1}^{-1}(t)\left\|v_{m-1}(t)\right\|_{\mathbf{L}^{\infty}}^{1+\sigma}\left(1+\frac{1}{|\theta|}\left\|v_{m-1}(t)\right\|_{\mathbf{L}^{1}}\right) \\
& \quad \leqslant C \varepsilon^{1+\sigma}\langle t\rangle^{-\frac{1+\sigma}{2} n}\left(1+\frac{n \sigma|\theta|^{\sigma} \eta}{2-n \sigma} t^{1-\frac{\sigma}{2} n}\right)^{-1} \\
& \quad \leqslant C \varepsilon\left(\frac{2}{n}-\sigma\right)\langle t\rangle^{-1-\frac{n}{2}}
\end{aligned}
$$

и, аналогично,

$$
\begin{aligned}
& \left\|f\left(v_{m-1}(t), h_{m-1}(t)\right)\right\|_{\mathbf{L}^{1, a}} \\
& \quad \leqslant C h_{m-1}^{-1}(t)\left\|v_{m-1}(t)\right\|_{\mathbf{L}^{\infty}}^{\sigma}\left\|v_{m-1}(t)\right\|_{\mathbf{L}^{1, a}}\left(1+\frac{1}{|\theta|}\left\|v_{m-1}(t)\right\|_{\mathbf{L}^{1}}\right) \\
& \quad \leqslant C \varepsilon\left(\frac{2}{n}-\sigma\right)\langle t\rangle^{-1+\frac{a}{2}}
\end{aligned}
$$

для всех $t>0$. Отсюда получаем оценку

$$
\left\|\langle t\rangle f\left(v_{m-1}(t), h_{m-1}(t)\right)\right\|_{\mathbf{X}} \leqslant C \varepsilon^{1+\sigma}
$$

так как мы предполагаем, что $2-n \sigma \leqslant C \varepsilon^{\sigma}$. Поскольку $f\left(v_{m-1}, h_{m-1}\right)$ имеет нулевое среднее значение:

$$
\begin{array}{rl}
\int_{\mathbb{R}^{n}} & f\left(v_{m-1}(t, x), h_{m-1}(t)\right) d x \\
= & \frac{\lambda}{h_{m-1}} \int_{\mathbb{R}^{n}}\left(\left|v_{m-1}\right|^{\sigma} v_{m-1}-\frac{v_{m-1}}{\theta} \int_{\mathbb{R}^{n}}\left|v_{m-1}\right|^{\sigma} v_{m-1} d x\right) d x \\
= & \frac{\lambda}{h_{m-1}} \int_{\mathbb{R}^{n}}\left|v_{m-1}\right|^{\sigma} v_{m-1} d x\left(1-\frac{1}{\theta} \int_{\mathbb{R}^{n}} v_{m-1} d x\right)=0
\end{array}
$$

то из леммы 2 получаем оценку

$$
\left\|\int_{0}^{t} \mathscr{G}(t-\tau) f\left(v_{m-1}(\tau), h_{m-1}(\tau)\right) d \tau\right\|_{\mathbf{X}} \leqslant C \varepsilon^{1+\sigma}
$$

откуда следует, что

$$
\left\|v_{m}\right\|_{\mathbf{x}} \leqslant C \varepsilon, \quad\left\|v_{m}(t)-\mathscr{G}(t) u_{0}\right\|_{\mathbf{L}^{1}} \leqslant C \varepsilon^{1+\sigma}
$$

Для доказательства третьей оценки в (70) мы воспользуемся следующей леммой, в которой вычислена эволюция среднего значения нелинейности в уравнении (1) в субкритическом случае. 
ЛЕмма 8. Предположими, что $u_{0} \in \mathbf{L}^{\infty}\left(\mathbb{R}^{n}\right) \cap \mathbf{L}^{1, a}\left(\mathbb{R}^{n}\right)$ и норма $\left\|u_{0}\right\|_{\mathbf{L}^{\infty}}+$ $\left\|u_{0}\right\|_{\mathbf{L}^{1, a}}=\varepsilon$ достаточно мала. Кроме того, пусть

$$
\theta=\int_{\mathbb{R}^{n}} u_{0}(x) d x \geqslant C \varepsilon>0
$$

Допустим, что функиия $v(t, x)$ удовлетворяет оценкам

$$
\begin{gathered}
\|v\|_{\mathbf{L}^{\infty}} \leqslant C \varepsilon\langle t\rangle^{-\frac{1}{\sigma}}, \quad\|v\|_{\mathbf{L}^{1}} \leqslant C \varepsilon \\
\left\|v(t)-\mathscr{G}(t) u_{0}\right\|_{\mathbf{L}^{1}} \leqslant C \varepsilon^{1+\sigma}
\end{gathered}
$$

для всех $t>0$. Тогда имеет место неравенство

$$
\begin{gathered}
\left.\left|1-\frac{\sigma \lambda}{\theta} \int_{0}^{t} d \tau \int_{\mathbb{R}^{n}}\right| v\right|^{\sigma} v(\tau, x) d x-h_{1}(t) \mid \\
\leqslant C \varepsilon^{\sigma} h_{1}(t)+C \varepsilon^{\sigma} t^{1-\frac{\sigma n}{2}-\frac{a}{2}}
\end{gathered}
$$

для всеx $t>0$.

ДоКАЗАТЕЛЬСтво. В силу леммы 1 получим

$$
\left\|\mathscr{G}(t) u_{0}-\theta G_{0}(t, x)\right\|_{\mathbf{L}^{1}} \leqslant C \varepsilon\langle t\rangle^{-\frac{a}{2}}
$$

для всех $t>0$, откуда находим

$$
\begin{aligned}
&\left\||v|^{\sigma} v-|\theta|^{\sigma} \theta\left(G_{0}(t, x)\right)^{\sigma+1}\right\|_{\mathbf{L}^{1}} \\
& \leqslant C\left(\left\|v(t)-\mathscr{G}(t) u_{0}\right\|_{\mathbf{L}^{1}}+\left\|\mathscr{G}(t) u_{0}-\theta G_{0}(t, x)\right\|_{\mathbf{L}^{1}}\right) \\
& \quad \times\left(\|v\|_{\mathbf{L}^{\infty}}^{\sigma}+\left\|\mathscr{G}_{0}\right\|_{\mathbf{L}^{\infty}}^{\sigma}+|\theta|^{\sigma}\left\|G_{0}\right\|_{\mathbf{L}^{\infty}}^{\sigma}\right) \\
& \leqslant C \varepsilon^{1+\sigma} t^{-\frac{n \sigma}{2}}\left(\varepsilon^{\sigma}+\langle t\rangle^{-\frac{a}{2}}\right)
\end{aligned}
$$

для всех $t>0$. Поскольку

$$
t^{\frac{n}{2} \sigma} \int_{\mathbb{R}^{n}}\left(G_{0}(t, x)\right)^{\sigma+1} d x=(4 \pi \alpha)^{-\frac{n}{2} \sigma}(1+\sigma)^{-\frac{n}{2}}=\frac{\eta}{\sigma \lambda},
$$

получаем

$$
\begin{aligned}
& \left.\left|\int_{\mathbb{R}^{n}}\right| v\right|^{\sigma} v(t, x) d x-|\theta|^{\sigma} \theta t^{-\frac{\sigma}{2} n} \frac{\eta}{\sigma \lambda} \mid \\
& \quad \leqslant C\left\||v|^{\sigma} v-|\theta|^{\sigma} \theta\left(G_{0}(t, x)\right)^{\sigma+1}\right\|_{\mathbf{L}^{1}} \\
& \quad \leqslant C \varepsilon^{1+\sigma} t^{-\frac{n \sigma}{2}}\left(\varepsilon^{\sigma}+\langle t\rangle^{-\frac{a}{2}}\right)
\end{aligned}
$$


для всех $t>0$, где $0<\sigma<\frac{2}{n}$. Следовательно, справедливо неравенство

$$
\begin{aligned}
\mid 1 & -\frac{\sigma \lambda}{\theta} \int_{0}^{t} d \tau \int_{\mathbb{R}^{n}}|v|^{\sigma} v(\tau, x) d x-h_{1}(t) \mid \\
& =\left.\left|\frac{\sigma \lambda}{\theta} \int_{0}^{t} d \tau \int_{\mathbb{R}^{n}}\right| v\right|^{\sigma} v(\tau, x) d x-|\theta|^{\sigma} \eta\left(1-\frac{n}{2} \sigma\right)^{-1} t^{1-\frac{\sigma}{2} n} \mid \\
& \leqslant \frac{C \varepsilon^{2 \sigma}}{2-\sigma n} t^{1-\frac{\sigma}{2} n}+C \varepsilon^{\sigma} t^{1-\frac{\sigma n}{2}-\frac{a}{2}} \leqslant \varepsilon^{\sigma} h_{1}(t)+C \varepsilon^{\sigma} t^{1-\frac{\sigma n}{2}-\frac{a}{2}}
\end{aligned}
$$

для всех $t>0$, откуда вытекает (73). Лемма доказана.

В силу (72) мы можем применить лемму 8 к функции

$$
h_{m}(t)=1-\frac{\sigma \lambda}{\theta} \int_{0}^{t} d \tau \int_{\mathbb{R}^{n}}\left|v_{m-1}\right|^{\sigma} v_{m-1} d x
$$

откуда видим, что

$$
\left|h_{m}(t)-h_{1}(t)\right| \leqslant C \varepsilon^{\sigma} h_{1}(t)
$$

для всех $t>0$. Таким образом, по индукции оценки (70) доказаны для всех $m \geqslant 1$. Точно так же по индукции доказываются неравенства

$$
\begin{aligned}
\left\|v_{m}-v_{m-1}\right\| \mathbf{x} \leqslant & \frac{1}{4}\left\|v_{m-1}-v_{m-2}\right\| \mathbf{x} \\
\sup _{t>0} h_{1}^{-1}(t)\left|h_{m}(t)-h_{m-1}(t)\right| \leqslant & \frac{1}{4}\left\|v_{m-1}-v_{m-2}\right\| \mathbf{x} \\
& +\frac{1}{4} \sup _{t>0} h_{1}^{-1}(t)\left|h_{m-1}(t)-h_{m-2}(t)\right|
\end{aligned}
$$

для всех $m>2$. Следовательно, переходя к пределу в пространстве $\mathbf{X}$ :

$$
\lim _{m \rightarrow \infty} v_{m}(t, x)=v(t, x) \quad \text { и } \quad \lim _{m \rightarrow \infty} h_{m}(t)=h(t),
$$

получаем единственные решения $v(t, x) \in \mathbf{X}, h(t)=e^{\sigma \varphi(t)} \in \mathbf{C}(0, \infty)$, удовлетворяющие уравнениям

$$
\begin{gathered}
v(t)=\mathscr{G}(t) u_{0}+\int_{0}^{t} \mathscr{G}(t-\tau) f(v(\tau), h(\tau)) d \tau \\
h(t)=1-\frac{\sigma \lambda}{\theta} \int_{0}^{t} d \tau \int_{\mathbb{R}^{n}}|v|^{\sigma} v d x
\end{gathered}
$$

и оценкам

$$
\begin{gathered}
\left\|v(t)-\mathscr{G}(t) u_{0}\right\|_{\mathbf{L}^{1}} \leqslant C \varepsilon^{1+\sigma}, \\
\left|h(t)-h_{1}(t)\right| \leqslant C \varepsilon^{\sigma} h_{1}(t) .
\end{gathered}
$$


Также применяя оценку (71) к уравнениям (74), находим

$$
\left\|v(t)-\mathscr{G}(t) u_{0}\right\|_{\mathbf{L}} \leqslant C \varepsilon^{1+\sigma}\langle t\rangle^{-\frac{n}{2}}
$$

для всех $t>0$. Теперь по формулам $u(t, x)=e^{-\varphi(t)} v(t, x)=h^{-\frac{1}{\sigma}}(t) v(t, x)$ получаем оценку

$$
\begin{aligned}
& \left\|u(t)-\theta t^{-\frac{n}{2}} \widetilde{G}\left(t^{-\frac{1}{2}}(\cdot)\right) e^{-\varphi(t)}\right\|_{\mathbf{L}^{\infty}} \\
& \quad \leqslant\left\|u(t)-\left(\mathscr{G}(t) u_{0}\right) e^{-\varphi(t)}\right\|_{\mathbf{L}^{\infty}}+\left\|\left(\mathscr{G}(t) u_{0}-\theta t^{-\frac{n}{2}} \widetilde{G}\left(t^{-\frac{1}{2}}(\cdot)\right)\right) e^{-\varphi(t)}\right\|_{\mathbf{L}^{\infty}} \\
& \quad \leqslant C \varepsilon^{1+\sigma}\langle t\rangle^{-\frac{1}{\sigma}}
\end{aligned}
$$

где мы воспользовались неравенствами

$$
\left\|\left(\mathscr{G}(t) u_{0}-\theta t^{-\frac{n}{2}} \widetilde{G}\left(t^{-\frac{1}{2}}(\cdot)\right)\right) e^{-\varphi(t)}\right\|_{\mathbf{L}^{\infty}} \leqslant C t^{-\frac{1}{\sigma}-\frac{a}{2}}\left\|u_{0}\right\|_{\mathbf{L}^{1, a}}
$$

и (76). Также из (75) имеем

$$
\begin{aligned}
& \left\|\theta t^{-\frac{n}{2}} \widetilde{G}\left(t^{-\frac{1}{2}}(\cdot)\right) h^{-\frac{1}{\sigma}}(t)-\theta t^{-\frac{n}{2}} \widetilde{G}\left(t^{-\frac{1}{2}}(\cdot)\right) h_{1}^{-\frac{1}{\sigma}}(t)\right\|_{\mathbf{L}^{\infty}} \\
& \quad \leqslant C \varepsilon t^{-\frac{n}{2}} h_{1}^{-1-\frac{1}{\sigma}}(t)\left|h(t)-h_{1}(t)\right|,
\end{aligned}
$$

откуда ввиду (77) следует, что

$$
\left\|u(t)-\theta t^{-\frac{n}{2}} \widetilde{G}\left(t^{-\frac{1}{2}}(\cdot)\right) h_{1}^{-\frac{1}{\sigma}}(t)\right\|_{\mathbf{L}^{\infty}} \leqslant C \varepsilon^{1+\sigma}\langle t\rangle^{-\frac{1}{\sigma}} .
$$

Поэтому

$$
\|u(t)\|_{\mathbf{L}^{\infty}} \leqslant \theta t^{-\frac{n}{2}} h_{1}^{-\frac{1}{\sigma}}(t)\left\|\widetilde{G}\left(t^{-\frac{1}{2}}(\cdot)\right)\right\|_{\mathbf{L}^{\infty}}+C \varepsilon^{1+\sigma}\langle t\rangle^{-\frac{1}{\sigma}} \leqslant C t^{-\frac{1}{\sigma}} .
$$

Таким образом, оценка (64) установлена.

Перейдем теперь к вычислению асимптотики решения. Сначала докажем существование решения интегрального уравнения

$$
V(\xi)=V_{1}(\xi)-\frac{1}{\beta} \int_{0}^{1} \frac{d z}{z(1-z)^{\frac{n}{2}}} \int_{\mathbb{R}^{n}} \widetilde{G}\left(\frac{\xi-y z^{\frac{1}{2}}}{(1-z)^{\frac{1}{2}}}\right) F(y) d y,
$$

где $V_{1}(\xi)=\widetilde{G}(\xi)$ и

$$
\begin{gathered}
F(y)=V^{1+\sigma}(y)-V(y) \int_{\mathbb{R}^{n}} V^{\sigma+1}(\xi) d \xi, \\
\beta=\frac{\sigma}{1-\frac{n}{2} \sigma} \int_{\mathbb{R}^{n}} V^{\sigma+1}(\xi) d \xi .
\end{gathered}
$$

Для доказательства сушествования решения интегрального уравнения (78) нам понадобится следующая лемма. 
Лемма 9. Пусть $F(x)$ имеет нулевое среднее значение $\int_{\mathbb{R}^{n}} F(x) d x=0 u$ для некоторого $a, 0<a \leqslant 1$, норма $\|F\|_{\mathbf{L}^{\infty, a}}+\|F\|_{\mathbf{L}^{1, a}}$ ограничена. Тогда имеют место следующие неравенства:

$$
\begin{aligned}
& \left\|\int_{0}^{1} \frac{d z}{z(1-z)^{\frac{n}{2}}} \int_{\mathbb{R}^{n}} \widetilde{G}\left(\left((\cdot)-y z^{\frac{1}{2}}\right)(1-z)^{-\frac{1}{2}}\right) F(y) d y\right\|_{\mathbf{L}^{p, a}} \\
& \leqslant C\|F\|_{\mathbf{L}^{1, a}}+C\|F\|_{\mathbf{L}^{p, a}} \\
& \left\|\int_{0}^{1} \frac{d z}{(1-z)^{\frac{n}{2}}}\left(\frac{1}{z}-\frac{t}{\langle t z\rangle}\right) \int_{\mathbb{R}^{n}} \widetilde{G}\left(\left((\cdot)-y z^{\frac{1}{2}}\right)(1-z)^{-\frac{1}{2}}\right) F(y) d y\right\|_{\mathbf{L}^{p, a}} \\
& \quad \leqslant C\langle t\rangle^{-\frac{a}{2}}\left(\|F\|_{\mathbf{L}^{1, a}}+\|F\|_{\mathbf{L}^{p, a}}\right)
\end{aligned}
$$

для всех $t>0$, әде $1 \leqslant p \leqslant \infty$.

ДокАЗАТЕЛЬСтво. С помощью неравенства Юнга для сверток получаем

$$
\begin{aligned}
& \left\|\int_{\mathbb{R}^{n}} \widetilde{G}\left(\left((\cdot)-y z^{\frac{1}{2}}\right)(1-z)^{-\frac{1}{2}}\right) F(y) d y\right\|_{\mathbf{L}^{p, a}} \\
& \leqslant\left\|\int_{\mathbb{R}^{n}}\left\langle(\cdot)-y z^{\frac{1}{2}}\right\rangle^{a} \widetilde{G}\left(\left((\cdot)-y z^{\frac{1}{2}}\right)(1-z)^{-\frac{1}{2}}\right)|F(y)| d y\right\|_{\mathbf{L}^{p}} \\
& \quad+\left\|\int_{\mathbb{R}^{n}} \widetilde{G}\left(\left((\cdot)-y z^{\frac{1}{2}}\right)(1-z)^{-\frac{1}{2}}\right)\langle y\rangle^{a}|F(y)| d y\right\|_{\mathbf{L}^{p}} \\
& \quad \leqslant\left\|\widetilde{G}\left((\cdot)(1-z)^{-\frac{1}{2}}\right)\right\|_{\mathbf{L}^{1, a}}\|F\|_{\mathbf{L}^{p}}+\left\|\widetilde{G}\left((\cdot)(1-z)^{-\frac{1}{2}}\right)\right\|_{\mathbf{L}^{1}}\|F\|_{\mathbf{L}^{p, a}} \\
& \leqslant(1-z)^{\frac{n}{2}}\|F\|_{\mathbf{L}^{p, a}}
\end{aligned}
$$

для всех $z \in\left[\frac{1}{2}, 1\right]$, поскольку

$$
\left\|\int_{\mathbb{R}^{n}} \varphi\left((\cdot)-y z^{\frac{1}{2}}\right) F(y) d y\right\|_{\mathbf{L}^{p}} \leqslant C\|\varphi\|_{\mathbf{L}^{1}}\|F\|_{\mathbf{L}^{p}}
$$

и, заменяя переменную интегрирования $y=x(1-z)^{-\frac{1}{2}}$, находим

$$
\begin{aligned}
\left\|\widetilde{G}\left((\cdot)(1-z)^{-\frac{1}{2}}\right)\right\|_{\mathbf{L}^{1}} & =\int_{\mathbb{R}^{n}} \widetilde{G}\left(x(1-z)^{-\frac{1}{2}}\right) d x \\
& =(1-z)^{\frac{n}{2}} \int_{\mathbb{R}^{n}} \widetilde{G}(y) d y \leqslant C(1-z)^{\frac{n}{2}}
\end{aligned}
$$

Следовательно, имеем

$$
\begin{aligned}
& \left\|\int_{\frac{1}{2}}^{1} \frac{1}{z(1-z)^{\frac{n}{2}}} \int_{\mathbb{R}^{n}} \widetilde{G}\left(\left((\cdot)-y z^{\frac{1}{2}}\right)(1-z)^{-\frac{1}{2}}\right) F(y) d y d z\right\|_{\mathbf{L}^{p, a}} \\
& \quad \leqslant C \int_{\frac{1}{2}}^{1}(1-z)^{-\frac{n}{2}}\left\|\int_{\mathbb{R}^{n}} \widetilde{G}\left(\left((\cdot)-y z^{\frac{1}{2}}\right)(1-z)^{-\frac{1}{2}}\right) F(y) d y\right\|_{\mathbf{L}^{p, a}} d z \\
& \quad \leqslant C\|F\|_{\mathbf{L}^{p, a}}
\end{aligned}
$$


ЗАДАЧА КОШИ ДЛЯ УРАВНЕНИЯ ТИПА СОБОЛЕВА

103

$$
\begin{aligned}
& \left\|\int_{\frac{1}{2}}^{1} \frac{d z}{(1-z)^{\frac{n}{2}}}\left(\frac{1}{z}-\frac{t}{\langle t z\rangle}\right) \int_{\mathbb{R}^{n}} \widetilde{G}\left(\frac{(\cdot)-y z^{\frac{1}{2}}}{(1-z)^{\frac{1}{2}}}\right) F(y) d y\right\|_{\mathbf{L}^{p, a}} \\
& \quad \leqslant C\langle t\rangle^{-1} \int_{\frac{1}{2}}^{1}(1-z)^{-\frac{n}{2}}\left\|\int_{\mathbb{R}^{n}} \widetilde{G}\left(\frac{(\cdot)-y z^{\frac{1}{2}}}{(1-z)^{\frac{1}{2}}}\right) F(y) d y\right\|_{\mathbf{L}^{p, a}} d z \\
& \quad \leqslant C\langle t\rangle^{-1}\|F\|_{\mathbf{L}^{p, a}}
\end{aligned}
$$

где $1 \leqslant p \leqslant \infty$.

Ввиду условия $\int_{\mathbb{R}^{n}} F(y) d y=0$ запишем

$$
\begin{aligned}
& \left\|\int_{\mathbb{R}^{n}} \widetilde{G}\left(\left((\cdot)-y z^{\frac{1}{2}}\right)(1-z)^{-\frac{1}{2}}\right) F(y) d y\right\|_{\mathbf{L}^{p, a}} \\
& =\|\langle\cdot\rangle^{a} \int_{\mathbb{R}^{n}}\left(\widetilde{G}\left(\left((\cdot)-y z^{\frac{1}{2}}\right)(1-z)^{-\frac{1}{2}}\right)\right. \\
& \left.-\widetilde{G}\left((\cdot)(1-z)^{-\frac{1}{2}}\right)\right) F(y) d y \|_{\mathbf{L}^{p}} \\
& \leqslant\left\|\int_{\mathbb{R}^{n}}\left(\left(\langle\cdot\rangle^{a}-\left\langle(\cdot)-y z^{\frac{1}{2}}\right\rangle^{a}\right) \widetilde{G}\left(\frac{(\cdot)-y z^{\frac{1}{2}}}{(1-z)^{\frac{1}{2}}}\right)\right) F(y) d y\right\|_{\mathbf{L}^{p}} \\
& +\| \int_{\mathbb{R}^{n}}\left(\left\langle(\cdot)-y z^{\frac{1}{2}}\right\rangle^{a} \widetilde{G}\left(\left((\cdot)-y z^{\frac{1}{2}}\right)(1-z)^{-\frac{1}{2}}\right)\right. \\
& \left.-\langle\cdot\rangle^{a} \widetilde{G}\left((\cdot)(1-z)^{-\frac{1}{2}}\right)\right) F(y) d y \|_{\mathbf{L}^{p}} \\
& \leqslant C z^{\frac{a}{2}}\left\|\int_{\mathbb{R}^{n}} \widetilde{G}\left(\left((\cdot)-y z^{\frac{1}{2}}\right)(1-z)^{-\frac{1}{2}}\right)\langle y\rangle^{a}|F(y)| d y\right\|_{\mathbf{L}^{p}} \\
& +C z^{\frac{a}{2}} \| \int_{\mathbb{R}^{n}}\left(\widetilde{G}\left(\left((\cdot)-y z^{\frac{1}{2}}\right)(1-z)^{-\frac{1}{2}}\right)\right. \\
& \left.+\widetilde{G}\left((\cdot)(1-z)^{-\frac{1}{2}}\right)\right)\langle y\rangle^{a}|F(y)| d y \|_{\mathbf{L}^{p}} \\
& \leqslant C z^{\frac{a}{2}}\|F\|_{\mathbf{L}^{1, a}}\left\|\widetilde{G}\left((\cdot)(1-z)^{-\frac{1}{2}}\right)\right\|_{\mathbf{L}^{p}} \leqslant C z^{\frac{a}{2}}\|F\|_{\mathbf{L}^{1, a}} \\
& \left\|\int_{\mathbb{R}^{n}} \varphi\left((\cdot)-y z^{\frac{1}{2}}\right) F(y) d y\right\|_{\mathbf{L}^{p}} \leqslant C\left\|_{\varphi}\right\|_{\mathbf{L}^{p}}\|F\|_{\mathbf{L}^{1}} .
\end{aligned}
$$

для всех $z \in\left(0, \frac{1}{2}\right)$, поскольку

Таким образом, находим

$$
\begin{aligned}
& \left\|\int_{0}^{\frac{1}{2}} \frac{1}{z(1-z)^{\frac{n}{2}}} \int_{\mathbb{R}^{n}} \widetilde{G}\left(\left((\cdot)-y z^{\frac{1}{2}}\right)(1-z)^{-\frac{1}{2}}\right) F(y) d y d z\right\|_{\mathbf{L}^{p, a}} \\
& \quad \leqslant \int_{0}^{\frac{1}{2}} \frac{d z}{z(1-z)^{\frac{n}{2}}}\left\|\int_{\mathbb{R}^{n}} \widetilde{G}\left(\left((\cdot)-y z^{\frac{1}{2}}\right)(1-z)^{-\frac{1}{2}}\right) F(y) d y\right\|_{\mathbf{L}^{p, a}} \\
& \quad \leqslant C \int_{0}^{\frac{1}{2}} \frac{d z}{z^{1-\frac{a}{2}}(1-z)^{\frac{n}{2}}}\|F\|_{\mathbf{L}^{1, a}} \leqslant C\|F\|_{\mathbf{L}^{1, a}},
\end{aligned}
$$




$$
\begin{aligned}
& \left\|\int_{0}^{\frac{1}{2}} \frac{d z}{(1-z)^{\frac{n}{2}}}\left(\frac{1}{z}-\frac{t}{\langle t z\rangle}\right) \int_{\mathbb{R}^{n}} \widetilde{G}\left(\frac{(\cdot)-y z^{\frac{1}{2}}}{(1-z)^{\frac{1}{2}}}\right) F(y) d y\right\|_{\mathbf{L}^{p, a}} \\
& \quad \leqslant \int_{0}^{\frac{1}{2}} \frac{d z}{(1-z)^{\frac{n}{2}}}\left(\frac{1}{z}-\frac{t}{\langle t z\rangle}\right)\left\|\int_{\mathbb{R}^{n}} \widetilde{G}\left(\frac{(\cdot)-y z^{\frac{1}{2}}}{(1-z)^{\frac{1}{2}}}\right) F(y) d y\right\|_{\mathbf{L}^{p, a}} \\
& \quad \leqslant C \int_{0}^{\frac{1}{2}} \frac{z^{\frac{a}{2}}}{(1-z)^{\frac{n}{2}}}\left(\frac{1}{z}-\frac{t}{\langle t z\rangle}\right) d z\|F\|_{\mathbf{L}^{1, a}} \leqslant C\langle t\rangle^{-\frac{a}{\alpha}}\|F\|_{\mathbf{L}^{1, a}},
\end{aligned}
$$

где $1 \leqslant p \leqslant \infty$. Теперь, используя оценки $(79)-(82)$, приходим к результату леммы. Лемма доказана.

Зададим последовательные приближения $V_{k+1}=\mathscr{Q}\left(V_{k}\right)$ для всех $k=1,2, \ldots$, где

$$
\begin{gathered}
\mathscr{Q}\left(V_{k}\right)(\xi)=V_{1}(\xi)-\frac{1}{\beta_{k}} \int_{0}^{1} \frac{d z}{z(1-z)^{\frac{n}{2}}} \int_{\mathbb{R}^{n}} \widetilde{G}\left(\frac{\xi-y z^{\frac{1}{2}}}{(1-z)^{\frac{1}{2}}}\right) F_{k}(y) d y \\
F_{k}(y)=V_{k}^{\sigma+1}(y)-V_{k}(y) \int_{\mathbb{R}^{n}} V_{k}^{\sigma+1}(\xi) d \xi \\
\beta_{k}=\frac{\sigma}{1-\frac{n}{2} \sigma} \int_{\mathbb{R}^{n}} V_{k}^{\sigma+1}(\xi) d \xi
\end{gathered}
$$

По индукции, с помощью леммы 9 , докажем оценки

$$
\begin{aligned}
& \sup _{1 \leqslant p \leqslant \infty}\left\|V_{k+1}-V_{1}\right\|_{\mathbf{L}^{p, a}} \leqslant C \varepsilon, \quad \sup _{1 \leqslant p \leqslant \infty}\left\|V_{k}\right\|_{\mathbf{L}^{p, a}} \leqslant C, \quad \beta_{k} \geqslant C \varepsilon^{-1} \\
& \sup _{1 \leqslant p \leqslant \infty}\left\|V_{k+1}-V_{k}\right\|_{\mathbf{L}^{p}} \leqslant \frac{1}{2} \sup _{1 \leqslant p \leqslant \infty}\left\|V_{k}-V_{k-1}\right\|_{\mathbf{L}^{p}}
\end{aligned}
$$

для всех $k \geqslant 1$. Чтобы применить лемму 9 , надо показать, что

$$
\int_{\mathbb{R}^{n}} F_{k}(y) d y=0, \quad \int_{\mathbb{R}^{n}} V_{k}(y) d y=1 .
$$

Поскольку $\int_{\mathbb{R}^{n}} V_{1}(y) d y=1$, то по определению $F_{1}(y)$ имеем

$$
\begin{aligned}
\int_{\mathbb{R}^{n}} F_{1}(y) d y & =\int_{\mathbb{R}^{n}}\left(V_{1}^{\sigma+1}(y)-V_{1}(y) \int_{\mathbb{R}^{n}} V_{1}^{\sigma+1}(\xi) d \xi\right) d y \\
& =\int_{\mathbb{R}^{n}} V_{1}^{\sigma+1}(y) d y-\int_{\mathbb{R}^{n}} V_{1}^{\sigma+1}(\xi) d \xi=0
\end{aligned}
$$

так что равенства (85) справедливы при $k=1$. Предположим, по индукции, что равенства (85) имеют место для некоторого $k$. Тогда из рекуррентных соотношений, определяющих $V_{k+1}(\xi)$, найдем

$$
\int_{\mathbb{R}^{n}} V_{k+1}(\xi) d \xi=1-\frac{1}{\beta_{k}} \int_{0}^{1} \frac{d z}{z(1-z)^{\frac{n}{2}}} \int_{\mathbb{R}^{n}} d y F_{k}(y) \int_{\mathbb{R}^{n}} \widetilde{G}\left(\frac{\xi-y z^{\frac{1}{2}}}{(1-z)^{\frac{1}{2}}}\right) d \xi=1
$$


откуда, как и выше, получаем, что $\int_{\mathbb{R}^{n}} F_{k+1}(y) d y=0$. Таким образом, равенства (85) доказаны для всех $k$. Применив лемму 9 , получим

$$
\begin{aligned}
\sup _{1 \leqslant p \leqslant \infty}\left\|V_{k+1}-V_{1}\right\|_{\mathbf{L}^{p, a}} & \\
= & \frac{C}{\beta_{k}} \sup _{1 \leqslant p \leqslant \infty}\left\|\int_{0}^{1} \frac{d z}{z(1-z)^{\frac{n}{2}}} \int_{\mathbb{R}^{n}} \widetilde{G}\left(\frac{(\cdot)-y z^{\frac{1}{2}}}{(1-z)^{\frac{1}{2}}}\right) F_{k}(y) d y\right\|_{\mathbf{L}^{p, a}} \\
& \leqslant \frac{C}{\beta_{k}} \sup _{1 \leqslant p \leqslant \infty}\left\|\left(V_{k}^{\sigma+1}(\cdot)-V_{k}(\cdot) \int_{\mathbb{R}^{n}} V_{k}^{\sigma+1}(\xi) d \xi\right)\right\|_{\mathbf{L}^{p, a}} \\
& \left.\leqslant \frac{C}{\beta_{k}} \sup _{1 \leqslant p \leqslant \infty}\left\|V_{k}\right\|_{\mathbf{L}^{p, a}}\left(\sup _{1 \leqslant p \leqslant \infty}\left\|V_{k}\right\|_{\mathbf{L}^{p}}\right)^{\sigma}+\left(\sup _{1 \leqslant p \leqslant \infty}\left\|V_{k}\right\|_{\mathbf{L}^{p}}\right)^{\sigma+1}\right) \leqslant C \varepsilon,
\end{aligned}
$$

так как $\sigma$ близко к $\frac{2}{n}$, поэтому $\beta_{k}$ можно считать достаточно большим. Следовательно, оценки (83) имеют место для всех $k$. Аналогично доказывается неравенство (84) для всех $k \geqslant 1$. Отсюда видим, что $\mathscr{Q}$ является сжимающим отображением, значит, сушествует единственное решение $V(\xi)$ интегрального уравнения (78).

Перейдем к доказательству асимптотики решения $v$. Установим оценку для последовательных приближений

$$
\left\|v_{k}(t)-t^{-\frac{n}{2}} \theta V_{k}\left((\cdot) t^{-\frac{1}{2}}\right)\right\|_{\mathbf{L}^{p, b}} \leqslant C\langle t\rangle^{\frac{b}{2}} t^{-\frac{n}{2}\left(1-\frac{1}{p}\right)-\gamma}
$$

для всех $k \geqslant 1, t>0, b \in[0, a], 1 \leqslant p \leqslant \infty$, где $\gamma=\frac{1}{2} \min \left(a, 1-\frac{n}{2} \sigma\right)$. Проведем индукцию по $k \geqslant 1$. При $k=1$ в силу леммы 1 получим

$$
\begin{aligned}
\left\|v_{1}(t)-t^{-\frac{n}{2}} \theta V_{1}\left((\cdot) t^{-\frac{1}{2}}\right)\right\|_{\mathbf{L}^{p, b}} & =\left\|\mathscr{G}(t) u_{0}-t^{-\frac{n}{2}} \theta \widetilde{G}\left((\cdot) t^{-\frac{1}{2}}\right)\right\|_{\mathbf{L}^{p, b}} \\
& \leqslant C\langle t\rangle^{\frac{b}{2}} t^{-\frac{n}{2}\left(1-\frac{1}{p}\right)}\langle t\rangle^{-\frac{a}{2}}
\end{aligned}
$$

Теперь по индукции предположим, что оценка (86) справедлива для некоторого номера $k$ :

$$
\left\|v_{k}(t)-t^{-\frac{n}{2}} \theta V_{k}\left((\cdot) t^{-\frac{1}{2}}\right)\right\|_{\mathbf{L}^{p, b}} \leqslant C\langle t\rangle^{\frac{b}{2}} t^{-\frac{n}{2}\left(1-\frac{1}{p}\right)-\gamma}
$$

для всех $t>0, b \in[0, a], 1 \leqslant p \leqslant \infty$, и докажем (86) для следуюшего номера $k+1$. Тогда, поскольку

$$
\beta_{k}=\frac{\sigma}{1-\frac{n}{2} \sigma} \int_{\mathbb{R}^{n}} V_{k}^{\sigma+1}(\xi) d \xi,
$$

из неравенства (88) следует, что

$$
\begin{aligned}
\left.\left|h_{k}(t)-\right| \theta\right|^{\sigma} \beta_{k} t^{1-\frac{n}{2} \sigma}|=| 1-\frac{\sigma \lambda}{\theta} \int_{0}^{t} \int_{\mathbb{R}^{n}}\left|v_{k}\right|^{\sigma} v_{k}(\tau, x) d x d \tau \\
\quad-t^{1-\frac{n}{2} \sigma} \frac{\sigma \theta^{\sigma}}{1-\frac{n}{2} \sigma} \int_{\mathbb{R}^{n}} V_{k}^{1+\sigma}(\xi) d \xi \mid \\
\leqslant 1+\left.\frac{C}{\theta} \int_{0}^{t} \int_{\mathbb{R}^{n}}|| v_{k}\right|^{\sigma} v_{k}(\tau, x)-|\theta|^{\sigma} \theta \tau^{-\frac{n}{2} \sigma-\frac{n}{2}} V_{k}^{1+\sigma}\left(x \tau^{-\frac{1}{2}}\right) \mid d x d \tau
\end{aligned}
$$




$$
\begin{aligned}
& \leqslant 1+\frac{C}{\theta} \int_{0}^{t}\left(\left\|v_{k}\right\|_{\mathbf{L}^{\infty}}+\theta \tau^{-\frac{n}{2}}\left\|V_{k}\right\|_{\mathbf{L}^{\infty}}\right)^{\sigma}\left\|v_{k}(\tau, \cdot)-\theta \tau^{-\frac{n}{2}} V_{k}\left((\cdot) \tau^{-\frac{1}{2}}\right)\right\|_{\mathbf{L}^{1}} d \tau \\
& \leqslant 1+\frac{C}{|\theta|} \int_{0}^{t} \tau^{-\frac{n}{2} \sigma-\gamma} d \tau \leqslant 1+C \beta_{k} t^{1-\frac{n}{2} \sigma-\gamma}
\end{aligned}
$$

для всех $t>0,1 \leqslant p \leqslant \infty$. Делая замену переменных $\tau=z t$ и $\xi \tau^{-\frac{1}{2}}=y$, находим

$$
\begin{aligned}
& \frac{1}{\beta_{k}} \int_{0}^{t} \tau^{\frac{n}{2} \sigma-1} \mathscr{G}_{0}(t-\tau) \tau^{-\frac{n}{2} \sigma-\frac{n}{2}} F_{k}\left((\cdot) \tau^{-\frac{1}{2}}\right) d \tau \\
& \quad=\frac{1}{\beta_{k}} \int_{0}^{t} \tau^{-1-\frac{n}{2}}(t-\tau)^{-\frac{n}{2}} d \tau \int_{\mathbb{R}^{n}} \widetilde{G}\left((x-\xi)(t-\tau)^{-\frac{1}{2}}\right) F_{k}\left(\xi \tau^{-\frac{1}{2}}\right) d \xi \\
& \quad=\frac{1}{\beta_{k}} \int_{0}^{1} \frac{d z}{z(1-z)^{\frac{n}{2}}} \int_{\mathbb{R}^{n}} \widetilde{G}\left(\left(x t^{-\frac{1}{2}}-y z^{\frac{1}{2}}\right)(1-z)^{-\frac{1}{2}}\right) F_{k}(y) d y \\
& \quad=t^{-\frac{n}{2}}\left(V_{1}\left(x t^{-\frac{1}{2}}\right)-V_{k}\left(x t^{-\frac{1}{2}}\right)\right),
\end{aligned}
$$

поэтому, обозначив

$$
f_{k}(\tau)=\lambda\left|v_{k}\right|^{\sigma} v_{k}(\tau)-\frac{\lambda v_{k}(\tau)}{\theta} \int_{\mathbb{R}^{n}}\left|v_{k}\right|^{\sigma} v_{k}(\tau) d x
$$

получаем

$$
\begin{aligned}
\langle t\rangle^{-\frac{b}{2}} \| & \theta t^{-\frac{n}{2}} V_{k}\left((\cdot) t^{-\frac{1}{2}}\right)-v_{k}(t) \|_{\mathbf{L}^{p, b}} \\
= & \langle t\rangle^{-\frac{b}{2}}\left\|\theta t^{-\frac{n}{2}} V_{k}\left((\cdot) t^{-\frac{1}{2}}\right)-\mathscr{G}(t) u_{0}+\int_{0}^{t} h^{-1}(\tau) \mathscr{G}(t-\tau) \mathscr{B} f_{k}(\tau) d \tau\right\|_{\mathbf{L}^{p, b}} \\
\leqslant & C\langle t\rangle^{-\frac{b}{2}}\left\|\theta t^{-\frac{n}{2}} V_{1}\left((\cdot) t^{-\frac{1}{2}}\right)-\mathscr{G}(t) u_{0}\right\|_{\mathbf{L}^{p, b}} \\
& +C\langle t\rangle^{-\frac{b}{2}}\left\|\int_{0}^{t}\left(h_{k}^{-1}(\tau)-\frac{1}{\beta_{k}|\theta|^{\sigma}} \tau^{\frac{n}{2} \sigma}\langle\tau\rangle^{-1}\right) \mathscr{G}(t-\tau) \mathscr{B} f_{k}(\tau) d \tau\right\|_{\mathbf{L}^{p, b}} \\
& +\frac{C\langle t\rangle^{-\frac{b}{2}}}{\beta_{k}|\theta|^{\sigma}}\left\|\int_{0}^{t}\left(\mathscr{G}(t-\tau) \mathscr{B}-\mathscr{G}_{0}(t-\tau)\right) f_{k}(\tau) \frac{\tau^{\frac{n}{2} \sigma} d \tau}{\langle\tau\rangle}\right\|_{\mathbf{L}^{p, b}} \\
& +\frac{C\langle t\rangle^{-\frac{b}{2}}}{\beta_{k}|\theta|^{\sigma}}\left\|\int_{0}^{t}\left(\mathscr{G}_{0}(t-\tau)\left(f_{k}(\tau)-\frac{|\theta|^{\sigma} \theta \lambda}{\tau^{\frac{n}{2}(\sigma+1)}} F_{k}\left((\cdot) \tau^{-\frac{1}{2}}\right)\right)\right) \frac{\tau^{\frac{n}{2} \sigma} d \tau}{\langle\tau\rangle}\right\|_{\mathbf{L}^{p, b}} \\
& +\frac{C|\theta \lambda|\langle t\rangle^{-\frac{b}{2}}}{\beta_{k}}\left\|\int_{0}^{t} \mathscr{G}_{0}(t-\tau) F_{k}\left((\cdot) \tau^{-\frac{1}{2}}\right)\left(\frac{1}{\langle\tau\rangle}-\frac{1}{\tau}\right) \tau^{-\frac{n}{2}} d \tau\right\|_{\mathbf{L}^{p, b}} \\
\equiv & I_{1}+I_{2}+I_{3}+I_{4}+I_{5} .
\end{aligned}
$$

Согласно (87) имеем

$$
I_{1} \leqslant C t^{-\frac{n}{2}\left(1-\frac{1}{p}\right)}\langle t\rangle^{-\frac{a}{2}}
$$


Ввиду (89) из леммы 9 получаем

$$
\begin{aligned}
I_{2} \leqslant & C\langle t\rangle^{-\frac{b}{2}}\left\|\int_{0}^{t} \frac{\tau^{\frac{n}{2} \sigma}}{\langle\tau\rangle}\left|\frac{\langle\tau\rangle}{\tau^{\frac{n}{2} \sigma}}-\frac{h_{k}(\tau)}{\beta_{k} \theta^{\sigma}}\right| h_{k}^{-1}(\tau) \mathscr{G}(t-\tau) \mathscr{B} f_{k}(\tau) d \tau\right\|_{\mathbf{L}^{p, b}} \\
\leqslant & C\langle t\rangle^{-\frac{b}{2}} \| \int_{0}^{t} \frac{\tau^{\frac{n}{2} \sigma}}{\langle\tau\rangle}\left(\frac{\langle\tau\rangle}{\tau^{\frac{n}{2} \sigma}}-\tau^{1-\frac{n}{2} \sigma}+\frac{1}{\beta_{k}|\theta|^{\sigma}}+C \tau^{1-\frac{n}{2} \sigma-\gamma}\right) \\
& \times h_{k}^{-1}(\tau)^{\mathscr{G}}(t-\tau) \mathscr{B} f_{k}(\tau) d \tau \|_{\mathbf{L}^{p, b}} \\
\leqslant & C\langle t\rangle^{-\frac{b}{2}}\left\|\int_{0}^{t}\langle\tau\rangle^{-\gamma} h_{k}^{-1}(\tau)^{\mathscr{G}}(t-\tau) \mathscr{B} f_{k}(\tau) d \tau\right\|_{\mathbf{L}^{p, b}} \leqslant C t^{-\frac{n}{2}\left(1-\frac{1}{p}\right)-\gamma}
\end{aligned}
$$

для всех $t>0,1 \leqslant p \leqslant \infty$. Благодаря оценке (71), применяя лемму 1 , находим

$$
\begin{aligned}
I_{3} \leqslant & C\langle t\rangle^{-\frac{b}{2}}\left\|\int_{0}^{t}\left(\mathscr{G}(t-\tau) \mathscr{B}-\mathscr{G}_{0}(t-\tau)\right) f_{k}(\tau) \frac{\tau^{\frac{n}{2} \sigma} d \tau}{\langle\tau\rangle}\right\|_{\mathbf{L}^{p, b}} \\
\leqslant & C\langle t\rangle^{-\frac{b}{2}} \int_{0}^{\frac{t}{2}} \tau^{\frac{n}{2} \sigma}\langle\tau\rangle^{-1}\langle t-\tau\rangle^{-\frac{n}{2}\left(1-\frac{1}{p}\right)-\gamma}\left(\langle\tau\rangle^{\frac{b}{2}}+\langle t-\tau\rangle^{\frac{b}{2}}\right) d \tau \\
& \quad+C\langle t\rangle^{-\frac{b}{2}} \int_{\frac{t}{2}}^{t} \tau^{\frac{n}{2} \sigma}\langle\tau\rangle^{-1-\frac{n}{2}\left(1-\frac{1}{p}\right)}\langle t-\tau\rangle^{-\gamma}\left(\langle\tau\rangle^{\frac{b}{2}}+\langle t-\tau\rangle^{\frac{b}{2}}\right) d \tau \\
& \leqslant C t^{-\frac{n}{2}\left(1-\frac{1}{p}\right)-\gamma}
\end{aligned}
$$

для всех $t>0,1 \leqslant p \leqslant \infty$. Аналогично получаем

$$
\begin{aligned}
I_{4}= & \frac{C\langle t\rangle^{-\frac{b}{2}}}{\beta_{k}|\theta|^{\sigma}}\left\|\int_{0}^{t} \mathscr{G}_{0}(t-\tau)\left(f_{k}(\tau)-\tau^{-\frac{n}{2} \sigma-\frac{n}{2}}|\theta|^{\sigma} \theta \lambda F_{k}\left((\cdot) \tau^{-\frac{1}{2}}\right)\right) \frac{\tau^{\frac{n}{2} \sigma} d \tau}{\langle\tau\rangle}\right\|_{\mathbf{L}^{p, b}} \\
\leqslant & \frac{C}{\beta_{k}|\theta|^{\sigma}} t^{-\frac{n}{2}\left(1-\frac{1}{p}\right)-\gamma} \\
& \quad \times \sup _{t>0} \sup _{1 \leqslant p \leqslant \infty} t^{\frac{n}{2}\left(1+\sigma-\frac{1}{p}\right)+\gamma}\langle t\rangle^{-\frac{b}{2}}\left\|f_{k}(t)-t^{-\frac{n}{2} \sigma-\frac{n}{2}}|\theta|^{\sigma} \theta \lambda F_{k}\left((\cdot) t^{-\frac{1}{2}}\right)\right\|_{\mathbf{L}^{p, b}} \\
\leqslant & \frac{C}{\beta_{k}|\theta|^{\sigma}} t^{-\frac{n}{2}\left(1-\frac{1}{p}\right)-\gamma}\left(\operatorname { s u p } _ { t > 0 } \left(t^{\frac{n}{2}}\left\|v_{k}(t)\right\|_{\left.\left.\mathbf{L}^{\infty}+\theta\left\|V_{k}\right\|_{\mathbf{L}} \boldsymbol{}\right)\right)^{\sigma}}\right.\right. \\
& \quad \times \sup _{t>0} \sup _{1 \leqslant p \leqslant \infty} t^{\frac{n}{2}\left(1-\frac{1}{p}\right)+\gamma}\langle t\rangle^{-\frac{b}{2}}\left\|v_{k}(t)-t^{-\frac{n}{2}} \theta V_{k}\left((\cdot) t^{-\frac{1}{2}}\right)\right\|_{\mathbf{L}^{p, b}} \\
\leqslant & C t^{-\frac{n}{2}\left(1-\frac{1}{p}\right)-\gamma}
\end{aligned}
$$

для всех $t>0,1 \leqslant p \leqslant \infty$. Наконец, заменяя переменные $\tau=z t$ и $\xi \tau^{-\frac{1}{2}}=y$, 
с помошью леммы 9 находим

$$
\begin{aligned}
I_{5}= & \frac{C|\theta \lambda|}{\beta_{k}\langle t\rangle^{\frac{b}{2}}}\left\|\int_{0}^{t} \mathscr{G}_{0}(t-\tau) F_{k}\left((\cdot) \tau^{-\frac{1}{2}}\right)\left(\frac{1}{\langle\tau\rangle}-\frac{1}{\tau}\right) \tau^{-\frac{n}{2}} d \tau\right\|_{\mathbf{L}^{p, b}} \\
= & \frac{C|\theta \lambda|\langle t\rangle^{-\frac{b}{2}}}{\beta_{k}} \| \int_{0}^{t}\left(\frac{1}{\langle\tau\rangle}-\frac{1}{\tau}\right) \tau^{-\frac{n}{2}}(t-\tau)^{-\frac{n}{2}} d \tau \\
& \times \int_{\mathbb{R}^{n}} \widetilde{G}\left(((\cdot)-\xi)(t-\tau)^{-\frac{1}{2}}\right) F_{k}\left(\xi \tau^{-\frac{1}{2}}\right) d \xi \|_{\mathbf{L}^{p, b}} \\
= & C t^{-\frac{n}{2}\left(1-\frac{1}{p}\right)} \| \int_{0}^{1} \frac{d z}{(1-z)^{\frac{n}{2}}}\left(\frac{1}{z}-\frac{t}{\langle t z\rangle}\right) \\
& \times \int_{\mathbb{R}^{n}} \widetilde{G}\left(\left((\cdot)-y z^{\frac{1}{2}}\right)(1-z)^{-\frac{1}{2}}\right) F_{k}(y) d y \|_{\mathbf{L}^{p, b}} \\
\leqslant & C t^{-\frac{n}{2}\left(1-\frac{1}{p}\right)-\gamma}
\end{aligned}
$$

для всех $t>0,1 \leqslant p \leqslant \infty$, откуда следует (86) для всех $t>0$. Взяв $b=0$ в (86) и переходя к пределу при $k \rightarrow \infty$, получаем

$$
\left\|v(t)-t^{-\frac{n}{2}} \theta V\left((\cdot) t^{-\frac{1}{2}}\right)\right\|_{\mathbf{L}^{p}} \leqslant C t^{-\frac{n}{2}\left(1-\frac{1}{p}\right)-\gamma} .
$$

Отсюда в силу (89) и (90) находим асимптотику

$$
\begin{gathered}
v(t)=t^{-\frac{n}{2}} \theta V\left((\cdot) t^{-\frac{1}{2}}\right)+O\left(t^{-\frac{n}{2}-\gamma}\right), \\
h(t)=|\theta|^{\sigma} \beta t^{1-\frac{n}{2} \sigma}\left(1+O\left(t^{-\gamma}\right)\right)
\end{gathered}
$$

при $t \rightarrow \infty$ равномерно по $x \in \mathbb{R}^{n}$ (поскольку решение $v(t, x) \in \mathbf{X}$, вследствие чего оно является непрерывным). Откуда ввиду формулы $u(t, x)=e^{-\varphi(t)} v(t, x)$, принимая во внимание оценку (90), получаем асимптотику $(65)$ решения $u(t, x)$ с константой $A=\beta^{-\frac{1}{\sigma}}$, где

$$
\beta=\frac{\sigma}{1-\frac{n}{2} \sigma} \int_{\mathbb{R}^{n}} V^{\sigma+1}(\xi) d \xi
$$

Доказательство теоремы завершено.

5.2. Большие начальные данные. Здесь мы освободимся от условия малости начальных данных и установим глобальное во времени существование решений задачи Коши (1) с субкритической степенью $\sigma \in\left(0, \frac{2}{n}\right)$ нелинейности. По-прежнему будем предполагать, что $\sigma \in\left(0, \frac{2}{n}\right)$ достаточно близко к $\frac{2}{n}$.

Теорема 6. Пусть $\lambda<0, n=1,2$. Предположим, что начальные данные $u_{0} \in \mathbf{W}_{\infty}^{2}\left(\mathbb{R}^{n}\right) \cap \mathbf{W}_{1}^{2, a}\left(\mathbb{R}^{n}\right), 0<a \leqslant 1$, таковиь, что $\theta=\int_{\mathbb{R}^{n}} u_{0}(x) d x \neq 0$. Предположим также, что $\frac{2}{n}-\varepsilon<\sigma<\frac{2}{n}$, где $\varepsilon>0$ достаточно мало, так что заведомо $\sigma>1$ для $n=1$ и $\sigma>\frac{3}{4}$ для $n=2$. Тогда существует единственное глобальное во времени решение $u(t, x) \in \mathbf{C}\left([0, \infty) ; \mathbf{C}\left(\mathbb{R}^{n}\right) \cap \mathbf{L}^{\infty}\left(\mathbb{R}^{n}\right) \cap \mathbf{L}^{1, a}\left(\mathbb{R}^{n}\right)\right)$ задачи Коши (1). Это решение удовлетворяет оценке убивания (64) и имеет асимптотическое представление (65), (66). 
ДокАЗАТЕЛЬСТво. Как и при доказательстве теоремы 4, с помошью леммы 1 и утверждения 2 находим

$$
\begin{aligned}
\|u(t)\|_{\mathbf{L}^{\infty}} \leqslant & \left\|\mathscr{G}(t) u_{0}\right\|_{\mathbf{L}^{\infty}}+C \int_{\frac{t}{2}}^{t}\langle t-\tau\rangle^{-\frac{n(\sigma+1)}{2(\sigma+2)}}\|u(\tau)\|_{\mathbf{L}^{\sigma+2}}^{\sigma+1} d \tau \\
& +C \int_{0}^{\frac{t}{2}}\langle t-\tau\rangle^{-\frac{n}{2}}\left(\left\||u|^{\sigma} u(\tau)\right\|_{\mathbf{L}^{1}}+\|u(\tau)\|_{\mathbf{L}^{\sigma+2}}^{\sigma+1}\right) d \tau \\
\leqslant & C\langle t\rangle^{-\frac{n}{2}}+C \int_{\frac{t}{2}}^{t}\langle t-\tau\rangle^{-\frac{n(\sigma+1)}{2(\sigma+2)}}\langle\tau\rangle^{-\frac{n(\sigma+1)^{2}}{2(\sigma+2)}} d \tau \\
& +C \int_{0}^{\frac{t}{2}}\langle t-\tau\rangle^{-\frac{n}{2}}\langle\tau\rangle^{-\frac{n}{2} \sigma} d \tau \leqslant C\langle t\rangle^{1-\frac{n}{2} \sigma-\frac{n}{2}}
\end{aligned}
$$

для всех $t>0$. Аналогично,

$$
\begin{aligned}
\|\Delta u(t)\|_{\mathbf{L}} \leqslant \leqslant & C\langle t\rangle^{-1-\frac{n}{2}}+\int_{0}^{t}\left\|\Delta \mathscr{G}(t-\tau) \mathscr{B}|u|^{\sigma} u(\tau)\right\|_{\mathbf{L}^{\infty}} d \tau \\
\leqslant & C\langle t\rangle^{-1-\frac{n}{2}}+C \int_{0}^{\frac{t}{2}}\langle t-\tau\rangle^{-1-\frac{n}{2}}\|u(\tau)\|_{\mathbf{L}^{\sigma+1}}^{\sigma+1} d \tau \\
& +C \int_{\frac{t}{2}}^{t}\langle t-\tau\rangle^{-1}\|u(\tau)\|_{\mathbf{L}^{\infty}}^{\sigma+1} d \tau \\
\leqslant & C\langle t\rangle^{-1-\frac{n}{2}}+C \int_{0}^{\frac{t}{2}}\langle t-\tau\rangle^{-1-\frac{n}{2}}\langle\tau\rangle^{-\frac{n}{2} \sigma} d \tau \\
& +C \int_{\frac{t}{2}}^{t}\langle t-\tau\rangle^{-1}\langle t\rangle^{\left(1-\frac{n}{2} \sigma-\frac{n}{2}\right)(\sigma+1)} d \tau \\
\leqslant & C\langle t\rangle^{\left(1-\frac{n}{2} \sigma-\frac{n}{2}\right)(\sigma+1)}
\end{aligned}
$$

для всех $t>0$. Обозначим $f(t, x)=\Delta u_{t}$. Как и при доказательстве теоремы 4 , используя оценки леммы 1 , имеем

$$
\begin{aligned}
\|f(t)\|_{\mathbf{L}^{\infty}}= & \left\|\Delta u_{t}(t)\right\|_{\mathbf{L}^{\infty}} \leqslant\left\|\Delta \partial_{t} \mathscr{G}(t) u_{0}\right\|_{\mathbf{L}^{\infty}}+\left\|\Delta \mathscr{B}|u|^{\sigma} u(t)\right\|_{\mathbf{L}^{\infty}} \\
& +C \int_{\frac{t}{2}}^{t}\langle t-\tau\rangle^{-1}\left\|\Delta \mathscr{B}|u|^{\sigma} u(\tau)\right\|_{\mathbf{L}^{\infty}} d \tau \\
& +C \int_{0}^{\frac{t}{2}}\langle t-\tau\rangle^{-\frac{n}{2}-2}\left(\left\||u|^{\sigma} u(\tau)\right\|_{\mathbf{L}^{1}}+\left\|\mathscr{B}|u|^{\sigma} u(\tau)\right\|_{\mathbf{L}}\right) d \tau \\
\leqslant & C\langle t\rangle^{\left(1-\frac{n}{2} \sigma-\frac{n}{2}\right)(2 \sigma+1)}+C \int_{\frac{t}{2}}^{t}\langle t-\tau\rangle^{-1}\langle\tau\rangle^{\left(1-\frac{n}{2} \sigma-\frac{n}{2}\right)(2 \sigma+1)} d \tau \\
& +C \int_{0}^{\frac{t}{2}}\langle t-\tau\rangle^{-\frac{n}{2}-2}\langle\tau\rangle^{-\frac{n}{2} \sigma} d \tau \leqslant C\langle t\rangle^{-1-\frac{1}{\sigma}}
\end{aligned}
$$

для всех $t>0$, поскольку $\left(1-\frac{n}{2} \sigma-\frac{n}{2}\right)(2 \sigma+1) \leqslant-1-\frac{1}{\sigma}$ и $-\frac{n}{2}-1-\frac{n}{2} \sigma \leqslant-1-\frac{1}{\sigma}$, если $\sigma \in\left(0, \frac{2}{n}\right)$ достаточно близко к $\frac{2}{n}$. 
Как и выше, рассмотрим две вспомогательные задачи Коши (56) и (57) с достаточно малым $\varepsilon>0$. При этом задача (56) имеет достаточно малые начальные данные и малую правую часть $\varepsilon^{2}|f|$. Применяя принщип максимума к задаче $(56)$, получим точную оценку убывания по времени:

$$
\|U(t)\|_{\mathbf{L} \infty} \leqslant C\langle t\rangle^{-\frac{1}{\sigma}}
$$

откуда с помощью леммы 7 получим точную оценку убывания по времени для решения:

$$
\|u(t)\|_{\mathbf{L}^{\infty}} \leqslant C\langle t\rangle^{-\frac{1}{\sigma}}
$$

Оценим теперь $\mathbf{L}^{1, a}$-норму решения

$$
\begin{aligned}
\|u(t)\|_{\mathbf{L}^{1, a}} \leqslant & \left\|\mathscr{G}(t) u_{0}\right\|_{\mathbf{L}^{1, a}} \\
& +C \int_{0}^{t}\left(\left\|\mathscr{B}|u|^{\sigma} u(\tau)\right\|_{\mathbf{L}^{1, a}}+\langle t-\tau\rangle^{\frac{a}{2}}\left\|\mathscr{B}|u|^{\sigma} u(\tau)\right\|_{\mathbf{L}^{1}}\right) d \tau \\
\leqslant & C\langle t\rangle^{\frac{a}{2}}+C \int_{0}^{t}\langle\tau\rangle^{-1}\|u(\tau)\|_{\mathbf{L}^{1, a}} d \tau+C \int_{0}^{t}\langle t-\tau\rangle^{\frac{a}{2}}\langle\tau\rangle^{-1} d \tau \\
\leqslant & C\langle t\rangle^{\frac{a}{2}}+C \int_{0}^{t}\langle\tau\rangle^{-1}\|u(\tau)\|_{\mathbf{L}^{1, a}} d \tau,
\end{aligned}
$$

откуда с помощью леммы Гронуолла получаем оценку

$$
\|u(t)\|_{\mathbf{L}^{1, a}} \leqslant C\langle t\rangle^{\frac{a}{2}}
$$

для всех $t>0$. Аналогично оценим $\mathbf{L}^{1, a}$-норму правой части $f$. Как и при доказательстве теоремы 4, из леммы 1 находим

$$
\begin{aligned}
\|f(t)\|_{\mathbf{L}^{1, a}}= & \left\|\Delta u_{t}(t)\right\|_{\mathbf{L}^{1, a}} \leqslant\left\|\Delta \partial_{t} \mathscr{G}(t) u_{0}\right\|_{\mathbf{L}^{1, a}}+\left\|\Delta \mathscr{B}|u|^{\sigma} u(t)\right\|_{\mathbf{L}^{1, a}} \\
& +C \int_{0}^{\frac{t}{2}}\langle t-\tau\rangle^{-2}\left(\left\||u|^{\sigma} u(\tau)\right\|_{\mathbf{L}^{1, a}}+\langle t-\tau\rangle^{\frac{a}{2}}\left\||u|^{\sigma} u(\tau)\right\|_{\mathbf{L}^{1}}\right) d \tau \\
& +C \int_{\frac{t}{2}}^{t}\langle t-\tau\rangle^{-1}\left(\left\|\Delta \mathscr{B}|u|^{\sigma} u(\tau)\right\|_{\mathbf{L}^{1, a}}+\langle t-\tau\rangle^{\frac{a}{2}}\left\|\Delta \mathscr{B}|u|^{\sigma} u(\tau)\right\|_{\mathbf{L}^{1}}\right) d \tau \\
\leqslant & C\langle t\rangle^{\frac{a}{2}-1-\frac{1}{\sigma}+\frac{n}{2}}+C \int_{0}^{\frac{t}{2}}\left(\langle t-\tau\rangle^{-2}\langle\tau\rangle^{\frac{a}{2}-1}+\langle t-\tau\rangle^{\frac{a}{2}-2}\langle\tau\rangle^{-1}\right) d \tau \\
& +C \int_{\frac{t}{2}}^{t}\left(\langle t-\tau\rangle^{-1}\langle\tau\rangle^{\frac{a}{2}-1-\frac{1}{\sigma}+\frac{n}{2}}+\langle t-\tau\rangle^{\frac{a}{2}-1}\langle\tau\rangle^{-1-\frac{1}{\sigma}+\frac{n}{2}}\right) d \tau \\
\leqslant & C\langle t\rangle^{\frac{a}{2}-1-\frac{1}{\sigma}+\frac{n}{2}}
\end{aligned}
$$

поскольку $\sigma \in\left(0, \frac{2}{n}\right)$ достаточно близко к $\frac{2}{n}$. Таким образом, мы можем применить результаты работ [21], [22] и вычислить асимптотическое поведение решений $U(t, x)$ и $V(t, x)$ соответствуюших задач (56) и $(57)$. Отсюда с помошью леммы 7 
получаем точную оценку убывания по времени для решения с малым коэффициенTOM $\frac{2}{n}-\sigma$ :

$$
\|u(t)\|_{\mathbf{L}^{\infty}} \leqslant C\langle t\rangle^{-\frac{n}{2}}\left(1+C \varepsilon\left(\frac{2}{n}-\sigma\right)^{-\frac{1}{\sigma}} t^{\frac{1}{\sigma}-\frac{n}{2}}\right)^{-1}
$$

для всех $t>0$. Как и при доказательстве теоремы 4 , рассмотрим задачу Коши (68) для новых функций $(v(t, x), \varphi(t))$. Докажем оценку

$$
\|v(t)\|_{\mathbf{L}^{1, a}} \leqslant C\langle t\rangle^{\frac{a}{2}}
$$

для всех $t>0$. Из оценки (93) следует

$$
\|u(t)\|_{\mathbf{L}^{\infty}}^{\sigma} \leqslant C\langle t\rangle^{-\frac{n}{2} \sigma}\left(1+C \varepsilon^{\sigma}\left(\frac{2}{n}-\sigma\right)^{-1} t^{1-\frac{n}{2} \sigma}\right)^{-1} .
$$

В силу (92), (93), применяя лемму 1, получаем

$$
\begin{aligned}
& \left\|\int_{0}^{t} \mathscr{G}(t-\tau) \mathscr{B}\left(|u(\tau)|^{\sigma} v(\tau)-\frac{v(\tau)}{\theta} \int_{\mathbb{R}^{n}}|u(\tau)|^{\sigma} v(\tau) d x\right) d \tau\right\|_{\mathbf{L}^{1, a}} \\
& \leqslant C \int_{0}^{t}\left\||u(\tau)|^{\sigma} v(\tau)-\frac{v(\tau)}{\theta} \int_{\mathbb{R}^{n}}|u(\tau)|^{\sigma} v(\tau) d x\right\|_{\mathbf{L}^{1, a}} d \tau \\
& \leqslant C \varepsilon^{-\sigma}\left(\frac{2}{n}-\sigma\right) \int_{T}^{t}\langle\tau\rangle^{-1}\|v(\tau)\|_{\mathbf{L}^{1, a}} d \tau
\end{aligned}
$$

для всех $t>0$. Следовательно, из интегрального уравнения (61) имеем

$$
\begin{aligned}
\|v(t)\|_{\mathbf{L}^{1, a}} & \leqslant\|\mathscr{G}(t) v(0)\|_{\mathbf{L}^{1, a}}+C \varepsilon^{-\sigma}\left(\frac{2}{n}-\sigma\right) \int_{0}^{t}\langle\tau\rangle^{-1}\|v(\tau)\|_{\mathbf{L}^{1, a}} d \tau \\
& \leqslant C\langle t\rangle^{\frac{a}{2}}+\varepsilon \int_{0}^{t}\langle\tau\rangle^{-1}\|v(\tau)\|_{\mathbf{L}^{1, a}} d \tau
\end{aligned}
$$

для всех $t>0$, где $\varepsilon>0$ достаточно мало (напомним, что $\sigma<\frac{2}{n}$ является достаточно близким к $\frac{2}{n}$ ). Применяя лемму Гронуолла, получим оценку

$$
\|v(t)\|_{\mathbf{L}^{1, a}} \leqslant C\langle t\rangle^{\frac{a}{2}}
$$

для всех $t>0$. Аналогично, с помошью оценки (93) и леммы 1 получим

$$
\begin{aligned}
\|v(t)\|_{\mathbf{L}^{\infty} \leqslant} \leqslant & \|\mathscr{G}(t) v(0)\|_{\mathbf{L}^{\infty}}+C \| \int_{0}^{t} \mathscr{G}(t-\tau) \mathscr{B}\left(|u(\tau)|^{\sigma} v(\tau)\right. \\
& \left.-\frac{v(\tau)}{\theta} \int_{\mathbb{R}^{n}}|u(\tau)|^{\sigma} v(\tau) d x\right) d \tau \|_{\mathbf{L}^{\infty}} \\
\leqslant & C\langle t\rangle^{-\frac{n}{2}}+C \int_{0}^{\frac{t}{2}}(t-\tau)^{-\frac{n}{2}-\frac{a}{2}} \tau^{\frac{a}{2}-1} d \tau \\
& +C \varepsilon^{-\sigma}\left(\frac{2}{n}-\sigma\right) \int_{\frac{t}{2}}^{t}\langle\tau\rangle^{-1}\|v(\tau)\|_{\mathbf{L}^{\infty}} d \tau \\
\leqslant & C\langle t\rangle^{-\frac{n}{2}}+\varepsilon \int_{\frac{t}{2}}^{t}\langle\tau\rangle^{-1}\|v(\tau)\|_{\mathbf{L}^{\infty} d \tau}
\end{aligned}
$$


для всех $t>0$. Снова применяя лемму Гронуолла, приходим к оценке

$$
\|v(t)\|_{\mathbf{L}^{\infty}} \leqslant C\langle t\rangle^{-\frac{n}{2}}
$$

для всех $t>0$.

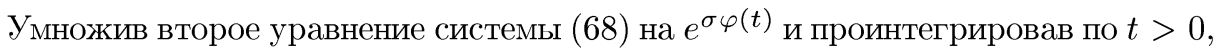
заменяя $e^{\sigma \varphi(t)}=h(t)$, получаем интегральное уравнение

$$
h(\tau)=1-\frac{\sigma \lambda}{\theta} \int_{0}^{\tau} \int_{\mathbb{R}^{n}}|v|^{\sigma} v d x d t
$$

Ввиду (94), используя лемму 8, видим, что

$$
\left|h_{m}(t)-h_{1}(t)\right| \leqslant \varepsilon h_{1}(t)
$$

для всех $t>0$, где

$$
\begin{gathered}
h_{1}(t)=1+\frac{2|\theta|^{\sigma} \eta}{2-n \sigma} t^{1-\frac{\sigma}{2} n}, \\
\eta=\sigma \lambda(4 \pi \alpha)^{-\frac{n}{2} \sigma}(1+\sigma)^{-\frac{n}{2}}
\end{gathered}
$$

Теперь асимптотические формулы устанавливаются точно так же, как и в конще доказательства теоремы 5. Теорема полностью доказана.

\section{Список литературы}

1. Amick C.J., Bona J.L., Schonbek M.E. Decay of solutions of some nonlinear wave equations // J. Diff. Eqs. 1989. V. 81. № 4. P. 1-49.

2. Bisognin $V$. On the asympototic behavior of the solutions of a nonlinear dispersive system of Benjamin-Bona-Mahony's type // Boll. Un. Mat. Ital. B. 1996. V. 10. № 7. P. 99-128.

3. Bona J. L., Luo L. Decay of solutions to nonlinear, dispersive wave equations // J. Diff. Integ. Eqs. 1993. V. 6. P. 961-980.

4. Bona J.L., Luo L. More results on the decay of solutions to nonlinear, dispersive wave equations // Discrete and Continuous Dynamical Systems. 1995. V. 1. P. 151-193.

5. Бесов О.В., Ильин В.П., Никольский С. М. Интегральные представления функций и теоремы вложения. М.: Наука, 1978.

6. Constantin A., Escher J. Wave breaking for nonlinear nonlocal shallow water equations // Acta Math. 1998. V. 181. № 2. P. 229-243.

7. Dix D. B. The dissipation of nonlinear dispersive waves: The case of asymptotically weak nonlinearity // Comm. P.D.E. 1992. V. 17. P. 1665-1693.

8. Ватсон Дж. Теория функций Бесселя. М.: Мир, 1944.

9. Габов С. А. Новые задачи математической теории волн. М.: Физматгиз, 1998.

10. Гаевский X., Грегер K., Захариас K. Нелинейные операторные методы и операторные дифференциальные уравнения. М.: Мир, 1978.

11. Галактионов В.А., Курдюмов С.П., Самарский А. А. Асимптотические собственные функции задачи Коши для нелинейных параболических уравнений // Матем. сб. 1985. T. 126. № 4. C. 435-472.

12. Демиденко Г. В., Успенский С. В. Уравнения и системы, не разрешенные относительно старшей производной. Новосибирск: Научная книга, 1998. 
13. Доброхотов C. Ю. Нелокальные аналоги нелинейного уравнения Буссинеска для поверхностных волн над неровным дном и их асимптотические решения // ДАН СССР. 1987. Т. 292. № 1. С. 63-67.

14. Езоров И.Е., Пятков С.Г., Попов С.В. Неклассические дифференциально-операторные уравнения. Новосибирск: Наука, 2000.

15. Escobedo M., Kavian O. Asymptotic behavior of positive solutions of a non-linear heat equation // Houston J. of Math. 1987. V. 13. № 4. P. 39-50.

16. Escobedo M., Kavian O., Matano $H$. Large time behavior of solutions of a dissipative nonlinear heat equation // Comm. Partial Diff. Eqs. 1994. V. 20. P. 1427-1452.

17. Favini A., Yagi A. Degenerate differential equations in Banach spaces. N. Y.: Marcel Dekker, Inc, 1999.

18. Fujita $H$. On blowing-up of solutions of the Cauchy problem for $u_{t}=\Delta u+u^{1+\alpha} / / \mathrm{J}$. Fac. Sci. Univ. of Tokyo. Sect. I. 1966. V. 13. P. 109-124.

19. Gmira A., Veron L. Large time behavior of solutions of a semilinear parabolic equation in $\mathbb{R}^{N}$ // J. Diff. Eqs. 1984. V. 53. P. 258-276.

20. Hayashi N., Kaikina E. I., Naumkin P. I. Large time behavior of solutions to dissipative nonlinear Schrödinger equation // Proceedings of Royal Soc. Edingburgh. 2000. V. 130A. P. 1029-1043.

21. Hayashi N., Kaikina E.I., Naumkin P.I. Global existence and decay in time of small solutions to Landau-Ginzburg type equations // J. d'Analyse Mathematique. 2003. V. 90. P. 141-173.

22. Hayashi N., Ito N., Kaikina E. I., Naumkin P. I. On some nonlinear dissipative equations with sub-critical nonlinearities // Taiwanese J. of Math. 2004. V. 8. P. 135-154.

23. Hayashi N., Naumkin P.I. Asymptotics for Korteweg-de Vries-Burgers equation // J.M.M.A. (в печати).

24. Кайкина Е.И., Наумкин П.И., Шишмарёв И. А. Асимптотика решений нелинейных диссипативных систем уравнений // Изв. РАН. Сер. матем. 2004. Т. 68. № 3. С. 29-62.

25. Kamin S., Peletier L. A. Large time behaviour of solutions of the heat equation with absorption // Ann. Scuola Norm. Sup. Pisa. 1985. V. 12. P. 393-408.

26. Karch $G$. Asymptotic behaviour of solutions to some pseudoparabolic equations // Math. Methods in the Applied Sciences. 1997. V. 20. P. 271-289.

27. Karch $G$. Large-time behavior of solutions to nonlinear wave equations: higher-order asymptotics // Math. Methods in the Applied Sciences. 1999. V. 22. P. 1671-1697.

28. Kavian $O$. Remarks on the large time behavior of a nonlinear diffusion equation // Ann. Inst. Henri Poincaré. Analyse non linéaire. 1987. V. 4. № 5. P. 423-452.

29. Кожсанов А.И. Параболические уравнения с нелинейным нелокальным источником // Сиб. матем. журн. 1994. Т. 35. № 5. С. 1062-1073.

30. Кохсанов А.И. Начально-краевая задача для уравнения типа обобщенного уравнения Буссинеска с нелинейным источником // Матем. заметки. 1999. Т. 65. № 1. С. 70-75.

31. Корпусов М. О., Плетнер Ю. Д., Свешников А. Г. О нестационарных волнах в средах с анизотропной дисперсией // ЖВМиМФ. 1999. Т. 39. №6. С. 968-984.

32. Корпусов M. О., Свешников А.Г. Трехмерные нелинейные эволюционные уравнения псевдопараболического типа в задачах математической физики // ЖКВМиМФ. 2003. T. 43. № 12. C. 1835-1869.

33. Levine H. A. Some nonexistence and instability theorems for solutions of the formally parabolic equations of the form $P u_{t}=-A u+F(u) / /$ Arch. Rational Mech. Anal. 1973. V. 51. P. 371-386.

34. Mei $M$. $L_{q}$-decay rates of solutions for Benjamin-Bona-Mahony-Burgers equations // J. Diff. Eqs. 1999. V. 158. P. 314-340.

35. Mei M., Schmeiser C. Asymptotic profiles of solutions for the BBM-Burgers equations // Funkcial. Ekvac. 2001. V. 44. P. 151-170.

36. Митидиери Э., Похожаев С. И. Априорные оценки и отсутствие решений дифференциальных неравенств в частных производных // Тр. МИАН. 2001. Т. 232. С. 1-222. 
37. Naumkin P. I., Shishmarev I. A. Nonlinear Nonlocal Equations in the Theory of Waves. V. 133. Providence: AMS, 1994.

38. Prado R., Zuazua E. Asymptotic expansion for the generalized Benjamin-Bona-Mahony-Burgers equation // Diff. Integ. Eqs. 2002. V. 15. № 12. P. 1409-1434.

39. Самарский А.А., Галактионов В.А., Курдюмов С.П., Михайлов А.П. Режимы с обострением в задачах для квазилинейных параболических уравнений. М.: Наука, 1999.

40. Свиридюк Г. А., Федоров В.Е. Аналитические полугруппы с ядрами и линейные уравнения типа Соболева // Сиб. матем. журн. 1995. Т. 36. № 5. С. 1130-1145.

41. Соболев С. Л. Об одной новой задаче математической физики // Изв. АН СССР. Сер. матем. 1954. Т. 18. №1. С. 3-50.

42. Стейн Е. Сингулярные интегралы и дифференциальные свойства функций. М.: Мир, 1970.

43. Stefanelli $U$. On a class of doubly nonlinear nonlocal evolution equations // Diff. Integ. Eqs. 2002. V. 15. № 8. P. 897-922.

44. Титчмари Е. С. Введение в теорию интегралов Фурье. М.: Мир, 1944.

45. Уизем Дж. Б. Линейные и нелинейные волны. М.: Мир, 1977.

46. Шишмарёв И. А. Об одном нелинейном уравнении типа Соболева // Дифф. уравн. 2005. T. 41. № 1.

47. Schonbek M. E. The Fourier splitting method // Advances in geometric analysis and continuum mechanics. Cambridge: Internat. Press, 1995. P. 269-274.

48. Showalter R.E. Monotone operators in Banach space and nonlinear partial differential equations. Providence: AMS, 1997.

49. Zhang L. Decay of solutions of generalized Benjamin-Bona-Mahony equations // Acta Math. Sinica. New Series. 1994. V. 10. P. 428-438.

50. Zhang L. Decay of solutions of generalized Benjamin-Bona-Mahony-Burgers equations in $n$-space dimensions // Nonlinear Analisis T.M. A. 1995. V. 25. P. 1343-1369.

51. Zuazua E. A dynamical system approach to self similar large time behavior in scalar convection-diffusion equation // J. Diff. Eqs. 1994. V. 108. P. 1-35.

МГУ им. М.В. Ломоносова

Поступило в редакцию

E-mail: shish@voxnet.ru, 30.09 .2004

pavelni@matmor. unam.mx 